\title{
On the applicability of models for outdoor sound (A)
}

\section{Rasmussen, Karsten Bo}

\section{Published in:}

Acoustical Society of America. Journal

Link to article, DOI:

$10.1121 / 1.425063$

Publication date:

1999

\section{Document Version}

Publisher's PDF, also known as Version of record

Link back to DTU Orbit

Citation (APA):

Rasmussen, K. B. (1999). On the applicability of models for outdoor sound (A). Acoustical Society of America. Journal, 105(2), 1064-1065. https://doi.org/10.1121/1.425063

\section{General rights}

Copyright and moral rights for the publications made accessible in the public portal are retained by the authors and/or other copyright owners and it is a condition of accessing publications that users recognise and abide by the legal requirements associated with these rights.

- Users may download and print one copy of any publication from the public portal for the purpose of private study or research.

- You may not further distribute the material or use it for any profit-making activity or commercial gain

- You may freely distribute the URL identifying the publication in the public portal

If you believe that this document breaches copyright please contact us providing details, and we will remove access to the work immediately and investigate your claim 


\title{
Posters from various technical sessions remain on display in the Poster Gallery.
}

Posters from sessions which contain both lecture and poster presentations will be attended by the authors as listed below.

$\begin{array}{ll}\text { 1pNSa12 } & \text { Mirowska, Marianna } \\ \text { 2aPAb13 } & \text { Robsman, Vadim A. } \\ & \text { Robsman, Vadim A. } \\ \text { 2aPAb14 } & \text { Hutchins, D. A. } \\ \text { 2aPAb15 } & \text { Murphy, William J. } \\ \text { 3aNSb12 } & \text { Zannin, Paulo H. T. } \\ \text { 3aNSb13 } & \end{array}$

$$
\text { 2:00-4:00 }
$$

Assessment of low frequency noise in dwellings. New Polish recommendations

Nonlinear acoustics testing of defect for evaluation of risk of insurance of the therman electric power stations

Experience in nonlinear wave testing of constructions inside the earthquake area Experiments in solids using air-coupled ultrasound

Four protectors in search of a rating system

Effects of cup, cushion, headband force, and foam lining on the attenuation of earmuffs

Also, the following poster sessions are scheduled:

Poster Session $2 \mathrm{pSCb}$

Poster Session 2pSPb

TUESDAY AFTERNOON, 16 MARCH 1999

ROOM H105, 1:55 TO 4:00 P.M.

\author{
Session 2pAAa \\ Architectural Acoustics: Worship and Theatre Spaces \\ Emmanuel G. Tzekakis, Cochair \\ Department of Architecture, Aristotle University of Thessaloniki, 540 06, Thessaloniki, Greece \\ Daniel E. Commins, Cochair \\ Commins Acoustics Workshop, 15 rue Laurence Savart, F-75020 Paris, France
}

Chair's Introduction-1:55

Contributed Papers

2:00

2pAAa1. The acoustical design of a new open air theater in Thessaloniki, Greece. Emmanuel G. Tzekakis (Dept. of Architecture, Aristotle Univ. of Thessaloniki, 540 06, Thessaloniki, Greece) and Gottfried Schubert (Consultant in Acoust., Athens, Greece, emil49@compulink.gr)

The international experience in the design of open air theaters is limited. Most new theaters are used exclusively with electroacoustic installations, because they are very large or in very noisy surroundings. As a result, no new architectural or acoustical breakthroughs are available. The new theater reported in this paper is a 5000 seat open air theater, with a shape derived from the ancient Greek prototypes, optimized for best listening conditions without electroacoustic installations. The background noise level reduction and the geometry of the theater were the main concerns of the acoustical design. Modeling tools were used to predict the final acoustical conditions. Measurements made in the almost ready theater indicate that using known and reliable prototypes in a careful way results in high quality acoustical conditions.
2:20

2pAAa2. The ancient Greek theater and its acoustical quality for contemporary performances. Gottfried Schubert (Consultant in Acoust., 22 Apolonos Str., 153 44, Kantza Pallini, Athens, Greece, emil49@compulink.gr) and Emmanuel G. Tzekakis (Aristotle Univ. of Thessaloniki, 540 06, Thessaloniki, Greece)

The acoustical quality of the ancient Greek theater is often described as a phenomenon with mythical dimensions. According to recent experiences with contemporary theater performances at famous historical ancient Greek theaters, the acoustical situation is much more simple and even more problematic than described. To understand the real situation, it is helpful to review the evolution of the ancient theater in terms of construction as well as the theater play as a religious and cultural event. The Dionysus Theater of Athens is one of the most important examples to base this review on. Modern theater festivals also take place in the ancient theater of Epidaurus, which compared to many others, is in a remarkably good condition especially after its restoration. Nevertheless, the scene building is missing and this leads to several acoustical problems. The Epidaurus Theatre is used as an example for the description of the experiences with contemporary performances in ancient Greek theaters. 
2pAAa3. Acoustic measurement of Shakespeare's Globe Theatre, London. Russell Richardson and Bridget M. Shield (South Bank Univ., 103 Borough Rd., London SE1 0AA, UK, richarjr@sbu.ac.uk)

In the late 1960's, Sam Wanamaker initiated the reconstruction in London of the theatre built for William Shakespeare's company of players in the late 16th century. The theatre was built in the traditional manner to be as faithful to the original as surviving documentation allows, and has a circular auditorium with an open roof. The theatre was opened for performances in the summer of 1997. An acoustic survey of the theatre has been carried out. Measurements have been made of reverberation time (RT), early decay time (EDT), clarity index (C50), definition (D50), and rapid speech transmission index (RASTI) within the theatre's auditorium. Various source positions were chosen on the stage after consultation with voice coaches and actors at the theatre, and receiver positions were chosen to provide uniform coverage of all the major audience areas. The results of these measurements indicate that, despite the theatre's open-air construction, the amount of early energy provided to most audience areas results in reasonable levels of speech intelligibility, although achieving an adequate speech sound level is more of a problem. This is clearly exacerbated by the theatre's open-air design, and its position in central London under the main flight path to London's Heathrow Airport. The reverberant characteristics of the auditorium are generally within acceptable limits for modern theatres.

\section{3:00}

2pAAa4. Acoustical evaluation of auditoria in Taiwan built before 1975. Weihwa Chiang and Zenjang Juang (Taiwan Univ. of Sci. and Tech., 43, Section 4, Keelung Rd., Taipei City 106, Taiwan, WHCH@mail.ntust.edu.tw)

Investigations were conducted regarding the acoustical characteristics of existing auditoria built before 1975. Discussions of the historical evolution since Japanese occupation were followed by architectural and acoustical analysis of 21 cases. Although many auditoria built during Japanese occupation were not designed for musical performances, they are more reverberant but smaller than the ones built after World War II. However, $62 \%$ of the selected cases were constructed with windows with $4 \mathrm{~mm}$ or thinner glazing on sidewalls or around the upper perimeter. Analysis of two auditoria were made regarding the performances of room acoustics, background noise control, and sound system. The room acoustics in both auditoria are suitable for chamber music but are too dry and too loud for orchestra music. Average background noises reach NC40 with AC off and NC50 with AC on. Both sound systems could not give correct directions of sound images, although they provide acceptable output level (96 dB SPL) and speech intangibility (STI 0.57) in the $2000-\mathrm{Hz}$ band. With growing concerns about energy preservation in Taiwan, the current study could provide useful information for renovating or remodeling an existing auditorium. [Work supported by the National Science Foundation of Taiwan.]
2pAAa5. Restoration by means of sound reinforcement of a church of the Renaissance. Jose Romero-Faus, Alicia Gimenez-Perez, Albert Marin-Sanchis, and Antonio Sanchis-Sabater (Appl. Phys. Dept., High Tech. School of Industrial Eng., Polithechnic Univ. of Valencia, P.O. Box 22012, 46080 Valencia, Spain, agimenez@ fis.upv.es)

This work summarizes the project of the acoustic restoration of a Church of the Renaissance, XV Century, after the architectural restoration carried out in it throughout the last 10 years, with volume, $9000 \mathrm{~m}^{3}$; total surface, $3000 \mathrm{~m}^{2}$; room constant, $R=1110 \mathrm{~m}^{2}$; reverberation time to $500 \mathrm{~Hz}, 1.8 \mathrm{~s}$; inteligibility from the altar to the last banks, 0.34 ; loss of sound level, $7 \mathrm{~dB}$. Since the acoustic response for the music and the song was good, the one which should improve above all was the intelligibility. Specifically, this project was undertaken to provide a uniform sound coverage to all the hearing zone, an adequate sound level, the nonexistence of perceptible late reflections, and the adequate intelligibility characteristic of the room. Bearing in mind, furthermore, that the acoustics of the enclosure could not be improved by means of acoustic materials, the adopted solution was to use an electroacoustic system, putting a centralized cluster of loudspeakers over the altar, to $8 \mathrm{~m}$ of height, that assured the sufficient sound reinforcement. This solution improved the intelligibility, which became 0.53 , as well as the coverage field. These results have been validated by means of a ray tracing program.

\section{Contributed Posters}

These posters will be on display in the Poster Gallery from Monday to Wednesday, 15-17 March. Authors will be at their posters from 10:00 a.m. to 12:00 noon on Wednesday, 17 March.

\begin{abstract}
2pAAa7. Room acoustical measurements and architectural documentation of auditoriums in Berlin considering the speech intelligibility in dependence of spatial distribution of sound fields. Peter S. Krämer (GEBRA Gesellschaft für Bau- und Raumakustik mbH, Agricolastr. 26, D-10555 Berlin, Germany)
\end{abstract}

Measurements of spatial room acoustical impulse responses were performed to provide a database for the examination of speech intelligibility (SI) in real rooms. As is well known, the spatial distribution of reflections can influence the SI, especially in reverberant rooms. Nevertheless, the qualitative and quantitative dependences are mostly unknown. Room acoustical measurements thus were realized using a dodecahedron loud- speaker for measuring reverberation time, an interference-free loudspeaker with omnidirectional characteristic for recording impulse responses, and an artificial speaker and a dummy head microphone for measurements of SI criteria and recording of logatom syllables. A specially constructed spaced four-capsule microphone in tetrahedron arrangement was used for measuring also. The four-microphone analysis technique can recognize intensity and direction of the early reflections [Odjija and Krämer (1999)]. Results of the measurements will be documented and published on a CDROM, together with the architectural data of the auditoriums. First results of the room acoustical measurements are presented. Prior studies have shown that reproducing the measured binaural cues with headphones does 
not influence the SI significntly. Subjective measurements of SI and analysis of spatial dependences will be performed in a second stage of the project.

2pAAa8. Simulated acoustic field in mudejar-gothic churches. Miguel Galindo, Teofilo Zamarreno, Sara Giron (Dept. Fisica Aplicada, Universidad de Sevilla, Spain, teofilo@ cica.es), Juan J. Sendra, and Jaime Navarro (Dept. Construcciones Arquitectonicas, Universidad de Sevilla, Spain)

The most significant acoustic parameters in a set of mudejar-gothic churches were measured (all the churches were located in the city of Seville). From the analysis of these data important conclusions were otained about the behavior of the reverberant field versus source-receiver distance [J. Sendra et al., Computational Acoustics and its Environmental
Applications-II (Computation on Mechanics Publications, Southampton, 1997), pp. 139-148] from Barron's revised theory [Barron et al., J. Acoust. Soc. Am. 84, 618-628 (1988)] and the relations between several acoustics quality descriptors: C80, D50, and RASTI [Zamarreno et al., Proceedings Acustica-98, Lisbon (1998)]. The three most representative churches were chosen to carry out a computer simulation with the RAYNOISE software. Absorption coefficient values used in the model have been adjusted using measured reverberation time data, and thus the results were obtained for the other acoustic parameters in the different octave bands. It was proven that, in general, simulated values are coherent with the experimental results. Moreover, according to other authors [L. Marshall, J. Acoust. Soc. Am. 94, 2251-2261 (1994)], it has also been proven that C80 and D50 indices need no spectral analysis and their broadband values and their averages in significant bands $(500-2000 \mathrm{~Hz})$ are indifferent.

TUESDAY AFTERNOON, 16 MARCH 1999

ROOM H105, 4:15 TO 6:20 P.M.

\author{
Session 2pAAb \\ Architectural Acoustics: Perceptual Aspects in Rooms \\ Hideki Tachibana, Cochair \\ Institute of Industrial Science, Tokyo University, Roppongi 7-22-1, Minato-ku, Tokyo 106-8558, Japan \\ Roberto Pompoli, Cochair \\ Engineering Department, University of Ferrara, Via G. Saragat 1, 44100 Ferrara, Italy \\ Chair's Introduction-4:15 \\ Invited Paper \\ 4:20
}

2pAAb1. An evaluation of scattered reflections in sound fields. Y. Suzumura, Y. Ando (Grad. School of Sci. and Technol., Kobe Univ. Rokkodai, Nada, Kobe 657-8501 Japan, 966d852n@kobe-u.ac.jp), M. Oowaki (Kumagai-Gumi Tech. Res. Labs., Tsukuba, Japan), Toshiki Iizuka, and I. Yamamoto (Arch. \& Env. Res. Ltd., Kobe, Japan)

\begin{abstract}
Although a large number of studies have been made on a theory of scattering, no studies have been attempted to evaluate the scattered sound fields in a concert hall. This paper will show an evaluation method of sound fields that involve scattered reflection by a circular-columns array installed in front of both side walls. Measurements were performed by use of a 1/10 scale model of the concert hall. Here, scattered sound fields are evaluated by four acoustic factors (SPL, DT1, Tsub; IACC, and in addition WIACC and tauIACC). It is found that the IACC and Dt1 of the sound fields of the concert hall are improved with use of the circular columns array.
\end{abstract}

\title{
Contributed Papers
}

\section{4:40}

2pAAb2. Listener perception of spatial impression and auditory source width and their influence on overall impression in real rooms and headphone studies. Martin A. Gold (Dept. of Architecture, Univ. of Florida, Gainesville, FL 32611-5702)

Comparisons of listener evaluations of music in real room situations and in headphone listening studies were used to assess the contribution of reverberance, loudness, auditory source width (ASW), and spatial impression on the overall impression of music listening and to evaluate their role as independent acoustic variables. Room studies were conducted using large numbers of average listeners at designated locations with a live sound source. Headphone listening studies were conducted using anechoic recordings with simulated acoustic environments (early reflections and reverberation) having different configurations of early and late sound energy. Directional components of the early and late energy were independently modeled as part of the experiment (Gold, 1994). Regression models were conducted to assess the interrelationship of independent variables on listener overall impression. In many cases, independent elements could be identified as contributing to overall impression; however, these elements were not the same in each case. For example, in cases where reverberance is not present, loudness and ASW become greater contributors. In addition, an attempt was made to find out if there is a listener distinction between ASW (a widening of the source sound) and spatial impression (the quality of being immersed in the sound) 
ity. The influence of the stage visual distance on the auditory apparent

2pAAb3. Controls of a modulated delay time of reflection enhancing subjective preference. Junko Atagi, Yoichi Ando (Grad. School of Sci. and Technol., Kobe Univ., Rokkodai, Nada, Kobe, 657-8501 Japan, 968d803n@kobe-u.ac.jp), and Yasutaka Ueda (Hazama Corp., Karima, Tsukuba, 305-0822 Japan)

Previously, the most preferred delay time of reflection for timeinvariant sound fields corresponds to the effective duration of the autocorrelation function $\left(\tau_{e}\right)$ of source signals [Ando, Concert Hall Acoustics (Springer-Verlag, New York, 1985)]. However, the SPL fluctuation in a real room is fluctuated and the model of such a time-variant system is proposed by the direct sound and the varying delay time of reflection [Ueda and Ando, J. Acoust. Soc. Am. 102, 2771-2775 (1997)]. To realize more excellent sound fields than time-invariant sound fields, a method of controlling a modulation of the delay time of reflection is discussed. Experiments are performed on the modulation methods to enhance subjective preference in a sound field. The following methods are examined to control the delay time of reflection in the experiment: (1) sinusoidally modulated delay time, (2) random modulated delay time, (3) delay time corresponding to the change of the running $\tau_{e}$ of the source signal, and (4) fixed delay time of reflection at the minimum value of the running $\tau_{e} \mathrm{~s}$.

\section{$5: 20$}

2pAAb4. Effect of closely spaced high-frequency reflections on perception of music. Paul T. Calamia and Scott D. Pfeiffer (Kirkegaard \& Assoc., 4910 Main St., Downers Grove, IL 60515)

Experience in three concert halls with complaints about harsh highfrequency response led to the investigation of acoustic effects in these halls. The use of wall treatments intended to be acoustically diffusive or transparent, or intended to make a fan-plan more similar to a shoe-box hall, resulted in repetitive, closely spaced, high-frequency reflections. Effects have been measured in place and in laboratory simulations. Examples of the wall treatments and corresponding measurements will be discussed, as will recommendations for achieving a more desirable frequency response.

\section{5:40}

2pAAb5. Influence of sensory interactions between vision and audition on the perceptual characterization of room acoustics. Chrysanthy Nathanail (Laboratoire d'Acoustique Musicale, Université Paris VI, Tour 66, Case 161, 4, place Jussieu, 75252, Paris Cedex 05, France), Catherine Lavandier (Université Cergy-Pontoise), Jean-Dominique Polack (Université Paris VI, France), and Olivier Warusfel (Ircam, Paris 75004, France)

Broad evidence on sensory interactions between vision and audition suggests that visual information available to listeners-spectators in concert halls is likely to interfere in the evaluation process of the acoustical qual- distance was studied in four magnitude estimation paradigms. Sound stimuli providing successive impressions of auditory distance were generated by a virtual room acoustics processor ("Spatialisateur") in binaural format and presented separately or coupled with 3-D concert hall pictures. Subjects were asked to judge the auditory distance. Pilot experiments I and II obtain a first qualitative observation of the effects and study a progressive change observed at the responses. Main experiments III and IV adopt two different procedures of stimuli presentation and improved sound reproduction and attempt a first objective evaluation of the results. The analysis of variance performed on the responses reveals a small offset-type influence of the visual stimuli on the auditory distance: i.e., the same auditory stimuli appear more distant when presented with distant stage views. In addition, differences between unimodal (auditory stimuli alone) and bimodal tests suggest that vision enhances the "depth" of the auditory field and provides a spatial frame of reference for auditory judgments.

\section{6:00}

2pAAb6. Perception of sweep sounds caused by reflective pulse train in a rectangular reverberation room. Kenji Kiyohara, Ken'ichi Furuya, Masashi Tanaka, and Yutaka Kaneda (NTT Human Interface Labs., 3-9-11, Midori-cho, Musashino-shi, Tokyo, 180-8585 Japan)

Elevated frequency sweep sounds were perceived when a pulsive sound (a handclap) was generated in a rectangular reverberation room. The mechanism of this phenomenon was investigated. First, reflection of the pulsive sound (a pulse train) was simulated using the image method. The sweep sounds were also perceived in the simulated pulse train. It was assumed that the pulse train, whose intervals decreased inversely in proportion to time, caused the sweep sound sensation. The existence of such a pulse train was confirmed in the simulated pulse train. Then, this phenomenon was investigated theoretically based on algebraic number theory. A pulse train whose intervals decrease inversely proportional to time becomes a pulse train of equal intervals when it is represented on the power of time $\left(t^{2}\right)$ axis. On the other hand, considering a cubic room for simplicity, reflective pulse sounds arrive at $\left(l^{2}+m^{2}+n^{2}\right) L^{2} / c^{2}$ on the $t^{2}$ axis $(l, m, n$ : positive integer, $L$ : room dimension, $c$ : sound velocity). The number theory proves that $\left(l^{2}+m^{2}+n^{2}\right)$ covers almost all integers. In other words, reflective pulsive sounds arrive with equal time intervals of $L^{2} / c^{2}$ on the $t^{2}$ axis. This algebraic regularity causes a listener to experience the sweep sound sensation.

\section{Contributed Posters}

These posters will be on display in the Poster Gallery from Monday through Wednesday, 15-17 March. Authors will be at their posters from 10:00 a.m. to 12:00 noon on Wednesday, 17 March.

2pAAb7. Appropriate bandwidth and integration time for measurements of the degree of interaural cross correlation as a measure of apparent source width in concert halls. Masayuki Morimoto (Environ. Acoust. Lab., Faculty of Eng., Kobe Univ., Nada, Kobe, 657-8501 Japan, mrmt@kobe-u.ac.jp) and Kazuhiro Iida (Matsushita Comm. Ind. Co., Ltd., Saedo, Tsuzuki, Yokohama, 224-8539 Japan)

ISO 3382 Annex suggests using the degree of interaural cross correlation (ICC) as a measure of apparent source width (ASW), while mentioning that the choice of bandwidth for test signals and the integration time are uncertain. Needless to say, what is important for a physical measure is that it is well correlated with the subjective effect, in this case ASW. This paper describes psychological experiments to investigate the appropriate bandwidth for test signals and the integration time for measuring ICC to evaluate ASW. The experimental results indicate that (1) measurements of ICC with 1/3-oct bands are preferred for evaluating ASW, whereas the use of wide band and 1/1-oct band signals as described in the ISO standard are not, and (2) measurement of ICC for all reflections including both the early reflections and later reverberant sound is most suitable for evaluating ASW, rather than the early reflections alone. 
2pAAb8. The interrelation between the music sound clarity index $C_{80}$ and the subjective evaluation of the hall acoustics. Vytautas $\mathrm{J}$. Stauskis (Vilnius Gediminas Tech. Univ., Sauletekio al. 11, LT 2040, Vilnius, Lithuania)

It has been established by acoustic investigations in actual halls that the clarity index $\mathrm{C}_{80}$ may have optimum recommended values, while subjectively the sounding of music in those halls may be evaluated as poor. Three halls were selected for investigations: (1) a church with the volume of $27000 \mathrm{~m}^{3}$, (2) Vilnius Opera Theatre with the volume of $8700 \mathrm{~m}^{3}$, and (c) a concert hall with the volume of $4900 \mathrm{~m}^{3}$. In hall (1), the values of $\mathrm{C}_{80}$ are from -1.4 to $-3.9 \mathrm{~dB}$. The reverberation time is $6.6 \mathrm{~s}$. According to subjective evaluations, the acoustics of this hall is suitable neither for classical nor romantic music. In hall (2), the values of $\mathrm{C}_{80}$ vary from -1.1 to $2.4 \mathrm{~dB}$ in all rows in the stalls. The reverberation time is very short and varies between $1.2 \mathrm{~s}$. The sound of an orchestra in the hall is evaluated as very poor. In hall (3), the values of $\mathrm{C}_{80}$ are within the range of -1.0 to $2.8 \mathrm{~dB}$ and the reverberation time is $1.55 \mathrm{~s}$. The sound of chamber music is evaluated as good, while that of symphony music is evaluated as satisfactory. This shows that this subjective indicator may not provide full characterization of the hall acoustics.

\title{
Session 2pAB
}

\section{Animal Bioacoustics: Physics of Animal Bioacoustics II}

\author{
Whitlow W. L. Au, Cochair \\ Hawaii Institute of Marine Biology, P.O. Box 1106, Kailua, Hawaii 96734, USA \\ Roland Aubauer, Cochair \\ Institute of Zoology II, University of Erlangen, Staudtstrasse 5, D-91058 Erlangen, Germany \\ Invited Paper
}

2:00

\begin{abstract}
2pAB1. Biophysics of peripheral auditory systems in three teleost fish. Mardi C. Hastings (Dept. of Mech. Eng., Ohio State Univ., 206 W. 18th Ave., Columbus, OH 43210)

Many types of auditory systems are found in the more than 20000 different species of teleost fish. These unique sensors may detect extremely small acoustic particle velocities as well as acoustic pressure. Auditory bandwidths may range from very low frequencies to ultrasonic regimes depending on the species. Several experimental studies conducted over the last 15 years have yielded data which indicate that auditory sensitivity, bandwidth, and source localization are primarily controlled by the biomechanics of the periphery. In these studies in vivo displacements of peripheral auditory organs were measured noninvasively in response to tones emitted from an underwater speaker. The measurements were then correlated with mathematical models formulated from the basic physics of each system. Results are presented for three different species, goldfish (Carassius auratus), oscars (Astronotus ocellatus), and shad (Alosa sapidissima), and compared with auditory capabilities determined in behavioral and physiological studies reported in the literature.
\end{abstract}

\section{Contributed Papers}

\section{2:20}

2pAB2. Development of a software package calculating zones of influence on marine mammals around industrial noise sources. Christine Erbe and David M. Farmer (IOS-Ocean Acoust., 9860 W. Saanich Rd., Sidney, BC V8L 4B2, Canada, erbec@dfo-mpo.gc.ca)

A software package is presented which estimates zones of interference around underwater noise sources affecting marine mammals. An ocean sound propagation model based on ray theory computes the spreading of complex underwater sound such as broadband animal vocalizations and man-made noise. On a grid of receiver locations (representing the affected marine mammal), the received signal and noise sound spectra are compared. Given a species-specific audiogram, the software package plots zones of audibility around the noise source. Given species-specific vocalizations, zones of masking are plotted based on results obtained during an earlier study which measured masked hearing thresholds of a beluga vocalization in icebreaker noise with a trained beluga whale. Tools developed during this study (such as an artificial neural network and critical band methods) are incorporated in the software package and can be used to predict zones of masking for industrial noise other than the types directly measured with the beluga. Zones of discomfort, injury, and hearing loss could be plotted if thresholds were known or using current estimates. The software package is applicable to a variety of ocean environments requiring location-specific physical oceanography input data.

\section{2:40}

2pAB3. Whistle signals of sturgeons and cetaceans. Larissa $\mathrm{K}$. Tolstoganova (17, V. Krasnoselskaya, Moscow 107140, Russia, tesler@vniro.ecosrv.fipc.ru)

There are a lot of recordings of acoustic signals emitted by cetaceans and a few studies on sturgeon sounds. The signal analysis showed that all heard species of sturgeons and likewise cetaceans produce whistles and impulse signals consisting of broadband pulses. Sturgeon whistles may be separated into tone whistle with line spectrum, narrow-band whistle with narrow-band spectrum, FM whistle with narrow-band or broadband summary spectrum, and line or narrow-band instantaneous spectra. Acipenser stellatus, for example, emit tone and narrow-band signals consisting of one to seven components. The first component frequency is 2 to $3.5 \mathrm{kHz}$. The highest one is up to $16 \mathrm{kHz}$. Acipenser baeri and Acipenser guldenstadti emit all signal types. The frequency spectrum is up to $18 \mathrm{kHz}$. More often Acipenser Baeri produce FM whistles having one to five components 
which frequency varies directly as the square or cube of time. The minimal frequency of the first component is $1.0-3.5 \mathrm{kHz}$. The frequency range of the component is up to $2.5 \mathrm{kHz}$. Acipenser guldenstadti more often produce FM whistles having one to three components, which frequency varies inversely as the square root of time. The frequency range of the component is up to $7 \mathrm{kHz}$.

\section{3:00}

2pAB4. Validation of a system for synthesizing distant signatures of underwater explosions for sea mammal hearing studies. Joseph A. Clark (CDNSWC, Code 734, Bethesda, MD 20084 and COMB, Ste. 236, Columbus Ctr., 701 E. Pratt St., Baltimore, MD 21202), Jane A. Young (CDNSWC, Bethesda, MD 20084 and COMB, Baltimore, MD 21202), and Joel B. Gaspin (IHNSWC, Indian Head, MD 20640)

A system for synthesizing distant signatures of underwater explosions was described in an earlier talk [J. A. Clark and Jane A. Young, J. Acoust. Soc. Am. 102, 3177(A) (1997)]. The system is now being used to study expected temporary threshold shifts (TTS) of sea mammals near underwater explosive test ranges in the Baltic sea. The results of these studies will be used to define safe range criteria for mammal hearing which will then be incorporated into the methodology for assessing potential damage to marine mammal hearing. Typical underwater explosion signals (time histories) were selected from an extensive set of blast signals computed for a variety of charge weights, explosion depths, ranges from the blast, and water conditions specified by sound-speed profiles and water depths. The selected signals were synthesized and measured at the location of the test mammals (bottlenose dolphins). The fidelity of the synthesized signals to the computed waveforms was determined by comparing such characteristics as peak pressures, positions of major spikes in the time histories, and one-third octave band energy spectra. Examples of typical signals used for the TTS studies and their measured characteristics will be presented.

\section{$3: 20$}

2pAB5. Information entropy of humpback whale song. Ryuji Suzuki, John R. Buck (Dept. of Elec. and Computer Eng. and Ctr. for Marine Sci. and Tech., Univ. of Massachusetts, North Dartmouth, MA 02747), and Peter L. Tyack (Woods Hole Oceanogr. Inst., Woods Hole, MA 02543)

Many theories of nonhuman animal communication posit a first-order Markov model in which the next signal depends only on the current one. Such a model precludes a hierarchical structure to the communication signal. Information theory and signal processing provide quantitative techniques to estimate the underlying complexity of an arbitrary signal or symbol sequence. These techniques are applied to humpback whale songs and demonstrate that any first-order Markov model fails to attain the underlying bound of complexity in these songs. Humpback songs are symbolized into alphabet sequences using spectrograms and self-organizing neural nets [Walker, unpublished]. The entropy of the song sequence is measured with a first-order parametric Markov model, and with a nonparametric sliding window method [Kontoyiannis et al., IEEE Trans. Info. Theory 44, 1319-1327 (1998)]. Preliminary analyses suggest that the entropy of the first-order Markov model is significantly higher than that of the nonparametric model, meaning that any first-order Markov source cannot reasonably model humpback songs. Furthermore, it is found that the symbolized song statistics are locally but not globally stationary, implying that these songs possess a hierarchical structure. [Work supported by NSF Ocean Sciences.]

\section{3:40}

2pAB6. Ultrasound-mediated transformation of prokaryotic cells. Jane A. Young (CDNSWC, Code 725, Bethesda, MD 20084 and COMB, Ste. 236, Columbus Ctr., 701 E. Pratt St., Baltimore, MD 21202), Joseph A. Clark (CDNSWC, Bethesda, MD 20084 and COMB, Baltimore, MD 21202), and William R. Jones (Univ. of Maryland, Baltimore, MD 21202)

Ultrasound-mediated transfection of mammalian cells has been reported in the literature [Kim et al., "Ultrasound-mediated transfection of mammalian cells," Human Gene Therapy 7, 1339-1346 (July 10, 1996)]. Optimum results were obtained using continuous wave insonification at a carrier frequency of $\mathrm{MHz}$, peak pressures of 319-411 kPa, exposure times of 20-60 s, and ambient temperatures $23-37^{\circ} \mathrm{C}$. Cavitation was shown to be a key mechanism for the insertion of plasmid DNA into cells. Prokaryotic cells appear to present a greater challenge to ultrasound approaches because of their smaller size and stronger membranes. This talk will report results of a series of ultrasound-mediated transformation experiments on prokaryotic cells, specifically bacteria. Conditions required to obtain a lethal dose for $50 \%$ of the cells (LD50) and transformation of cells will be presented. Effects of peak pressure levels, pulse duration, quiescent time between pulses, static pressure, and number of exposures will be discussed. This work could have broad applications, from disinfection of aquacultural and agricultural systems to genetic engineering.

\section{4:00-4:20 Break}

\section{4:20}

2pAB7. On interfacing physics with electrophysiology in echolocation in bats. Daniel R. Raichel (Dept. of Mech. and Aerosp. Eng., CUNY Grad. School, City College of CUNY, New York, NY 10031, raichel@juno.com) and Mathew J. Palakal (Indiana Univ.-Purdue Univ. at Indianapolis, Indianapolis, IN 46202)

In animal echolocation, reflected signals carry far more information than is generally realized. The real, nonlinear nature of sound propagation and the sensitivity of reflected sound to the effects of the target and its environs provide a matrix $M$ representative of both probe and reflected signals that lends itself to real-time spectral processing and formation of acoustical images. This process necessitates a sensitivity to signals at least two or more octaves beyond the fundamentals of the probe signals, combined with a high degree of resolution in the frequency, signal strength, and phase domains. The information feeds into the neurological system at the rate of $d M / d t$, enabling neurological processing of the high-frequency downward pulses ranging from 20 to $100 \mathrm{kHz}$ used by echolocation bats as well as pure FM pulses and CF-FM combination pulses used by certain species of bats. The multi-dimensional matrix also accommodates the duration and repetition rates of these pulses which are continuously altered according to the perceptual demand during echolocation. Electrophysiological data suggests that the specialized delay-sensitive neurons that are present in various regions of the auditory pathway allow the animal to obtain fine delay acuity and target discrimination ability in cluttered environments, with nanosecond resolution.

\section{$4: 40$}

2pAB8. Biosonar in a textured world. Rolf Müller and Roman Kuc (Intelligent Sensors Lab., Elec. Eng. Dept., Yale Univ., New Haven, CT 06520-8284, rolf@isl.eng.yale.edu)

Capabilities of bats to analyze spectral profiles have been demonstrated by several researchers. However, suggestions as to the informational content of the biologically accessible features in terms of the echogenerating process are not yet entirely convincing. Invoking a general analogy to visual color perception does not provide an operational definition of the hypothetical perceptual mechanism. The proposed mechanisms are mostly restricted to a scenario, where the spacing of two reflectors may be determined via the resulting comb-filter transfer function. The generality of this concept is curtailed by most natural surfaces constituting aggregates of multiple reflecting facets with a pronounced random component to their arrangement. Classification of natural textures is conceived to be a pivotal capability which may underly performance in several tasks probably performed by the animals, e.g., landmark identification and control of scanning movement. In order to understand how the demands of texture classification relate to the key features of biological sonar systems, the following steps are taken: Starting from a database of textures typical of bat's habitats (i.e., foliage), the implications of two paramount attributes of biological sonar systems are studied: sensor mobility and a joint timefrequency representation encoded in spike patterns. 
2pAB9. Modeling the role of nonhuman vocal membranes in phonation. Hanspeter Herzel (Inst. Theor. Biol., Humboldt Univ., Invalidenstr. 43, D-10115 Berlin, Germany, h.herzel@biologie.hu-berlin.de), Patrick Mergell (Univ. Erlangen-Nuremberg, Bohlenplatz 21, D-91054 Erlangen, Germany), and Tecumseh Fitch (Harvard/MIT Speech and Hearing Sci., Cambridge, MA 02138)

Although the mammalian larynx exhibits little structural variation compared to sound-producing organs in other taxa (birds or insects), there are some morphological features which could lead to significant differences in acoustic functioning, such as air sacs and vocal membranes. The vocal membrane (or "vocal lip") is a thin upward extension of the vocal fold that is present in many bat and primate species. The vocal membrane was modeled as an additional geometrical element in a two-mass model of the larynx. It was found that vocal membranes of an optimal angle and length can substantially lower the subglottal pressure at which phonation is supported, thus increasing vocal efficiency, and that this effect is most pronounced at high frequencies. The implications of this finding are discussed for animals such as bats and primates which are able to produce loud, high-pitched calls. Modeling efforts such as this provide guidance tion, can provide insight into the mechanisms of animal communication, and could potentially lead to better understanding of human clinical disorders such as sulcus vocalis.

2pAB10. Middle ear frequency characteristics of Norwegian cattle. Magne Kringlebotn (Dept. of Phys., Norwegian Univ. of Science and Technol., N-7034 Trondheim, Norway)

Preliminary results indicate that for a constant sound pressure to the tympanic membrane, the mean low-frequency asymptote value for the volume displacement in the inner ear windows of Norwegian cattle as compared to human ears is less by about $8 \mathrm{~dB}$, while above about $2 \mathrm{kHz}$ the volume displacement becomes increasingly larger than in the human ear. If mean amplitude responses are roughly approximated by straight lines above $1 \mathrm{kHz}$, the slope for cattle ears is about $-4 \mathrm{~dB} / \mathrm{oct}$ as compared to an earlier measured value of $-16 \mathrm{~dB} /$ oct for the human ear. Mean resonance frequencies are nearly equal, both about $0.8 \mathrm{kHz}$. The phase of the windows volume displacement relative to the sound pressure at the tympanic membrane is close to zero below $0.2 \mathrm{kHz}$, and falls to about $-4 \pi$ at $20 \mathrm{kHz}$. Earlier phase measurements in human ears indicating a phase change of less than $2 \pi$ are believed to be in error.

TUESDAY AFTERNOON, 16 MARCH 1999

ROOM H1058, 2:00 TO 6:00 P.M.

Session 2pAO

\title{
Acoustical Oceanography: Innovations in Fish and Plankton Acoustics II
}

\author{
Chris Feuillade, Cochair \\ Naval Research Laboratory, Stennis Space Center, Mississippi 39529-5004, USA \\ David N. MacLennan, Cochair \\ Marine Laboratory, P.O. Box 101, Victoria Road, Aberdeen AB11 8DB, UK
}

Contributed Papers

\section{2:00}

2pAO1. High-resolution target strength measurements in deep water. Egil Ona and Ingvald Svellingen (Inst. of Marine Res., P.O. Box 1870, 5024 Bergen, Norway, egil.ona@imr.no)

High-resolution target strength data can only be claimed valid in situations where it can be safely shown to be much less than one target per pulse volume. Many fish species occur in densities and at depths where this demand hardly can be met with standard, hull-mounted, survey transducers. This paper describes a new and simple method for obtaining such data with some examples of target strength and target tracking data obtained in deep water at three different cruises. The system used is the Simrad EK-500 split beam echo sounder, connected to an oil-filled ES38D, pressure-resistant transducer, on cable lengths from 400-800 m. The transducer was lowered as a probe the desired depth, often inside or close to the fish layer, with the vessel stationary, or slowly drifting. For maximizing the number of detections per fish, the echo sounder was operated at maximum pulse repetition frequency. Recordings shown are from three different species, small myctophid fishes at 200-400 m, hake at 200-400 m, and herring at 50-400 m. High-quality target strength distributions were frequently obtained in less than $1 \mathrm{~h}$ at a typical target strength station.

\section{2:20}

2pAO2. A summary of target strength observations on fishes from the shelf off West Africa. Ingvald Svellingen and Egil Ona (Inst. of Marine Res., P.O. Box 1870, 5024 Bergen, Norway, egil.ona@imr.no)

In many areas, and in tropical waters in particular, it may be difficult to fulfill the resolution criteria for high-quality target strength measurements both with respect to density and species mixing. Since 1985 target strength data have been collected periodically during cruises with R/V DR. FRIDTJOF NANSEN in West African waters. As a first attempt to establish a reasonably correct target strength for important species in the area, swimbladder morphology was studied and compared with species from the North Atlantic. After 1986, in situ target strength data was collected off Morocco and in Namibian waters using split-beam echo sounders. Data from the following species have been analyzed and discussed: Pilchard (Sardina Pilchardus), horse mackerel (Trachurus Capensis), hake (Merluccius Capensis), and myctophids. Only data which were considered to be of high quality have been analyzed. The calculated average target strength for pilchard are several decibels higher than the currently applied target strength of North Sea herring, but not very different from other reported target strength of sardine in other areas. Also, the target strength of horse mackerel is high compared to the presently applied target strength. For hake, the measured target strength are comparable to reported data for gadoids. 
chambers), as two independent chambers, and as two acoustically inter-

2pAO3. Resonance frequencies of absorption lines due to pelagic fish. Orest Diachok (Naval Res. Lab., Washington, DC 20375)

Resonance frequencies, $f_{0}$, of absorption lines due to pelagic fish with swim bladders change at twilight in accord with systematic changes in their depths and separations. Measurements of $f_{0}$ at night, and calculations of $f_{0}$, based on measurements of the effective radius, $r_{0}$, and eccentricity, $\varepsilon$, of sardine swim bladders, and the average depth of dispersed sardines at night, are in good agreement. This result implies that swim bladder compression by internal organs did not have a large effect on $r_{0}$ and $\varepsilon$ during this experiment. Close agreement between laboratory measurements and calculated values of $f_{0}$ of individual physotomes suggests that this inference is generally valid. Possible exceptions to this "rule" will be considered in the context of Ona's (1990) laboratory observations of the effects of internal organs on swim bladder compression, and measurements of the state of internal organs of fish in the ocean. The resonance frequencies of absorption lines attributed to sardines in schools, $f_{0}^{\prime} \approx 0.6 f_{0}$. This result is consistent with a modified form of d'Agostino and Brennan's (1988) equation for the fundamental mode of a bubble cloud. Their equation accounts for realistic numbers of fish per school $\left(10^{4}-10^{5}\right)$. Modifications, which account for realistic separations between fish in schools and school eccentricity, will be presented. [Work supported by ONR.]

\section{3:00}

2pA04. Modeling the target strength of Calanus finmarchicus. David T. I. Francis (School of Electron. and Elec. Eng., Univ. of Birmingham, Edgbaston, Birmingham B15 2TT, UK, francist@ee-admn.bham.ac.uk), Kenneth G. Foote, Tor Knutsen (Inst. of Marine Res., N-5024 Bergen, Norway), and Lucio Calise (Centro Marino Internazionale, I-09072 Torregrande (OR), Italy)

The boundary element method is applied to the copepod Calanus finmarchicus, treated as a composite body, with fluidlike oil sac embedded in a fluidlike, mostly transparent prosome. The generally complex shapes of the two bodies are modeled on the basis of the actual dorsal- and lateralaspect cross sections, as observed by videomicroscopy with the living, unanaesthetized animal encased in a droplet of sea water. Physical properties of the two bodies, namely mass density and longitudinal-wave sound speed, are derived through a combination of measurement and inference. Computations of backscattering cross section as a function of orientation and frequency are presented over the range $25 \mathrm{kHz}$ to $3.2 \mathrm{MHz}$ for a number of specimens. A sensitivity analysis is performed to quantify some uncertainty in the assumed values of the physical properties. [Support by the following is acknowledged: EU through RTD Contract No. MAS3-CT95-0031, Norwegian Research Council through Grant No. 113809/122, and Bergen Large-Scale Facility (LSF) for Marine Pelagic Food Chain Research.]

\section{3:20}

2pA05. Comparing predictions from backscatter models to in situ measurements of a dual-chambered, swimbladdered fish. John K. Horne, J. Michael Jech (CILER, Univ. of Michigan, 2205 Commonwealth Blvd., Ann Arbor, MI 48105, horne@glerl.noaa.gov), and Paul D. Walline (Israel Oceanograph. and Limnological Res., Tiberias, Israel 14102)

Most acoustic backscatter models predict echo amplitudes of single or aggregations of fish with single-chambered swimbladders. Lavnun (Acanthobrama terraesanctae), a planktivorous fish in Lake Kinneret, Israel, possesses a dual-chambered swimbladder. Target strength, length, frequency, or aspect relationships do not exist for this commercially important species. What is the most appropriate way to model backscatter amplitudes from single and aggregations of fish with multi-chambered swimbladders? Carrier frequency, organism length, organism aspect, and swimbladder shape all influence amplitudes of returned echoes. Predicted backscatter amplitudes and intra-species variance predictions from an anatomically-based Kirchhoff-ray mode model were compared to in situ measures of lavnun backscatter at 120 and $420 \mathrm{kHz}$. The lavnun swimbladder was modeled as a single chamber (with equivalent volume of both acting chambers. Wider application and comparison of theoretical models to field measurements increases understanding of factors influencing backscatter amplitude and variability for all fish species, and should improve methods for identifying organisms. [Work supported by ONR and USIsrael BSF.]

\section{$3: 40$}

2pAO6. Comparison of Ayu target strength estimates determined from tank measurement and calculations. Kouichi Sawada (Natl. Res. Inst. of Fisheries Eng., Ebidai, Hasaki, Kashima, Ibaraki, 314-0421 Japan, ksawada@nrife.affrc.go.jp), Masahiko Yoshida (Mie Univ., Mie, 514-8507 Japan), Yoichi Miyanohana (Natl. Res. Inst. of Fisheries Eng., Ibaraki, 314-0421 Japan), and Kunio Shirakihara (Mie Univ., Mie, 514-8507 Japan)

Ayu, Plecoglossus altivelis, is one of the most popular freshwater fish which can be seen in many rivers in Japan. Especially in the North Basin of Lake Biwa, Ayu is the highest predator. It is important to know the spatial and temporal variations of Ayu biomass to understand the predator's effect to the food chain in the lake. Acoustic survey has been conducted since 1995. There is little information about the target strength (TS) of Ayu. Since Ayu has an open swimbladder, it is necessary to measure TS before the swimbladder was deflated by the suspension method in a tank. Target strength of live Ayu was measured in a tank and a three-dimensional image of swimbladder was obtained by an x-ray computer tomography (CT) system. In addition, a side and a dorsal image of the swimbladder were obtained by a soft x-ray system. All measurements were done in live condition. Kirchhoff approximation was used for the calculation on the basis of the three-dimensional image of swimbladder. A deformed cylinder model and a prolate spheroid model were also used for the calculation on the basis of side and dorsal image of the swimbladder. Calculation and measurement are compared and discussed in this paper.

\section{4:00-4:20 Break}

$4: 20$

2pA07. Analyzing ship-borne acoustic Doppler current profiler measurements to estimate the vertical and spatial distribution of southern Black Sea zooplankton. Funda Erkan and Ali C. Gucu (Inst. of Marine Sci., Middle East Tech. Univ., P.O. Box 28, Erdemli, 33731, Icel, Turkey, funda@ims.metu.edu.tr)

The acoustic Doppler current profiler was used in Summer-Autumn 1996 and 1997 cruises of R/V BILIM of Middle East Technical University to study the distribution of zooplankton in the southern Black sea. The backscattered signal intensity is compared with macro and mesozooplankton samples collected with Nansen closing net and 30-L Niskin water samplers. Analyzing the taxonomic groups and the length distribution of the individuals in the samples, size classification has been done. Total cross-sectional areas of the zooplankton in the length classes were compared with the resultant MVBS values. Highly significant correlations were observed. The relations between acoustic units and the size classes of zooplankton were presented by an empirical model which described a high percent of the total variance. The data sets from basin-wide surveys were used to obtain spatial distribution of zooplankton in the southern Black Sea. The spatial distribution of zooplankton was to a great extent determined by hydrography of the Black Sea. Daily migration patterns of zooplankton were studied, and the descending and ascending rates of different zooplankton groups were also calculated. 
2pAO8. Analysis of the neuro-fuzzy classifiers for fish species identification from acoustic echoes. Andrzej Stepnowski, Dung Tran Van, and Joanna Maciolowska (Tech. Univ. of Gdansk, Acoust. Dept., 80-952 Gdansk, Poland, astep@pg.gda.pl)

Some of the variety of classification methods have been recently implemented also for fish species identification from their acoustic echoes. The classification systems employing artificial neural networks and fuzzy logic separately are not satisfactory, so the combined neuro-fuzzy approach were carried out. A couple of neuro-fuzzy classifiers were built and tested using the NEFCLASS program, which discovered the rules and found the shape of membership functions for determining correct class categories for a given input data set. The systems were tested on fish echoes acquired by DT4000 digital echo sounder. The results are promising with reference to the classifiers performance and their generalization abilities.

\section{5:00}

2pA09. Improving echograms' resolution by wideband pulse compression and dynamic focusing. Manell E. Zakharia (CPE Lyon, LASSSO (LISA, EP92 CNRS), 43 Bd. du 11 Novembre 1918, BP 2077, Bat. 308, F 69616, Villeurbanne Cedex, France)

Improving the resolution of fisheries' sounder echograms (i.e., reducing the confusion volume) can be achieved by improving either the axial or the angular resolution. On one hand, the range (i.e., time) resolution can be improved without any degradation of the signal-to-noise ratio by using frequency-modulated signals associated with pulse compression. Axial resolution can be considerably enhanced by using wideband signals. On the other hand, due to the angular spreading of the sonar beam, the lateral resolution is proportional to the range. Improving the angular resolution can only be achieved by using a larger array (only possible on the receiving side). Nevertheless, such a solution has a severe limitation as it reduces the volumes sampled at short range, leading to an angular undersampling of the biomass. Dynamic focusing can be used to overcome this problem. Its principle is simple: the shorter the range, the smaller the array (i.e., the larger the angular aperture). The use of such a technique needs a compromise between the acceptable beam divergence and the system complexity. Both techniques will be applied to real data and echograms will be displayed and compared to the ones obtained with a standard system.
2pAO10. Bergen acoustic buoy (BAB) - A tool for remote monitoring of marine resources. Olav Rune Godo and Atle Totland (Inst. of Marine Res., P.O. Box 1870, 5024 Bergen, Norway)

Marine resource abundance monitoring is often accomplished by means of research vessel acoustic surveys. Vessel time is limited and expensive, and optimal and cost-efficient utilization is required. Such vessels have clear limitations in observing the true acoustic distribution, density, and acoustic properties of marine organisms due to noise and light emitted during surveying and sampling. A potential solution to some of the emerging problems is a remote operated acoustic device, which can be used to monitor distribution, density, movements, and properties of marine organisms under natural and survey vessel affected conditions. In this paper a new concept for a remote operated acoustic data logger is presented which can give an online presentation of the acoustic information. The system is built on the new Simrad General Purpose Transceiver (GPT) operated through a standard windows NT interface. The technical specification and operational features will be presented and exemplified with experience from the first sea trials. The system is designed to meet demands from other applications, e.g., a drop sonde and towed underwater function, and future development of the concept is discussed.

\section{$5: 40$}

2pAO11. Design of a transducer-driving system scanning around an aquatic target in an indoor tank. Ken Ishii, Tsuyoshi Okumura, Kouichi Sawada, and Yoichi Miyanohana (Natl. Res. Inst. of Fisheries Eng., Ebidai Hasaki, Kashima, Ibaraki, 314-0421 Japan)

A squid has been specified as a kind of fish that has to been managed scientifically. Estimation of its resources via acoustic surveys is required. But the basic data of the target strength (TS) of a squid are limited. So far, in the measurements of the TS pattern of fish, echo levels are observed while changing the aspect of a fish. On the other hand, since a squid is more fragile than a fish, and unstable in shape, the rotating unit of fish is not available for a squid. So the positioning system that makes a transducer scan precisely around an aquatic target suspended in the water has been designed. This system is loaded in the electric flatcar, the probe of which can move three dimensionally, on the top of a tank which is $10 \mathrm{~m}$ in width, $15 \mathrm{~m}$ in length, and $10 \mathrm{~m}$ in depth. It is hoped that precise TS pattern measurements are possible so that the target is suspended without damage owing to this transducer-driving system. The detail of control software and an example of measurements and subjects are described in this paper. 
Session 2pEA

Engineering Acoustics: Microphones, Measurements, Etc.

\author{
George S. K. Wong, Cochair \\ Institute for National Measurement Standards, National Research Council, Montreal Road, Ottawa, Ontario KIA OR6, \\ Canada \\ Gunnar Rasmussen, Cochair \\ G.R.A.S. Sound \& Vibration aps, Skelstedet 10B, 2950 Vedbaek, Denmark
}

Chair's Introduction-1:55

Contributed Papers

2:00

2pEA1. A microphone without a membrane. Joerg Sennheiser (Sennheiser Electron. GmbH \& Co. Kg, Am Labor 1, 30900 Wedemark, Germany)

In all microphones used today, the component which affects the quality most is the membrane. The frequency response and the signal-to-noise ratio of a microphone depend on the best possible coupling of this mechanical component to the acoustic sound field and on its oscillatory behavior. In the mass production of membranes within narrow tolerances, properties such as geometry, mass, stiffness, native modes, etc. have a great effect on the quality and are often problematic. There is therefore great interest in finding a working principle for microphones which does not require a membrane. A possible way out is to utilize the well-known thermal anemometer principle: An electrically heated wire is cooled down in an air flow. The resistance change in the wire is a measure of the air speed and thus of the velocity. If the heat capacity of the heated sensor is reduced by micromechanical techniques, this principle can also be used to measure the acoustical volume velocity at higher frequencies. The expected limits and first readings are shown via the models realized.

\section{2:20}

2pEA2. A simple optical microphone. Wolfgang Niehoff (Sennheiser Electron. GmbH \& Co. Kg, Am Labor 1, 30900 Wedemark, Germany)

In the past, a number of different working principles have been suggested for optical microphones, but many of these require a high degree of technical sophistication. Thus some suggestions are based on the interferometric principle, whilst others would utilize frequency modulation of laser light via a variable mirror (membrane) situated in the laser resonator. Light intensity modulation is a much more simple approach: A light beam emitted from an optical fiber is aimed at a reflecting membrane. The beam reflected from the membrane is captured by a second optical fiber and fed into a photodiode. Depending on the movement of the membrane, more or less light is launched into a second optical fiber. The photoelectric current of the photodiode thus expresses the movement of the membrane, and with it the sound field, as an electrical signal. As optical fibers transmit light with very few losses, the actual optoacoustic transducer can be positioned at a long distance from the light source and the photodiode. This allows problem-free use of the optoacoustic transducer in the presence of very strong electromagnetic fields (e.g., MRI). It is possible to improve upon this principle via simple modifications. The electroacoustic characteristics achieved are discussed and a model is presented.
$2: 40$

2pEA3. Microphone calibration techniques. Gunnar Rasmussen (G.R.A.S. Sound \& Vib. aps, Skelstedet 10B, Vedbaek, Denmark)

Primary calibration of microphones is well described and standardized by IEC 1094-2 using the reciprocity technique. When the standard microphone is calibrated, a reference source is needed for transfer of the calibration value to the acoustical measuring devices or systems employing the working measurement microphone. A reliable transfer device is the pistonphone. Another method may be the use of a comparison against a working standard microphone. Sound reference sources called "calibrators" are standarized by IEC in 60942:1997. Sound calibrators may often be inferior in accuracy and stability to the systems they are being applied to. They should therefore be labelled check devices. Other techniques have been employed for many years. The use of a reference voltage coupled to the system input is widely used. This may be extended to incorporate the preamplifier and incorporate features like modulating the polarization voltage whether internal or external or incorporating the cartridge capacity into a capacitive voltage divider. These techniques do not allow a calibration but only supply a means of injecting a signal in the electrical system to check the ability to transfer a signal. Calibration is not obtained.

3:00

2pEA4. Optical measuring and calibration techniques for microphones. Gottfried K. Behler and Michael Vorländer (Inst. of Tech. Acoust., Univ. of Aachen, Templergraben 55, 52056 Aachen, Germany)

Microphone measurement techniques are normally based on acoustical excitation and electrical derivation of either the voltage or the current output of the transducer. With condenser microphones, electrical excitation can be used as well. Calibration of microphones based on acoustical excitation therefore is quite time consuming, and sensitive for several errors originating in inaccurate sound-field properties and environment conditions. Measurements of the microphone membrane velocity with optical means like the laser Doppler vibrometer technique offer an accurate, touchless method to derive the local movement at almost each point of the membrane. Excitation of the membrane can be accomplished by direct feeding of the transducer (due to its reversible construction), which provides highest accuracy for the derivation of either current or voltage at the terminals of the transducer. From the knowledge of the vibration characteristics and the excitation properties, the "differential transducer sensitivity" defined at each surface point can be derived. With numerical methods, like the discrete solution of the Helmholtz equation or BEM, the radiated sound can be calculated, and finally the overall microphone radiation pattern and sensitivity can be calculated. The described techniques were applied to different types of microphones. The measurement and calculation results will be shown. 
2pEA5. New design concepts for the construction of an omnidirectional sound source. Christos A. Goussios, George V. Papanikolaou (Dept. of Elec. and Computer Eng., Aristotle Univ. of Thessaloniki, Univ. Campus 540 06, Thessaloniki, Greece), and George M. Kalliris (Aristotle Univ. of Thessaloniki, Thessaloniki, Greece)

This paper presents a study for the optimum design and construction of an omnidirectional point source. The point source model of theoretical acoustics is used as an elementary unit for electroacoustic measurements in the area of room and free-field acoustics. The spherical radiation requirement for the entire acoustic spectrum and the finite dimensions of the point source model contradict the construction in practice. This is because of high-frequency beaming and the existing dimensions of radiators. A new design approach of using horn loaded cone loudspeakers and properly placed high-frequency diffusers was used to minimize these problems. Twelve radiators loaded on pentagon-shaped horns were placed in a dodecahedron topology to build a practical point source with a useful frequency response of $80-16000 \mathrm{~Hz}$ and an omnidirectional radiation pattern up to $12000 \mathrm{~Hz}$. The results display improvement in the radiation pattern compared to those of the already existing point sources, due to the use of horns and diffusers. Increase in the sound pressure level is also displayed because of the obvious increase of the directivity index of each individual radiating element.

\section{3:40}

2pEA6. Simulation of ultrasonic fields in composite media with planar boundaries. Liudas Mažeika and Rymantas J. Kažys (Ultrasound Res. Ctr., Kaunas Univ. of Technol., Studentu̧ str. 50, LT-3031, Kaunas, Lithuania, liudas.mazeika@tef.ktu.lt)

In this paper the method and fast algorithm for simulation of ultrasonic fields exited by the direct or angular transducers in media with a few thick layers are presented. The method exploits regularities of the disk-shaped transducer field in a homogeneous medium, adapting it to a multilayered medium, the boundaries of which are not necessarily parallel. It was assumed that at any point the pulse response of the transducer must be of the similar shape, consisting of the fragments corresponding to the plane and the edge waves. The additional parameters of the pulse response are evaluated solving a system of nonlinear equations. These parameters are used to find some equivalent point in pulse response for a homogeneous medium with adequate values of parameters. The method proposed allows the evaluation of ultrasonic field structure in the solids when the immersion technique is used or inside tanks when ultrasonic waves pass through a wall. The simulation and experimental results presented show a good correspondence with the field structure calculated using other methods.

\section{4:00}

2pEA7. Wideband ultrasonic piezoelectric microphones. Vygantas Augutis and Darius Gailius (Varpo 10-12, Kaunas 3028, Lithuania, dagail@tef.ktu.lt)

Microphones with an air coupling multiple matching layer and fiber damper were developed. The method for calculation of the characteristics of the transducers is presented. The signal-to-noise ratio (SNR) of these microphones is about $10 \mathrm{~dB}$ lower than the SNR ratio of capacitive microphones, but they are more suitable for use in dusty and wet conditions. The frequency range of these microphones is $10-100 \mathrm{kHz}$. The system for the measurement of the characteristic of microphones was developed. Short acoustic pulses, generated by the electical spark, were used as a wideband probing acoustic signal. Serious attention was paid to the estimation of instability of the spark-generated acoustic pulses. Several modifications of developed microphones, their characteristics, and fields of application are presented.

\section{$4: 40$}

2pEA8. A simple method to estimate the absorption coefficient of porous absorbers. Ulrich Ackermann (Dept. of Mech. Eng., Märkische Fachhochschule Iserlohn, Postfach 2061, D-58590 Iserlohn, Germany)

To estimate the attenuation $D$ of silencers under practical conditions, the formula of Piening is used: $D=1.5 \alpha C L / A$ ( $C$ is the circumference, $L$ the length, and $A$ the cross-sectional area of the absorbing duct). For most of the porous absorbers the absorption coefficient $\alpha$ can be estimated by the very simple relation $\alpha=0.9 \log (f d)-X(0 \leqslant \alpha \leqslant 1)$, where $f$ is the frequency in $\mathrm{Hz}$ and $d$ the thickness of the absorber in $\mathrm{cm}$. For different porous absorbers the constant $X$ can be derived from measurements in small test rigs, where only a few materials are needed. For mineral wool with a density between 20 and $40 \mathrm{~kg} / \mathrm{m}^{3}, X=2,4$, and for snow with a density of $270 \mathrm{~kg} / \mathrm{m}^{3}, X=2,65$, e.g., the Piening formula together with the relation for $\alpha$ gives a simple method to calculate the space for splitteror tube-silencers in ducts, e.g., without knowing the acoustical performance of the porous absorber exactly.

\section{5:00}

2pEA9. A simple device for flow resistance measurements. Dag Holmberg and Erling Nilsson (Dept. of Eng. Acoust., LTH, Lund Univ., P.O. Box 118, SE221 00 Lund, Sweden, Dag.Holmberg@kstr.lth.se)

A simple device for static measurements of flow resistance on thin porous materials is developed and evaluated. The device consists of a duct with a piston which is driven at a steady low speed. This generates a flow of air with constant volume velocity. The duct ends with a cavity with an interface to the outside where the sample is mounted. The differential pressure between the cavity and the outside is measured. In order to prevent nonlinear effects, the speed of the piston is kept so low that the constant velocity of the air is of the same order of magnitude at the sample as the rms velocity of sound waves at approximately $100 \mathrm{~dB}$ SPL. The device is so constructed that it is possible to measure samples of standard dimensions, e.g., cloth mounted at different perforated frames, if the speed of the moving piston is accordingly adjusted to match the open area. The performance of the device is tested and discussed.

\section{$5: 20$}

2pEA10. Experimental investigation of bending waves in ultrasonic reactors. Torsten Niederdraenk (Siemens Audiologische Technik GmbH, D-91050 Erlangen, Germany) and Rainer Thaden (Institut fuer Technische Akustik, RWTH Aachen, D-52056 Aachen, Germany)

A number of practical applications like ultrasonic cleaning, sonochemistry, and others use the effects of high, intense ultrasound. In general, the high-pressure sound fields can be obtained by different arrangements of piezoelectric compound transducers, which have to be optimized according to each application. A commonly used setup is a planar array transducer, consisting of one or more transducer elements. A tool to design and develop the transducer arrangements is given by simulations of the electromechanical equivalent circuit, although the results are often based on several assumptions. This paper reports on vibroacoustic investigations of compound transducer arrangements. Laser-optical measurements give evidence for arising bending waves on the radiating surface of the transducers that may affect the generated sound field and which have some effect on the total power distribution. The influence of bending waves has been included in the equivalent circuit; some simulations are presented. 
2pEA11. Underwater acoustic tank evaluation of acoustic properties of samples using spectrally dense signals. Carlos Ranz-Guerra and Pedro Cobo-Parra (Inst. de Acstica, C/Serrano 144, 28006, Madrid, Spain, iacrg32@fresno.csic.es)

This paper deals with exploring the availability and reliability of subtraction techniques, based on spectrally dense signals such as maximum length sequences, in evaluating the echo reduction losses and transmitting properties of samples of interest in underwater acoustics. These techniques, first employed in air acoustics, were adapted to the underwater environment. Experimental results show how a measurement can be accomplished and the limitations produced by the actual available combination of soft, hardware and tank and samples dimensions. [The authors wish to acknowledge the help given by the CICYT, Project AMB97-1175-C0301 , for allowing them to use the software, hardware, and related facilities in carrying out the work described by this paper.]
2pEA12. Mechanical impedance of wheeled tractor steering mechanism. Stanislovas Merkevičius and Ričardas Vagusevičius (Lithuanian Univ. of Agriculture, Agriculture Eng. Faculty, Occupational Prevention and Eng. Management Dept., Kaunas-Acad. 4324, Lithuania)

There are fairly many wheeled tractors of $6 \mathrm{kN}$ class made in CIS (Community of Independent States). The characteristics of noise and vibration are inadequate for valid standards. Vibrations of especially high levels having the effect on hands are generated through the steering wheel to the person's (operator's) hands. If fitting in vibroisolating or vibrodepherisating elements to the driving mechanism details junctions (joints), it is partly possible to lessen the vibration level having the effect on the person. It would be more effective and more rational to discuss the possibilities of absorbing spreading vibration and vibroisolation when there is enough information about the vibration of the steering wheel mechanism. Applying the methods of mechanical impedance, the mathematical model of steering mechanism, consisting of the steering wheel mechanism and the steering wheel has been made, giving an opportunity to analyze the interaction of the steering wheel, the person (operator), and the spreading vibration.

TUESDAY AFTERNOON, 16 MARCH 1999

ROOM MA004, 1:55 TO 6:20 P.M.

\title{
Session 2pMU
}

\section{Musical Acoustics: Musical Acoustics and the Musician}

\author{
Thomas D. Rossing, Cochair \\ Physics Department, Northern Illinois University, DeKalb, Illinois 60115, USA
}

Anders Askenfelt, Cochair

Department of Speech, Music, and Hearing, Royal Institute of Technology, P.O. Box 70014, 10044 Stockholm, Sweden

\author{
Chair's Introduction-1:55 \\ Invited Papers
}

2:00

2pMU1. The perception of music by the human brain: An introductory course. Juan G. Roederer (Geophysical Inst., Univ. of Alaska-Fairbanks, Fairbanks, AK 99775-7320)

The study of the perception of music is a fascinating interdisciplinary endeavor in which musicians, neuroscientists, psychologists, physicists, and physiologists work together. Recent advances in the understanding of how musical sounds are detected in the ear, converted into neural signals, processed, and interpreted in the brain make the subject very attractive for an elective introductory university course. It is possible to present many subject matters in a generally understandable way, without the requirement of previous knowledge beyond that of secondary level [J. G. Roederer, Physics and Psychophysics of Music, 3rd ed. (Springer-Verlag, New York, 1995); German edition: Physikalische und Psychoakustische Grundlagen der Musik (Springer-Verlag, Heidelberg)]. In such a course, many puzzling questions can be addressed (and answered!): Why do people perceive only one pitch when they listen to a musical tone made up on many harmonics? Why are there musical scales? Why is pitch resolved so well, despite the "bad" frequency analysis in the inner ear? What happens in the brain when sounds are imagined? What is the relationship between music and language? Why do listeners react emotionally to musical sounds? The pedagogical challenges of organizing such an interdisciplinary course will be discussed.

2pMU2. Musical acoustics, the music student, and the music teacher. Knut Guettler (Norwegian State Acad. of Music, P. B. 5190 Majorstua, 0302 Oslo, Norway, knut.guettler@samson.nmh.no)

Dialogs between musicians and acousticians are not always successful. A lack of common language combined with mutual suspicion of the other person trespassing on ones own professional authority, often restricts what ought to have been a fruitful exchange of knowledge. For the music student, there is, however, a third matter that seriously impedes the possibility of gaining acoustical insight. As for all arts, yearning and intuition are the driving forces in the development of musical and instrumental skills. 
Focus on purely technical matters, or even worse, the feeling of being overruled by such, is hardly combinable with a desirable musical progress of the young student. Based on the author's experience as a principal professional player and teacher of music for more than 30 years, this paper gives some suggestions on which and how topics in musical acoustics may be introduced.

\section{2:40}

2pMU3. Acoustical training for stringed instrument makers. Bernard E. Richardson (Dept. of Phys. and Astron., Univ. of Wales, Cardiff, P.O. Box 913, Cardiff CF2 3YB, UK, RichardsonBE@Cardiff.ac.uk)

The vast majority of popular stringed instruments found in western culture have been the product of generations of empirical experimentation on the part of craftsmen trying to provide a suitable vehicle for musical expression. In previous centuries, skills and knowledge were passed down from master to pupil during long apprenticeships, but whenever the continuity has been broken, there has been a feeling that various "secrets" have been lost. In more recent years, increased leisure time and better education has encouraged many individuals to take up instrument making, either as amateurs or as professionals. Most of these makers work in isolation, so there is now an even greater impetus to "rediscover" and communicate past arts. Some of these makers have turned to science to provide rational explanations of the many questions which are inevitably raised during the pursuit of making "fine" instruments. Based on the author's experience of his interactions with pupils of violin and guitar making, this paper seeks to explore how scientific investigations can best be communicated to makers and examines to what extent "scientists" and "makers" can learn from each other.

\section{3:00}

2pMU4. Intensive training on acoustics of pipe organs for organ builders and organists. Judit Angster and Andras Miklos (Fraunhofer-Institut Bauphysik, Nobelstr. 12, D-70569 Stuttgart, Germany, angster@ibp.fhg.de)

A special course on acoustics is organized regularly in the Fraunhofer-Institute fr Bauphysik (IBP) for training pipe organ builders, official organ experts, and organists. The title of the 4-day intensive course is "Pipe Organ and Church Acoustics" and it is scheduled at the end of January every year, when most of the organ builders are less occupied than in other periods. The number of participants is limited to 20, but the course is repeated in February, if necessary. The working language of the course is German, but English and French are also possible. The curriculum of the course is based on the ongoing research on pipe organs in the IBP and it is divided to three parts: fundamentals of acoustics; recent results of research on organ and church acoustics; and laboratory demonstrations and practical applications. During the demonstrations there is a limited possibility to perform measurements suggested by the participants. The content of the lectures and demonstrations is upgraded every year with the newest results of the research. In this paper the experiences of the eight courses held up to now will be discussed. A short video clip showing some of the laboratory demonstrations will also be presented.

2pMU5. Acoustic loading exercises in singing training. Ingo R. Titze (Dept. of Speech Pathol. and Audiol. and Natl. Ctr. for Voice and Speech, Univ. of Iowa, Iowa City, IA 52242, ingo-titze@uiowa.edu)

Singing teachers have devised vocal exercises that present variable acoustic loads to the sound source. These exercises consist of lip and tongue trills, nasal consonants, and vowels with small mouth openings (like /u/). Some pedagogues and clinicians have advocated phonating into long and narrow tubes. Since none of these exercises produce either a strong or aesthetically pleasing acoustic output in and of themselves, their merit must lie in facilitating the respiratory and phonatory systems. Recent Hopf bifurcation analysis has shown that inertive acoustic loads, resulting from deliberate constricting and lengthening of some portion of the vocal tract, can lower the oscillation threshold pressure, thereby facilitating the ease of vocal onset. Resistive acoustic loads, either upstream or downstream of the vocal folds, have a less beneficial effect on oscillation threshold pressure. [Work supported by Grant No. P60 DC00976 from the National Institute on Deafness and Other Communication Disorders.]

\section{$3: 40$}

2pMU6. Illustrating music performance principles by synthesis. Anders Friberg and Johan Sundberg (Royal Inst. of Technol., Dept. of Speech, Music and Hearing, Drottning Kristinas vg 31, SE-100 44 Stockholm, Sweden, andersf@speech.kth.se)

Music is generally not performed exactly as written. Instead, many small systematic variations of tone duration, pitch, articulation, etc. are found in a performance. These variations may serve the purpose of communicating different emotions or to outline the musical structure. An overview of commonly used principles is presented concerning the performance of different rhythmic patterns, the marking of important notes or chords, musical punctuation, and the hierarchical phrase structure. These principles are encoded in the form of rules implemented in a computer program, where they can be applied on any musical example. The possibility to isolate and change the quantity of the effects generated by each rule makes the system a unique tool for illustrating music performance to music students.

\section{4:00-4:20 Break}

\section{4:20}

2pMU7. Music acoustics and contemporary musical composition. Gerald Bennett (Swiss Ctr. for Comput. Music, Florhofgasse 6, 8001 Zurich, Switzerland, gbennett@cerfnet.com)

Although it may seem that in the past musical composition got by well without specific knowledge of music acoustics, in fact the acoustical knowledge of each epoch has always determined the representational framework within which the composer formulates his ideas. For the last 50 years, however, composition in the field of electroacoustic music has required far more explicit and detailed 
acoustical knowledge than was the case for instrumental music. The relationship between music acoustics and composition today is one of reciprocal influence: frequently research in music acoustics is put to good (if sometimes surprising) use by composers. Sometimes, however, composers' needs have led to important research in music acoustics. The present paper illustrates this reciprocity with examples from the areas of sound synthesis, physical modeling, and the simulation of reverberant spaces, and speaks of the importance of research for contemporary composition. In addition, the author presents a wish list for future work in music acoustics.

4:40

2pMU8. Teaching musical acoustics. R. Dean Ayers (Dept. of Phys. and Astron., California State Univ., Long Beach, CA 90840) and Thomas D. Rossing (Northern Illinois Univ., DeKalb, IL 60115)

Although designed particularly for music students, musical acoustics courses at American universities generally draw a "mixed bag" of students with a rather wide variety of backgrounds. This presents some real challenges but also some real rewards. Some of the problems in teaching musical acoustics to heterogenous classes and some of the ways these problems can be dealt with are discussed. Readable textbooks, demonstration experiments, and videotapes help to challenge and motivate the students, but the best way of all to learn the subject is by doing hands-on experiments.

\section{Contributed Papers}

5:00

2pMU9. Measurement of performance response differences when striking different areas of a marimba bar. Barry J. Larkin (Dept. of Music, Iowa State Univ., Ames, IA 50011, blarkin@iastate.edu) and Ronald A. Roberts (Iowa State Univ., Ames, IA 50011)

Over the past 40 years, general percussion education manuals have stated that the best place to activate a marimba or xylophone bar is to strike it in the exact center. Over the years, this has become known as the "best spot," and percussion students have been blindly advised to strive always to perform in this manner. Recent re-publications of mallet methods from the early part of the 20th century show that it was a common practice to perform on the end of the accidental bars for speed and superior sound. Also, leading classical marimba performer, Leigh Howard Stevens, has stated his preference for this type of execution. This paper will present spectral analysis results from measurements made at nine different locations on a Great F sharp marimba bar. The results will show the diverse types of responses generated by location changes of as little as an inch.

\section{$5: 20$}

2pMU10. Control index of the upright and grand piano actions. Xavier Boutillon (Laboratoire d'Acoustique Musicale, CNRS, Case 161, 4 place Jussieu, 75252 Paris Cedex 05, France) and Robert Grijalva (Univ. of Michigan, Ann Arbor, MI 48109-2085)

The motion of the piano hammer between its resting position and the contact with the strings follows two phases. At first, the motion is imposed by the pianist's finger on the key. When it escapes from the main action, within $2 \mathrm{~mm}$ from the string, the hammer possesses the velocity $v_{e}$. During the second and brief phase before impact, the hammer is only subject to gravity, friction of its pivoting axis, and to the action of an additional spring in the case of the upright piano. Finally, the hammer hits the strings with velocity $v_{i}$. The difference between $v_{e}$ and $v_{i}$, and the threshold on $v_{e}$ below which the hammer does not even touch the strings, are a nuisance for the musician. Although inevitable, this second phase also affects the aptitude to control nuances, so important in music. To quantify this notion, the control index $i_{c}=d v_{e} / d v_{i}$ is introduced, which can be easily calculated. Its limit is 1 for fortissimo but it approaches 0 for pianissimo. For the pianist, this corresponds to a steeply increasing difficulty to phrase music at low rather than at high dynamic levels. Differences between the grand and the upright piano will be discussed.
$5: 40$

2pMU11. An artificial neural network model for analysis and synthesis of pianists' performance styles. Roberto Bresin (Royal Inst. of Technol., Dept. of Speech, Music and Hearing, Drottning Kristinas v. 31, 10044 Stockholm, Sweden, roberto@ speech.kth.se)

A model for synthesis and analysis of professional pianists' performance styles by means of artificial neural networks (ANNs) is presented. The style of pianists was synthesized using Bruno Repp's measurements [J. Acoust. Soc. Am. 92, 2546-2568 (1992)] of Schumann's Trumerei as performed by 24 famous pianists. It will be shown that, by using significant score parameters, ANN can learn and reproduce the style of a pianist at a local level. The behavior of the trained ANN and the performance characteristics of the pianists were analyzed by running the trained ANN in a special way. All ANN input neurones were locked to fixed values, except one or two neurones whose input values were allowed to change in time. For example, if one of these neurones is related to the duration of the current note, the output of the ANN gives a curve showing expressive deviations produced by the ANN, when the duration of the note is varying. In this way it is possible to extract behavior rules of performing action of the ANN, and compare them with the performance style of pianists as well as of rule-based performance systems. The model is developed in the Java language and is available on the Internet.

\section{6:00}

2pMU12. Experience of students of composition and instrument construction at the National School of Music of UNAM in the auditory normalization of timbre with age and with exposure to different instruments. Eduardo Castro-Sierra (Natl. School of Music (ENM) of the Natl. Autonomous Univ. of Mexico (UNAM), Xicoténcatl No. 126, 04100 México, D.F., Mexico), Martha Gómez-Gama, Miguel Zenker (ENM, UNAM), and Santiago J. Pérez-Ruiz (Section of Acoust. of the Ctr. of Instruments of the Natl. Autonomous Univ. of Mexico)

A study of tonal memory using as stimuli synthesized harmonic sounds with a quasi-square-wave spectrum has been carried out at ENM. The subjects were young boys and girls (age span=8:1-15:6), starting their musical education at the Cycle for Musical Initiation, and young adults of either sex (age span=13:11-42), with experience of from 1 to 10 years in singing, piano, violin, clarinet, flute, trumpet, saxophone, guitar, percussion, or composition at the regular courses of ENM. While the children were adept in retaining in memory tonal changes of a major or a minor second using the synthesized stimuli, the older students showed much greater difficulty with this test, particularly those whose instrument had a timbre closer to that of the square wave (clarinet or saxophone), or who were undertaking studies of composition. Students of composition or instrument construction at the Instrument Construction Workshop of ENM have embarked on a further analysis of auditory normalization of timbres of diverse synthesized or natural sounds of instruments. It is expected that such an experience will allow the students to better conceptualize different timbre combinations as well as the timbre of individual musical instruments. 


\title{
Session 2pNSa
}

\author{
Noise: Railway Noise II \\ Markus Hecht, Cochair \\ Institut für Strassen- und Schienenverkehr, Fachgebiet Schienenfahrzeuge, Sekr. SG14, Salzufer 17-19, D-10587 Berlin, \\ Germany \\ Paul J. Remington, Cochair \\ BBN Systems and Technologies, 70 Fawcett, Cambridge, Massachusetts 02138, USA
}

Chair's Introduction-1:55

Invited Papers

2:00

2pNSa1. Today's railfreight noise situation. Markus Hecht (Institut für Straßen- und Schienenverkehr, Fachgebiet Schienenfahrzeuge, Sekr. SG 14, Salzufer 17-19, D-10587 Berlin, Germany)

In recent years railway freight wagons have tended toward a gradual increase in noise radiation. The reasons have been the transition from slide to roller-axle bearings, more welded joints instead of bolted or riveted ones, the increased number of four-axled wagons instead of two-axled ones, and higher medium speeds. Meanwhile, the competitors on the road and in the air have made substantial progress in noise reduction. The first major step toward quieter trains in railway freight was the decision of the German and the Swiss freight operators DB-Cargo and SBB-Cargo to replace cast iron brake blocks by composite brake blocks. This was done in the US decades ago, but the stopping distances in Europe are much shorter, about one-quarter of those in the US. Therefore, the braking forces are much higher, which results in higher thermal thrust on the running surfaces. A special wheel shape had to be developed to withstand this impact and to reduce the risk of broken wheels. Noise emission from European modern standard freight cars must be reduced by $25 \mathrm{~dB}$. Only then can the existing perception limits at densely used freight lines be reached at night. Measures to achieve this goal will be presented.

2:20

2pNSa2. Cause research and assessment of the acoustic behavior of rail vehicles using SFE AKUSRAIL. Hans Zimmer (SFE GmbH, Ackerstr. 71-76, D-13355 Berlin, Germany)

Many efforts have been made in the past to further develop knowledge of both the interior acoustics and sound emission of rail vehicles using numerical methods. One major advantage of numerical methods is the possibility of forecasting the acoustic behavior (without prototyping) and hence influencing the design of the vehicles, also in terms of acoustic criteria. Another advantage of this "virtual prototyping" is that it is possible to determine the contribution of individual components to the sound level within the frequency range analyzed, i.e., to analyze the causes of acoustic behavior. However, strict criteria must be met to satisfy the claim of a useable forecast of acoustic behavior. It must be possible to simulate important acoustic phenomena—such as structure/air coupling, absorption, reflection, emission, etc. The numerical model must be sensitive enough to permit the forecasting of trends. The interaction of rail and vehicle must be taken into consideration as a crucial precondition for identifying and analyzing acoustically critical frequencies. Another decisive criterion, however, is that it must be possible to assess not only the "actual" acoustic state, but also the underlying causes. This includes, for instance, knowledge of structure-borne sound paths and the structure and coupling situation.

2:40

2pNSa3. Roughness growth on tread-braked railway wheels: EURosabot theoretical studies. Anders Nilsson and Urmas Ross (MWL, Dept. of Vehicle Eng., KTH, S-100 44, Stockholm, Sweden, andersni@fkt.kth.se)

Rail and wheel surface irregularities are the main source of rolling noise. One of the main causes for wheel surface roughness is tread braking. Wheels braked by the most common tread brakes-cast iron blocks-have high roughness levels and, as usual, corrugation with wavelength of $30-100 \mathrm{~mm}$. The Brite Euram research project EUROSABOT deals with new solutions for tread brakes, which would lower the roughness level, prevent the buildup of periodic roughness, and consequently reduce the rolling noise, and at the same time would follow the safety requirements of railway operation. Field measurements, discussed in detail by Stefan Buehler in a companion presentation, in parallel with laboratory studies has distinguished that the interaction of thermo-elastic and vibrational instabilities is the main reason for corrugation generation. Computer simulations describing simultaneously thermodynamical, structural, and acoustic processes of the braking operation have proved to be unpractical for parametric studies and, instead, several simplified models are used. These models have shown that the main parameters affecting roughness growth are Young's modulus and coefficient of thermal expansion of the block material and damping of the system. 
2pNSa4. Eurosabot field measurements-Methods and results. Stefan Bühler (ABB Daimler-Benz Transportation Ltd., Zürcherstrasse 41, P.O. Box CH-8401, Winterthur, Switzerland)

Eurosabot is a European research project focused on rolling noise reduction of block braked railway vehicles. While braking, the interaction between block and wheel generates roughness patterns on the wheel which become the major excitation for rolling noise. The objective was to find a brake block (material and design) which combines the advantages of common cast-iron blocks with lower roughness generation without common drawbacks of other block families. Measurements of most relevant parameters were already available before Eurosabot, but boundary conditions and other parameters were different, inconsistent, or even unknown, which made it difficult to gain a reliable understanding of how roughness is generated. Therefore, Eurosabot's measuring campaigns were focused on strictly simultaneous monitoring of all relevant parameters under known and real boundary conditions. The following parameters were monitored over a long-term period in normal service: wheel and block wear, wheel roughness, braking distance, residual stress (wheel rim). During a concentrated campaign, rail roughness, rail impedance, rail accelerations, sound pressure, and sound power distribution were measured wayside, as well as sound pressure (on board), wheel and block accelerations, wheel and block temperature distribution, and friction coefficient on the vehicle. Results show that this method enables a complete judgment of the behavior of a block.

3:20

2pNSa5. Noise annoyance by freight trains. Anja H. M. Steenbekkers and Ronald G. de Jong (TNO Prevention and Health, P.O. Box 2215, 2301CE Leiden, The Netherlands, rg.dejong@pg.tno.nl)

In The Netherlands a new freight-only railway route, the "Betuweroute," is being planned to carry freight traffic from Rotterdam harbor east into Germany. Inhabitants of areas near the future route are concerned about noise and vibration, and question the relevance of current legislation for a "freight-only" line. Results of analyses on existing data show no consistent differences in exposure-response relations between routes with a small or high proportion of freight trains, once the effects of different noise levels are removed. Thus these results do not support the need for special legislative provisions for freight-only lines. However, hardly any freight-only train route was included in the analyses, and concern remains. The most western part of the future Betuweroute is an existing freight-only line, the "Harbour line." Some 109 inhabitants living near this line were interviewed in order to pretest the questionnaire for a social survey in areas along the future Betuweroute. For most homes accurate noise data are available. This offers another opportunity to test the hypothesis that, once the effects of different noise levels are removed, freight trains do not cause more annoyance than passenger trains. The results of this test will be presented.

\section{3:40-4:00 Break}

\section{Contributed Papers}

\section{4:00}

2pNSa6. Experiments and computations of noise reduction by barriers. Erik M. Salomons and A. A. F. M. Beeks (TNO Inst. of Appl. Phys., P.O. Box 155, 2600 AD Delft, The Netherlands)

Experimental and theoretical investigations are presented of the performance of noise barriers in the open air. The experimental work includes full-scale measurements on barriers and scale-model measurements on a barrier in a wind tunnel. The wind tunnel experiments indicate that meteorology has large effects on barrier attenuation. The results of the wind tunnel experiments are compared with results of numerical computations with the parabolic equation method. Good agreement with the experimental data is obtained only if wind speed profiles as measured in the wind tunnel are used in the computations. It is concluded that wind speed gradients induced by the barrier itself reduce the barrier attenuation considerably: reductions as large as $10 \mathrm{~dB}$ are found. The use of aerodynamically optimized barriers is proposed in order to minimize this reduction of barrier attenuation.

\section{4:20}

2pNSa7. Scale model experiments on new types of noise barriers. Djamel Ouis (Eng. Acoust., Lund Inst. of Technol., P.O. Box 118, S-22100, Lund, Sweden)

This paper is concerned with the results of experiments conducted on some new types of noise barriers. The experiments were conducted in the anechoic chamber at a scale model level and the barriers have different profiles with focus on the so-called forklike topped barrier. The quantity of interest is the sound-pressure level in the zone shadowed by the barrier. The arms of the barrier top were combined in several different ways and angles as well as also being mounted by either hard or soft cylinders to predict the effect of these latter on the edge diffraction phenomenon. The ground is taken either as hard on both sides of the barrier or a combination of hard and soft ground on, respectively, the source and receiver sides. [This work was supported by a grant from TeknikBrostiftelsen in Lund, which is gratefully acknowledged.]

\section{$4: 40$}

2pNSa8. Acoustical contribution analysis of railway wheels and tracks. C. Katzenschwanz (IABG GmbH, Einsteinstr. 20, 85521 Ottobrunn, Germany, katzenschwanz@iabg.de), G. Hölzl, L. Willenbrink, and T. Lölgen (Deutsch Bahn AG, D-80939 München, Germany)

In a numerical analysis the contribution of different railway wheels and the track on the sound power and the sound pressure was determined. Two different wheels under different loads and positions were compared. In a first step a unit mechanical excitation in the connection point between the wheel and the rail was used to analyze the structural displacements of each configuration with finite elements. The structural energy on the surface of the wheel and the rail was used to select critical frequencies using boundary elements. In a second step the sound pressure was displayed on visualization planes and the contributions of the wheel and the rail to the sound power and the pressure at different points were discussed for the critical frequencies. It could be shown that the main sound pressure was dominated by the wheel. The bending modes of the wheel contribute a lot to the sound pressure. For optimized wheels the bending stiffness should be therefore increased. The position of the wheel-over the sleeper or in between-did not influence the contribution very much as well as the loading of the wheel. 
calculated by approximating the normal velocity on the surface of the rail with the velocity produced by multipole sources within the rail. Their amplitudes are gained by minimizing the surface error by a method of least squares. The sound radiation is then the superposition of the pressure generated by all such multipole sources. It has been calculated considering one or two rails, coherent or not, each moving arbitrarily in the $x$ and $y$ directions (perpendicular to the length of the rail), with or without frequency-dependent ground absorption. The results of the approach are demonstrated.

\section{$5: 40$}

2pNSa11. Vibration assessment in buildings from the underground trains. Illya E. Tsukernikov, Samuel A. Rybak, Stanislav A. Kostarev, and Igor A. Nekrasov (Lab. of Acoust. and Vib., Tunnel Assoc., 21 Sadovaya-Spasskaya St., Moscow, 107217, Russia)

The method approved by the Moscow government in 1997, which has been implemented by the regulation "Guideline for assessment of vibration from underground train operation in dwellings and administrative buildings"' [1998, Moscow, TIMR], is considered. The method is based on the control of nonsteady vibration by two normative parameters: maximum and equivalent values of rms acceleration, and assessment is made as for frequency-weighted quantities, and so in octave frequency bands at the range $22,5-90 \mathrm{~Hz}$. The method makes it possible to determine the values of maximum effective rms acceleration during the rating time stated. The procedure of equivalent rms acceleration calculation is analogous to one of DIN 4150, part 2, and the train operation schedules during the rating times are used. Results of testing the method at one of the Moscow Metro lines are discussed. Recommendation on the enlargement of the frequency range controlled and on the correction of the criterion of the frequencyweighted rms acceleration assessments are proposed.

2pNSa10. Calculation of the three-dimensional sound radiation of rails using the multipole approach. Berndt $\mathrm{W}$. Zeitler and Michael Moeser (Institut fuer Technische Akustik, Einsteinufer 25, 10587 Berlin, Germany, zeitler@mach.ut.tu-berlin.de)

Railway noise is of concern where an important factor is the sound radiated from the rail. This has been studied by using a multipole approach. The three-dimensional sound radiation of a vibrating rail can be

\section{Contributed Poster}

This poster will be on display in the Poster Gallery from Monday to Wednesday, 15-17 March. The author will be at the poster from 10:00 a.m. to 12:00 noon on Tuesday, 16 March.

2pNSa12. Prediction of railway-induced vibration by means of transfer functions. Juergen Peters and Gerhard Prestele (Moehler + Partner, Schwanthaler Str. 79, D-80336 Muenchen, Germany)

The prediction of train-induced vibrations in urban areas has to meet many, partly contradictory aspects. Accuracy of predicted vibration with respect to disturbance of residents and measures of reduction of groundborne vibration, unknown conditions of transmission, large number of buildings to investigate, economical and time aspects also have to be taken into account. The extension of an existing above-ground railway into two tracks in dense developement was taken to test a prediction method based on measured transfer functions characterizing the new track. These investigations were done at an intermediate stage of construction without gravel and sleepers. The transfer functions are used to calculate emission spectra based on a known set of train spectra and corresponding transfer functions investigated elsewhere. The train spectra have to match the super/ substructure of the new track and characteristics of the train to be predicted (e.g., velocity). Recent results are discussed. 


\title{
Session 2pNSb
}

\author{
Noise: Noise at the Workplace I \\ Alice H. Suter, Cochair \\ Alice Suter \& Associates, 575 Dogwood Way, Ashland, Oregon 97520, USA \\ Gustav A. Sehrndt, Cochair \\ Consultant, Kapuzinerstrasse 8, D-50737 Cologne, Germany \\ Chair's Introduction-1:55 \\ Invited Papers
}

\begin{abstract}
2:00
2pNSb1. The last 100 years of magazine articles on noise. Robert D. Bruce (Collaboration in Science and Technology, Inc., 15835 Park Ten Pl., Ste. 105, Houston, TX 77084)

Articles on "noise" that were referenced in the Reader's Guide to Literature (and similar guides) from 1890 to 1990 were reviewed recently. These articles present an interesting history of the spread of knowledge about noise-induced hearing loss. By determining the readership of each magazine and assuming that the information in that magazine was representative of the knowledge of that readership, it is possible to attribute certain levels of knowledge chronologically about noise-induced hearing loss to different segments of society. For example, the first group to learn about noise-induced hearing loss consisted of doctors who directly diagnosed hearing losses. Next to learn were the doctors who read journal articles written by the first doctors. Soon after the medical articles were published, the information propagated to the scientific/engineering and financial communities, and they added their own particular insights on the subject. Considerably later, magazines read by workers and their families published information on noiseinduced hearing loss, but seldom with sufficient information to enable workers to protect their own hearing.
\end{abstract}

2:20

2pNSb2. Combined effects of noise and whole body vibration in the working environment. Marianne Schust and Helmut Seidel (Federal Inst. for Occupational Safety and Health, Office Berlin, Nöldnerstr. 40-42, 10317 Berlin, Germany, arbeitsmedizin@basam.b.shuttle.de)

The simultaneous exposure to noise and whole-body vibration (WBV) is the most frequent combination of physical factors at workplaces. Both factors probably act not-independently of each other. The results of the studies regarding the aural effects indicate that additional exposure to WBV amplifies the temporary threshold shift as well as of the permanent threshold shift. Various parameters, i.e., postural sway, vision, and cardiovascular changes, were investigated in experimental studies to clarify the extraaural physiological effects. The results demonstrate a dependence on the intensity and the frequency spectrum of the noise as well as of the WBV. Mutually amplifying interactions of noise and WBV were observed. The events of experimental research regarding the subjective assessment of exposure conditions suggest the following conclusions: an increase of noise intensity causes stronger sensations of the WBV intensity except for WBV with main frequencies around $2.5 \mathrm{~Hz}$. Changes of WBV intensity have no effect on the subjectively experienced loudness and noise-related annoyance. Compound judgments of the entire situation are influenced by an interaction of noise and WBV. The results are discussed to derive proposals for guidelines considering combined effects.

\section{2:40}

2pNSb3. Hearing disorders from combined exposures to noise and solvents: A review. Thais C. Morata (Natl. Inst. for Occupational Safety and Health, 4676 Columbia Pkwy./C 27, Cincinnati, OH 45226, thaiscm@aol.com)

Not until recently was solvent exposure found to have a significantly adverse effect on hearing. In some cases the risk can be comparable to the most potent and best-known occupational factor, excessive noise. In animals, combined exposure to solvents and noise can result in an increased loss of auditory function beyond the simple addition of the effects of each agent. An increased prevalence of hearing loss has been reported in humans as well. The balance function can also be affected by certain industrial chemicals. The importance of these observations becomes clear when the magnitude of exposed populations and the number of potentially hazardous chemicals found in work environments are taken into account. The need for research in this area is further heightened by the fact that there are no guidelines or standards for combined exposures to chemical and physical agents. The present paper reviews the effects of combined exposures to solvents and noise on hearing function and examines study designs, hearing assessment alternatives, and strategies for the analysis of these effects. 
2pNSb4. Communication and warning signal perception. Judy Edworthy (Dept. of Psych., Univ. of Plymouth, Drake Circus, Plymouth, Devon PL4 8AA, UK, jedworthy@plymouth.ac.uk)

Warning signals are used throughout industry and commerce, and often provide vital sources of information. Due to developments in digital technology, it is now possible to deign any sort of auditory signal, some of which may be effective, some of which may not. In this paper a methodology is outlined which takes a step-by-step, user-centered approach to the design and implementation of warning signals in noisy environments with the aim of creating warning signals which are both easy to understand and to act upon. It begins with the selection of referents (the events to be signaled), through an iterative design process which involves tests of appropriateness, learning and confusion, urgency, and recognition, to operational testing which includes adaptation to the noise environment, consideration of the complete warning set, and so on. The methodology is based on a standardized procedure for designing and implementing public information systems [ISO 7001, "Specification for public information systems" (1979)]. The paper describes the methodology in detail.

3:20

2pNSb5. Noise exposure, hearing loss, and hearing conservation in the construction industry. Alice H. Suter (Alice Suter \& Assoc., 575 Dogwood Way, Ashland, OR 97520)

Workers in certain sectors of the construction industry incur more noise-induced hearing loss than in most other trades. This paper reviews data on noise exposure and hearing loss in the construction industry, gathered mainly in Washington State, British Columbia, Canada, and Sweden. The problem of worker mobility, where temporary employment is typical even for unionized workers, has hampered the implementation of effective hearing conservation programs in the construction industry. Solutions to this problem include a central repository for audiometric test results and information on training and noise control programs. Multiemployer benefit programs concerning health insurance and pensions provide models in the United States. A hearing conservation program specific to construction noise has been successfully implemented in British Columbia, with a resulting decrease in the incidence of noise-induced hearing loss.

\section{3:40}

2pNSb6. Assessment and remediation of perceived handicap in subjects with acquired hearing impairment. Lillemor R-M. Hallberg (Nordic School of Public Health, Box 121 33, 42042 Göteborg, Sweden, Lillemor.Hallberg@nhv.se)

In a treatment-comparison group design, a group-rehabilitation program developed for hearing-impaired individuals and their close relatives was evaluated. The majority of subjects had hearing loss from noise exposure. The aim of the program was to give adequate information, psychosocial support, and training for effective coping strategies. Sixty-six consecutive patients at the hearing clinic were included in a pilot study. Standardized questionnaires, intended to measure "perceived handicap," "social support," "perceived negative attitudes," "acceptance of hearing loss," and "communication strategies" (maladaptive behavior, verbal strategies, and nonverbal strategies), were used at the end of the last group session (short-term evaluation) and with postal questionnaires four months later (long-term evaluation). The evaluation showed that the use of maladaptive behavior (e.g., guessing, pretending to hear, and avoiding interactions) was significantly decreased in the treatment group in a short-term as well as in a long-term perspective ( $p=0.000$ and $p=0.032$, respectively). The use of verbal strategies was significantly increased in the treatment group after the rehabilitation $(p=0.020)$, but this increase did not reach a significant level four months later. Self-reported handicap was decreased in some conditions. Presently, data from ongoing rehabilitation groups are assessed and statistical data analysis will follow.

\section{4:00-4:20 Break}

\section{4:20}

2pNSb7. Noise control case studies. Keith A. Broughton (Health and Safety Executive, Magdalen House, Bootle, Merseyside L20 3QZ, UK)

Giving advice to owners and employers on how they can introduce methods of controling noise in industrial situations is often difficult. Many years ago the noise specialist inspectors built up a database of successful control methods they witnessed in their normal duties in visiting factories. These were collated into a case study book and sold. Recently this exercise was repeated and the second book, Sound Solutions, was issued. Aimed specifically at employers, it gives examples from a wide range of industries of noise problems which have been successfully overcome. It outlines the original problem, gives the solution, and indicates an approximate cost, and gives advice on where such control can be obtained. In the introduction advice is given so that the employer can ask a consultant or engineer questions on the method to be employed to solve a problem, so that they have a better understanding of the control process involved. The guidance is published by the HSE, a department of government.

2pNSb8. Noise declaration for hand-held machinery. Ilkka Kyttälä (Ministry of Social Affairs and Health, Dept. for Occupational Safety and Health, Box 536, FIN-33101 Tampere, Finland) and Erkko P. Airo (Inst. of Occupational Health, FIN-00370 Helsinki, Finland)

A study on noise declarations and safety of hand-held machinery was executed in Finland in 1995-1996. Acceptable declarations were available for $77 \%$ of pneumatic and $94 \%$ of combustion-type machinery but only for $50 \%$ of electric machinery. This problem of machinery not having noise declaration can be corrected by national or international market control operations. For example, such a project on pneumatic hand-held machinery is going on in Finland by the occupational safety and health administration. The results will be presented at the beginning of 1999 . Another problem is that the available noise declarations were usually correct but not 
necessarily valid. Laboratory measurements showed that declared values were correct, but the sound-pressure levels at the operator's position were higher in practical use. For example, angle grinders' declared idling SPLs were 80-86 dB, in a workshop they were 90-95 dB, and during metal processing the SPL was $110 \mathrm{~dB}$. The reason for this is obvious: the quiet simulated, standardized operating conditions produce less noise than the typical use. The purpose of the system, the possibility to choose a low-noise machine, is partially lost. Too low declared values may also lead to false conclusions about the need of hearing protection.

\section{Contributed Papers}

\section{5:00}

2pNSb9. Common problems in noise control projects. Sergio Beristain (Mexican Inst. of Acoust. Lab., Faculty of Elec. and Mech. Eng., Natl. Polytechnic Inst., P.O. Box 75805, 07300 Mexico City, Mexico)

In actual industrial noise control, it is common to face obstacles before being able to analyze the noise, in addition to others arising in the process of analysis and designing the proper solution: usually it is not a new installation, which produces big savings in the future, but noise due to bad installations or irregular growth of industrial plants. This paper discusses the most common problems found in practice, and presents some ideas for facing some of them. Among them are economics, legislation, lack of knowledge or interest, lack of trained personnel for making the installation, etc., and the noise control project itself, which indeed is very important. This means that the noise control specialist should be ready to face conditions fully outside his or her expertise, but completely related to the problem to be solved. There are some additional aspects not related with technique, standards, or economics, which are opposed to the implementation of the designed solution, like culture, education or "the expectation that nobody will get deaf in the future if they did not get deaf today." Knowledge of all of the above limitations is essential to avoid failure in noise control in new or rebuilt installations or in hearing conservation programs.

\section{$5: 20$}

2pNSb10. Polish system of workers' protection against noise. Krystyna Pawlas (Inst. of Occupational Medicine and Environ. Health, 13 Koscielna str., 41-200 Sosnowie, Poland, kape@imp.sosnowie.pl)

In Poland, like in other industrial countries, the protection of workers' health against hazards of occupational environment is a subject of many regulations. The basic act is the Code of Labor. Executive regulations connected with the Code are edited by the Ministry of Health and Social Welfare and the Ministry of Labor and Social Affairs. The Ministry of Health controls the organization of occupational health services, e.g., requirements for persons performing health examinations, principles of hearing examination, assessment of hearing disability, and the principle of certification of the hearing damage as occupational disease as well as monitoring of noise at workplaces. The Ministry of Labor establishes maximum allowable levels, organizes safety and hygiene services, and sets the principles of education in the field of occupational hygiene. The Ministry of Labor recommends standards for measuring and assessing noise at workplaces. These regulations are the basis for the hearing conservation programs. Polish legislation has been adjusted recently to har- monize with the currently standing legislation in the European Community. The main principles at present are the same as those accepted by the EC, but there are some differences concerning details of legislation and the organization of medical as well as occupational hygiene services.

\section{$5: 40$}

2pNSb11. A DRC-based analysis of the auditory hazard from exposure to impact noises generated by carpentry tools and tasks. Robert M. Lilkendey (Jaffe Holden Scarbrough Acoustics, Inc., 114A Washington St., Norwalk, CT 06854, rlilkendey@jhsacoustics.com)

To assess the risk of hearing from impulsive sounds several countries have developed damage risk criteria (DRC) which establish limits for an exposure to impulse noise based on key parameters of the impulse and a threshold for acceptable hearing loss. The German, French, Dutch, and an adjusted CHABA DRC were used to assess the potential for carpentrybased impact noises to present an auditory hazard to construction workers under normal working conditions. Measurements of impact noise events from compressed air nailguns and common hammering tasks were made in the field and simulated in a laboratory. The peak sound-pressure level, duration, and A-weighted energy of each impact were determined by digitizing the event and then processing the waveform using two impulse noise analysis programs. The maximum number of allowable exposures was calculated for each event based on the levels set by the DRCs and compared to published and observed productivity rates. The results showed that among the four DRCs, the German was the least protective, finding only 5 of the 12 exposures to be hazardous, and the French (7), Dutch (11), and adjusted CHABA (12) incrementally more protective.

\section{6:00}

2pNSb12. Auditory damage due to impulses or continuous noise. Gerald Fleischer (Div. of Auditory Res., Univ. of Giessen School of Medicine, D-35392 Giessen, Germany)

In order to determine the detrimental effect of continuous noise versus impulsive noise, a number of groups were examined: school children, university students, soldiers, workers, pilots, and amateur and professional musicians. Auditory performance was determined by means of pure tone audiometry up to $16 \mathrm{kHz}$. Auditory load throughout life was estimated, using a detailed questionnaire that included the aspects of profession, hobbies, sports, and consumption of music. Occurrence and severity of tinnitus were also determined. It can be shown that impulsive noise is more damaging to the ear than continuous noise of the same $L_{\text {eq }}$. Analyzing the situation at the workplace, it can be concluded that the damaging effects of acoustic impulses are not properly measured by the $L_{\mathrm{eq}}$. 


\title{
Session 2pNSc
}

\section{Noise and Physical Acoustics: Experience with Noise Prediction I}

\author{
Keith Attenborough, Cochair \\ Faculty of Technology, The Open University, Milton Keynes MK7 6AA, UK \\ Karl-Wilhelm Hirsch, Cochair \\ Institut für Lärmschutz, Arnheimer Strasse 107, 40489 Düsseldorf, Germany
}

Invited Papers

2:00

2pNSc1. Remarks on acoustical descriptors for explosions in air. Karl-Wilhelm Hirsch (Inst. fuer Laermschutz, Duesseldorf, Germany)

Currently, ISO/TC43/SC1/WG51 is collecting "experiences" with measuring and predicting shooting noise. One goal of this working group is to write a guideline for the measurement of source data of shooting noise, taking into account the special characteristics of these high energy, impulsive blasts. There are three special aspects with shooting noise: First, there are nonlinear effects close to the source. Second, the strong directivity of blast sources plays a very important role. Third, in contrast to most of the continuous noise sources, blasts are single events which involve coherence effects during source and receiver measurements. Therefore, a dedicated set of emission data has to be introduced to describe a blast source with respect to sound propagation models. The presentation will discuss the definition of a set of appropriate source descriptors for muzzle blasts. The source will be treated as a linear spherical source with directivity. Its intensity will be defined in the far field and its directivity pattern will describe the acoustical strength. The radius of this artificial source depends on the blast energy and is an important parameter when determining the appropriate measurement distance for source measurements.

2:20

2pNSc2. Measurements of acoustical emissions from large-calibre weapons. Christa Korff (Lärm-Meßstelle 1 Bw, WTD 91, D-49716 Meppen, Postfach 1764, Germany)

Extreme pressure waves and a wide frequency band characterize the environment of a firing large-calibre weapon. Here the usual way to gain acoustical source data therefore fails. For this reason an international working group has developed a standard procedure to obtain the sound emission from such weapons. This will be described. The German Ministry of Defense adopted the method to collect the basic source data for a computer model to calculate the emissions of noise caused by the shooting artillery and explosions in the vicinity of military training areas. It supported the experiments that have been carried out in the last years at the Meppen proving ground in northern Germany. There the environmental conditions defined by the working group are met rather well. First experiences with the application of the standard procedure and some results of the model calculations are reported.

2:40

2pNSc3. Source data for small arms noise. Larry L. Pater (U.S. Army Construction Eng. Res. Labs., Champaign, IL)

Small arms source data are required for calculation of far-field noise levels. Advantages and disadvantages of various methods of obtaining source data for small arms noise are discussed, and specific examples are presented. Important issues include nonlinear effects, directivity of muzzle blast and projectile shock noise fields, isobar shapes, wavefront shapes, and blast wave temporal characteristics, particularly shape and duration. A methodology is presented for obtaining source data that is free of inaccuracies due to ground reflections and ground impedance effects. Source data have been measured for a number of small weapons, both rifles and pistols. Example data are presented as waveforms, peak levels, overall sound exposure levels, and spectral distribution of 1/3-octaveband sound exposure levels. Data are presented separately for muzzle blast and projectile shock, and special attention is paid to the effect of interactions of the blast noise sound wave with the ground. [Work supported by U.S. Army.]

\section{3:00}

2pNSc4. Results from Norwegian sound propagation experiments. Lars R. Hole (Norwegian Defence Construction Service (NDCS), Oslo mil/Akershus, 0015 Oslo, Norway, larsr@gfi.uib.no)

This paper describes four sound propagation experiments that were undertaken in Norway from 1994 to 1996. Trials were carried out at Finnskogen; a long-range site, and at Haslemoen; a short-range site. Summer and winter conditions were investigated at both sites. Two similar experiments were carried out at each site with and without snow cover. At Finnskogen, an experimental array of 24 by $24 \mathrm{~km}$ was set up in a terrain with smooth hills, primarily covered by forest. At Haslemoen, propagation was investigated out to $1.5 \mathrm{~km}$ above a uniform forest and a uniform, flat open field of pasture land. One, 8 , and $64 \mathrm{~kg}$ cylindrical charges of C-4 explosives 
were used as sound sources. Measurements of acoustics, seismic ground response, complex ground impedance, and meteorology were carried out simultaneously. This paper gives an overview of the experiments and presents examples of data. An overview of theoretical research based on the collected dataset is also given. Literature for further details is referred. An easily accessible database with all data is available. Detailed information is also given at www.bpof.inter.net.

3:20

2pNSc5. Measured and computed effect of meteorological condition and ground surface on propagation of impulsive sound to 20 km. Michael J. White (Appl. Res. Assoc., 5941 S. Middlefield Rd., Littleton, CO 80123)

The cumulative propagation effects of weather and ground surface on sound exposure levels at long distances from an impulsive sound source are not well established. Measurements of sound exposure and peak pressure at distances up to about $20 \mathrm{~km}$ from explosions were made by the U.S. Army for three types of ground conditions. These data, along with measured weather profiles and ground impedance parameters, were used as inputs to the FFP model, for comparison of measurement to prediction. The 1/3-octave band attenuations were calculated for band center frequencies from $1 \mathrm{~Hz}$ to $1 \mathrm{kHz}$, for the measurement microphone locations in the study. A great deal of the variation in signal level from event to event was predicted by the FFP model, without resort to nonlinear propagation effects or to turbulent scattering. Groups of cases with similar signal attenuation with distance and similar weather profiles may provide a reasonable basis for classifying long-distance propagation conditions. Such a classification would have merit as an efficient way to assess long-term noise averages or to estimate the error in short-term predictions of noise levels. [Work supported by USACERL and AMSAA.]

3:40

2pNSc6. Blast noise propagation above a snow cover at Haslemoen, Norway. Donald G. Albert (USACRREL, 72 Lyme Rd., Hanover, NH 03755) and Lars R. Hole (Norwegian Defence Construction Service, Environ. Section, Oslo mil/Akerhus, 0015 Oslo, Norway)

During the short-range winter measurements at Haslemoen, Norway, a series of blast wave forms produced by 1-kg charges of $\mathrm{C} 4$ explosive fired $2 \mathrm{~m}$ above a dry snow cover 11 to $20 \mathrm{~cm}$ thick were recorded after propagating horizontally 100 to $1400 \mathrm{~m}$. In attempting to model these experiments, it was found that the usual viscoelastic treatment of the ground was unable to match the recorded wave forms. A rigid-porous model of the snow cover was required to obtain good agreement. The measured wave forms, with a peak frequency around $50 \mathrm{~Hz}$, show pulse broadening effects similar to those previously seen in pistol shot experiments using higher frequency waves over shorter propagation distances. The modeling predicts an average snow cover depth of $15 \mathrm{~cm}$ and effective flow resistivities of $30-41 \mathrm{kPa} \mathrm{s} \mathrm{m}^{-2}$, in agreement with snow pit observations and with previous acoustic measurements over snow. For propagation in the upwind direction, the pulse broadening caused by the snow cover interaction is lessened, but the overall amplitude decay is greater because of refraction of the blast waves.

$$
\text { 4:00-4:20 Break }
$$

\section{4:20}

2pNSc7. Air-ground interaction effects in the propagation of low-frequency impulse noise. Christian Madshus, Amir M. Kaynia (Norwegian Geotechnical Inst., Anal. Div., P.O. Box 3930, Ullevaal Hageby, N-0806 Oslo, Norway, cm@ngi.no), and Lars R. Hole (Norwegian Defence Construction Service, N-0015 Oslo, Norway)

Four large-scale sound propagation experiments performed in Norway from 1994 to 1996 under both summer and winter conditions clearly demonstrate that air-ground interaction has a significant effect on the propagation of low-frequency impulse noise. During the experiments, specific measurements were made for detailed investigation of this interaction. The paper will present the instrumentation used, and some typical measured data. Various numerical simulations of the experiments are made, covering both poro-elastic and visco-elastic description of the ground. Based on the measured data, theoretical considerations, and the numerical simulations, the paper will discuss mechanisms for acoustic energy loss from the air into the ground. Special emphasis will be put on the effect of the interaction of the propagating air pressure with the slow p-waves and with the dispersive Rayleigh waves in the ground. The relative importance of these two interaction mechanisms on the energy loss and thus on the sound attenuation will be examined for various ground conditions.

4:40

2pNSc8. A comparison of engineering methods for predicting ground effect. Keith Attenborough (Faculty of Technol., The Open Univ., Milton Keynes MK7 6AA, England)

Methods for calculating ground effect for broadband noise are reviewed and predictions of these methods are compared with recent data for propagation from a fixed jet engine and measurements over farmland in various states of cultivation. The review includes empirical, standard, and approximate analytical methods. The comparison leads to a discussion and evaluation of their relative merits. [Work supported by EPSRC and BBSRC (UK).]

5:00

2pNSc9. On the applicability of models for outdoor sound. Karsten B. Rasmussen (Dept. of Acoust. Technol., Bldg. 352, Tech. Univ. of Denmark, DK-2800 Lyngby, Denmark, kbr@dat.dtu.dk)

The suitable prediction model for outdoor sound fields depends on the situation and the application. Computationally intensive methods such as parabolic equation methods, FFP methods, and boundary element methods all have advantages in certain situations. These approaches are accurate and predict not only sound pressure levels but also phase information. Such methods are, however, not 
always able to predict the sound field for more complicated scenarios involving terrain features, atmospheric wind and temperature gradients, and turbulence. Another class of methods is based upon approximate theory - such methods are inherently less accurate but generally faster in computation and may also provide results for complicated scenarios. The accuracy of different approaches in realistic situations is discussed based upon measured and simulated data.

\section{5:20}

2pNSc10. Standardization efforts in Germany on the quality of software products for the prediction of environmental sound emission. Ulrich J. Kurze (Müller-BBM GmbH, Robert-Koch-Str. 11, D-81252 Planegg, Germany)

Based on ISO/IEC 12119 for quality requirements and testing of software packages, a special standard has been drafted for software products designed for noise mapping and related tasks of environmental noise control. The most important aspects are related to the definition and testing of software precision and to an interface suitable for data exchange between various programs. In a first step, the potential consistency of the software calculation procedures with standarized procedures is checked by test cases. Since switches can be set in most programs to accelerate calculation procedures for complex and extended situations, a second step is required to control the precision obtainable by the user for his special application. This is done on a statistical basis. Most of the effort is generally spent with the collection, adjustment, and input of geometrical data. In order to simplify repeated runs for modified situations and to allow for maximum flexibility of users, a common interface has been defined for all acoustically relevant data determined for a first run. This helps avoid errors and allows for data exchange suitable for comparison of different programs and situations.

2pNSc11. Aircraft noise model corrected by operational and propagation effects. Oleksander I. Zaporozhets, Vadim I. Tokarev (Acoust. Lab., Kyiv Intl. Univ. of Civil Aviation, 1, Ave. Cosmonaut Komarov, Kyiv, 252058 Ukraine, zap@elan-ua.net), Keith Attenborough, and Kai M. Li (The Open Univ., Milton Keynes MK7 6AA, UK)

Although there are mathematical models for predicting aircraft noise, the requirements on their accuracy have recently increased. Possibilities for model improvements are reviewed with respect to both noise source generation characteristics and noise propagation effects. The review indicates that the required corrections are not standard since they depend on the physical model of the noise source. The results of aircraft noise assessment for different types of aircraft and for different models of noise propagation are discussed. Recommendations for their use in computational schemes are made. The most rational method generates the required corrections for each type of aircraft according to the operational and noise propagation conditions in a given situation.

6:00

2pNSc12. FLULA2: Aircraft noise prediction and measurements. Georg Thomann and Rudolf Buetikofer (EMPA, CH-8600 Duebendorf, Switzerland)

Aircraft noise predictions of the program FLULA2 are compared with airport noise monitor measurements and with single flight events. Further, the versatility of using a point source model for simulation is discussed and the implications of accurate noise predictions on land use planning are outlined. The aircraft noise simulation program FLULA2, developed at EMPA, uses a point source with a directivity characteristic in contrast to many other models which use line segments and correction terms. With the powerful model of a moving point source, the level-time history of a single flight can be reconstructed. Thus all acoustic effects (topography, spectral air absorption, ground effects, ... ) may be included and any noise metrics can be calculated. The program is validated by comparing single events (the level-time history of measurements and of calculations) and by comparing the yearly average of measurements at monitoring stations around airports with the $L_{\mathrm{eq}}$ predictions. There are variations, but there is no systematic under- or overestimation of the noise calculations compared with the monitors. 


\title{
Session 2pNSd
}

\section{Noise: Computational Aeroacoustics II: Methodology and Applications}

\author{
Sanjiva K. Lele, Cochair \\ Department of Aeronautics and Astronautics, Stanford University, Stanford, California 94305-4035, USA \\ Christophe Bailly, Cochair \\ Laboratoire de Mécanique des Fluides et d'Acoustique, Ecole Centrale de Lyon \& UMR CNRS 5509, BP 163, 69131 Ecully, \\ Cedex, France \\ Invited Papers
}

2:00

2pNSd1. Aeroacoustic simulations and stochastic approach using linearized Euler's equations. Christophe Bailly and Daniel Juvé (Lab. de Mécanique des Fluides et d'Acoust., Ecole Centrale de Lyon, France and UMR CNRS 5509 BP 163, 69131 Ecully Cedex, France)

Sound generation and propagation in a turbulent flow is a very difficult numerical problem. Indeed, acoustic fluctuations are very small by comparison to the mean and turbulent fields. Linearized Euler's equations provide accurate numerical solutions only in working on perturbations. In the stochastic noise generation and radiation model, the mean flow is calculated by solving the Reynoldsaveraged Navier-Stokes equations. Then, Euler's equations are linearized around this mean flow field. A turbulent source term is introduced in the equations, and the turbulent velocity field is modeled by a sum of random Fourier modes. The numerical solution is obtained by using a dispersion-relation-preserving scheme in space, combined with a fourth-order Runge-Kutta algorithm in time. In order to validate the numerical method, radiation of multipolar sources in a uniform and sheared mean flow has been investigated in a two-dimensional case. The calculated solutions favorably compare with analytical solutions or with ray tracing. Then, the SNGR model is applied to a subsonic jet noise in a 3-D geometry. Numerical results and future developments will be discussed.

2:30

2pNSd2. CAA activities at ONERA using LES. Eric Manoha, Bruno Troff, Christelle Seror, Gilles Rahier, and Pierre Sagaut (Office Natl. d'Etudes et de Recherches Aerostatiales (ONERA), Dept. of Computational Fluid Dynam. and Aeroacoustics, BP 72 , 92322 Chatillon Cedex, France)

Most CAA applications rely on hybrid methods providing the radiated sound (using the Lighthill analogy, Kirchhoff integral or linearized Euler equations) from unsteady CFD. In that context, LES is widely considered as a compromise between full DNS and unsteady RANS. For two years, ONERA has undertaken studies aiming at understanding and applying LES in CAA. Former works focused on coupling LES to the Lighthill analogy. One typical application was the prediction of the noise generated by vortex shedding at the trailing edge of a thick flat plate. The incompressible LES was performed on a Cartesian mesh using the ONERA "PEGASE" code. The far-field noise was computed using the Curle formulation and favorably compared to airfoil noise experiments. More recently, a theoretical study analyzed the contribution of the LES subgrid scale model, or cutoff wave number, to the noise production computed from the Lighthill tensor, concluding that modeling this contribution may be important to correctly estimating the acoustic field. Other CAA applications of LES are now under progress: flap side-edge noise from high-lift devices of landing aircraft, and jet noise, with comparison of the Kirchhoff integral and Lighthill analogy. Coupling LES with linearized Euler equations is also a topic of interest.

\section{3:00}

2pNSd3. Low dispersion schemes. Mathematical model and demonstrative cases. Oktay Baysal (Aerosp. Eng. Dept., Old Dominion Univ., Norfolk, VA 23529-0247)

Considering the computational efficiency along with the high-fidelity to the underlying physical principles, significant advances have been achieved in aeroacoustics simulations. This, however, appears to have remained somewhat limited to the modeled periodic noise generation and propagation by assuming linear waves in uniform flow. Toward this goal, some useful applications have been computed with low-dispersion schemes, by solving the linearized Euler and Navier-Stokes equations. In addition to the benchmark cases reported in the CAA Workshops, these included a supersonic jet noise simulation. On the other hand, there are numerous aeroacoustics applications, such as subsonic jet noise and cavity noise, where the linear wave and uniform flow assumptions would be too compromising. Consequently, the linear dispersion-relation-preserving scheme and its boundary conditions have been extended to the nonlinear equations. It has been tested for a number of simple initial-value and periodic-source problems. Presently, a cavity noise problem and its suppression are being computed with this computational model. [Work partially supported by NASA Langley Research Center.] 
2pNSd4. Direct computation of sound generation and radiation from a "Singing Wire" (or direct computation of Aeolian tone). Duck Joo Lee and Jae Wook Kim (Aerosp. Eng. Dept., Korea Adv. Inst. of Sci. and Technol. (KAIST), 373-1 Kusong-dong, Yusong-gu, Taejon, Korea 305-701)

Sound from a singing wire, known as "Aeolian Tone," is simulated by directly solving unsteady compressible Navier-Stokes equations. The surface pressure on the surface of the wire in the presence of wind fluctuates periodically and the pressure near the surface radiates as a "dipole sound." The mechanisms of the surface pressure fluctuation are closely related to vortex shedding from the wire. An OHOC (optimized high-order compact) scheme is used to have high resolutions of the flow and the sound. A new formalism of characteristic boundary conditions in the body-fitted generalized coordinates is presented. Extended kinds of numerical boundary conditions for simulating subsonic inflow, nonreflecting outflow, and solid wall are formulated on the basis of characteristics and applied to computational aeroacoustics (CAA). The boundary conditions are applied to actual computation of 2-D wire having a cylindrical cross section on nonuniform and nonrectangular grid meshes. Far-field acoustic pressures due to Karman vortex streets are measured and the Strouhal number is evaluated correctly. The feasibility of using the generalized characteristic boundary conditions is investigated for sound generation owing to an unsteady flow around a body.

4:00-4:20 Break

2pNSd5. Acoustic radiation due to gust-airfoil and blade-vortex interactions. Ramesh K. Agarwal (Wichita State Univ., Natl. Inst. for Aviation Res., Wichita, KS 67260-0093)

The problem of acoustic radiation due to gust-airfoil and blade-vortex interaction in compressible mean flow is considered numerically. The acoustic perturbation field is calculated by solving the acoustic equations derived from the unsteady Euler equations by linearizing about a steady mean flow and assuming a single frequency disturbance. A computational code is developed which is validated by computing the unsteady loads on a flat plate due to a gust in incompressible flow (Sears problem), and unsteady loads and acoustic radiation due to a compact and noncompact gust in compressible flow. For these three cases the numerical results are compared with the exact analytical solutions and asymptotic solutions. Excellent agreement is obtained. The validated code is then employed to perform parametric studies by varying the Mach number of the mean flow, the angle of attack, and the geometry (thickness and camber) of the airfoil. Conclusions are drawn about the magnitude and phase of acoustic radiation due to gust-airfoil interaction for various Mach numbers, angles of attack, and airfoil geometries. The validated code is also employed to compute the acoustic radiation due to blade-vortex interaction. Calculations are compared with those of other investigations for both the mean flow and the acoustic variables.

\section{4:50}

2pNSd6. On the coupling of the inverse problem with computational aeroacoustics. Xiadong Li (Dept. of Jet Propulsion, No. 407, Beijing Univ. of Aeronautics and Astronautics, Beijing 100083, PROC)

This presentation is to survey the recent developments and advances of inverse problems arising from various aeroacoustic applications, with the emphasis on the inverse problem of the Ffowcs Williams and Hawkings (FW-H) equation. Generally an inversion physical model should be first established to relate the sound source with its radiated acoustic field. The main difficultly is that most inverse problems are mathematically ill posed. The singular value decomposition (SVD) technique combined with the Tikhonov regularization method has shown to be a very powerful tool. Also, the generalized cross-validation (GCV) and L-curve criterions are rather suitable for choosing an optimal regularization parameter. Several studies have shown that the aeroacoustic source strength distributions can be reconstructed using the radiated acoustic field data, such as a propeller [X. D. Li and S. Zhou, AIAA J. 34, 1097-1102 (1996)] or a streamlined body [S. P. Grace, H. M. Atassi, and W. K. Blake, AIAA J. 34, 2233-2240 (1996)]. The inversion techniques can also be applicable for the spatial transformation of sound field and active noise control. Finally, the possibility of the coupling of the inverse problem with computational aeroacoustics is to be discussed. [Work supported by NSF and ASF of China.] 


\title{
Session 2pNSe
}

\section{Noise: Computational Aeroacoustics III: Further Topics}

\author{
Tim Colonius, Cochair \\ Mail Code 101-44, California Institute of Technology, Pasadena, California 91125, USA \\ Eric Manoha, Cochair \\ Department of Computational Fluid Dynamics and Aeroacosutics, Office National d'Etudes et de Recherches Aerospatiales
} (ONERA), BP72, 92322 Chatillon, Cedex, France

\section{Contributed Papers}

\section{$5: 20$}

2pNSe1. Noise computation of a mixing layer using LES simulations. Christophe Bogey, Christophe Bailly, and Daniel Juvé (Lab. de Mécanique des Fluides et d'Acoust., Ecole Centrale de Lyon, France and UMR CNRS 5509 BP 163, 69131 Ecully Cedex, France)

A study of noise computation of a two-dimensional subsonic mixing layer is performed. The first concern is to develop a large eddy simulation (LES) code solving the filtered Navier-Stokes equations to obtain the nonlinear generation of sound. The compact DRP scheme of Tam and Webb is used in space, and the time integration is carried out by a fourstep Runge-Kutta algorithm. The boundary conditions of Dong and Tam are implemented. Then, a two-dimensional mixing layer is simulated. The spatial growth, turbulence rates, and spectra are in good agreement with other available studies. The goal is now to use the LES data to calculate the acoustic field generated by the mixing layer. Various methodologies have been investigated. First, the direct exploitation of the LES pressure field reveals tremendous difficulties due to the different orders of magnitude between aerodynamic and acoustic fluctuations. The other approaches consist of using source terms calculated from the LES velocity fluctuations. Two formulations are tested: Lighthill's analogy based on a volume integral over the region encompassing sound sources, and linearized Euler's equations where a source term is added. Numerical results and future developments will be discussed.

\section{5:40}

2pNSe2. An error analysis for the lattice gas time domain (LGTD) method in CAA. Victor W. Sparrow (Penn State Univ., Grad. Prog. in Acoust., 157 Hammond Bldg., University Park, PA 16802, sparrow@helmholtz.acs.psu.edu)

One of the most important requirements in computational aeroacoustics (CAA) is that a numerical method have excellent dispersion characteristics. The new lattice gas time domain (LGTD) method has better dispersion characteristics than any other scheme encountered by the au- thor. This talk will first review LGTD in comparison with the better known finite difference time domain (FDTD) method. Then quantitative error estimates will be provided for a benchmark problem from ICASE/ LaRC Workshop on Benchmark Problems in CAA [NASA Conf. Pub. 3300, 143-147 (1995)], showing the spectacular performance of the LGTD method. The present work answers some questions raised and extends the findings presented in CEAS/AIAA-95-115 [in Proc. 1st Joint CEAS/AIAA Aeroacoustics Conference, 12-15 June 1995, Munich, Germany, pp. 813-817] and in J. Comp. Acoust. 1, 423-454 (1994).

\section{6:00}

2pNSe3. Reduction of boundary effects in acoustic analogy application to CFD databases. Andreas Wilde and Marco Rose (Technische Univ. Dresden/Mommsenstr., 13/01062 Dresden, Germany, andreas@eakaw2.et.tu-dresden.de)

The calculation of noise generation and radiation using an acoustic analogy is very sensitive to the degree to which the sound-generating flow or rather the flow-simulation model meets the very strict assumptions made when deriving the aeroacoustic analogy. In many cases of technical interest, the popular assumption that there are no nonacoustic flow fluctuations on the boundary of the considered volume (control volume) is not satisfied. In these cases, the analogy has to be extended or an additional volume has to be attached, in which these nonacoustic fluid motions are damped. In this work, two examples of extension of the analogy formulation and of damping zones are studied. While all schemes yield reduction of noise prediction errors, the damping zone schemes are most effective in cancelling spurious noise sources. On the other hand, the extension of the acoustic analogies provides cost-effective ways to reduce noise prediction errors even at low frequencies. 
Session 2pNSf

\author{
Noise: Harmonization of Noise Ratings \\ Paul D. Schomer, Cochair \\ USA CERL, P.O. Box 9005, Champaign, Illinois 61826-9005, USA \\ Truls Gjestland, Cochair \\ SINTEF Telecom and Infomatics, O.S. Bragstads plass, Trondheim N-7034, Norway \\ Joos Vos, Cochair \\ TNO Human Factors Research Institute, P.O. Box 23, 3769ZG Soesterberg, The Netherlands \\ Chair's Introduction-2:15 \\ Invited Papers
}

2:20

2pNSf1. How many ways is it worth slicing the social survey data cake? Sanford Fidell (BBN Technologies, 21128 Vanowen St., Canoga Park, CA 91303)

As when serving portions of cake, social survey findings on the prevalence of noise-induced annoyance may be sliced in many ways to create dosage-response relationships. Schultz (1978) divided "clustering" from "nonclustering" surveys; FICON (1992) sliced off a few inconvenient data points; several researchers and agencies have found reasons to develop separate relationships for certain noise sources; while yet others question the rationale for developing dosage-response relationships in the first place. Are the various dosage-response relationships usefully different from one another, and artfully enough sliced to support regulatory policymaking or theory development? Might differences among dosage-response relationships inferred by different means reflect statistical artifacts, or the influences of nonacoustic factors? This paper discusses applied and other uses for different slices of the same cake, and how many (more) slices might be useful.

2:40

2pNSf2. Uncertainty in dosage-annoyance relations. Dick B. Botteldooren (Dept. of Information Technol., Univ. of Gent, St. Pietersnieuwstraat 41, 9000 Gent, Belgium) and Truls Gjestland (SINTEF Telecom and Informatics, N-7034 Trondheim, Norway)

Dosage-annoyance relations have traditionally been derived by fitting analytical curves through data gathered from large numbers of field studies. Information on the uncertainty of the relation thus derived is not often given. In this paper the same data set will be examined in several different ways and how this can influence conclusions will be demonstrated. The data set used is taken from Fidell and Barber [J. Acoust. Soc. Am. 89, 221-233 (1991)]. Transformations of annoyance scales and sound-level measures are assumed to be suitable and are not redone in this work. It is assumed that the average annoyance response of Man is searched and that the dosage-response curves will be used to integrate exposure levels to a single figure that can be used to compare noise impact to a community. This goal is totally different, with respect to uncertainty, from predicting noise annoyance in a particular exposure situation. In this work, individual data are combined, taking into account the different number of data points at different exposure levels and the uncertainty in results from individual studies. Each study is treated as an observation made by an independent expert. Differences to dosage-annoyance relations derived in a more traditional way are discussed. The obvious conclusion is that dosage response relations must be used with caution and that this can well be expressed by taking into account calculation uncertainty accurately.

3:00

2pNSf3. On using "loudness-weighted” SEL, LEQ, and DNL to assess noise environments. Paul Schomer (Schomer \& Assoc., 2117 Robert Dr., Champaign, IL 61821)

LEQ and DNL are commonly used to assess the long-term noise environments around airports, roads, etc. The fundamental building block to these assessments is the A-weighted sound level or SEL. These are used to compute LEQ or DNL. But many problems have surfaced over the simple use of A-weighting. The A-weighting has been shown to be deficient in assessing sound with strong low-frequency content, and other characteristics, like impulsiveness, are not properly accounted for by the A-weighting. However, no other simple filter has been shown to be superior to the A-weighting filter. One can consider building a more complicated filter that dynamically changes with level and frequency to better reflect human response than does the A-weighting. The equal loudness contours expressed in phons offers a set of curves that can be used to design such a filter. Like A-weighted sound level or A-weighted SEL, signals processed with such a new "filter" would be termed "loudness-weighted" sound level or "loudnessweighted" SEL. This author has analyzed many sounds to illustrate, test, and evaluate the concept of loudness-weighted SEL. This paper discusses the concept of using "loudness-weighting" in place of A-weighting and the results indicated by the analysis of many common sounds. 
2pNSf4. Soundscape in cities, limits to the noise limits? Tor Kihlman and Jean Berillon (Dept. of Appl. Acoust., Chalmers Univ. of Technol., SE-41296 Gothenburg, Sweden, tk@ta.chalmers.se)

Today traffic noise due to cars, trains, and airplanes is the main noise source in urban areas. In accordance to the European Commission's Green paper on Future Noise Policy, more than about 250 million people are exposed to A-weighted outdoor levels higher than $55 \mathrm{~dB}$. To improve the situation is a tremendous challenge and the question arises if we are really able to provide a good environment (i.e., levels below $55 \mathrm{~dB}$ ) for all people in today's cities. In a previous paper [Kihlman and Kropp, Proceedings International Congress on Acoustics, Seattle (1998)], different cities with different structures and automobile dependencies are compared in a general study based on statistical data. A flat regular town structure was assumed without shielding due to, for instance, buildings. In this paper the studies are extended to more common situations including detailed information about traffic flow and the screening of traffic noise due to the building structure. Results are compared with sound maps for different cities.

3:40

2pNSf5. Time-of-day penalty for aircraft noise. H. M. E. Miedema, R. G. de Jong, and H. Vos (TNO-PG, P.O. Box 2215, 2301 CE Leiden, The Netherlands, HME.Miedema@PG.TNO.NL)

Noise metrics differ in the penalty they apply to noise in the evening and the night, and in the definition of these periods. In $L_{\text {Aeq }}$ $(24 \mathrm{~h})$ no penalties are applied. DNL uses a penalty of $10 \mathrm{~dB}$ for the night $(22-7 \mathrm{~h})$, and DENL uses a penalty of $5 \mathrm{~dB}$ for the evening $(19-23 \mathrm{~h})$ and $10 \mathrm{~dB}$ for the night $(23-7 \mathrm{~h})$. An earlier meta-analysis [J. M. Fields, "The relative effect of noise at different times of the day-an analysis of existing survey data," Hampton, VA, NASA, Report CR-3965 (1986)] did not reveal consistent evidence on the basis of which penalties could be chosen. Data from the 1997 Amsterdan airport study [De Jong et al., "Hinder, Gezondheid- en Belevingsaspecten in de regio Schiphol," TNO-PG, Leiden, Netherlands, report 98.039 (1998); $N=11,812]$ are used to estimate the time-of-day penalties that give an optimal relation with noise annoyance and self-reported sleep disturbance, respectively. $L_{\text {Aeq }}$ 's are available for the following periods: $7-19,19-22,22-23,23-6$, and 6-7 h. Penalties are estimated for the whole sample, and for subgroups (e.g., age classes, different levels of noise sensitivity) separately.

4:00-4:20 Break

2pNSf6. Validation of a new procedure for rating shooting sounds with the help of field survey data. Joos Vos (TNO Human Factors Res. Inst., P.O. Box 23, 3769 ZG Soesterberg, The Netherlands, vos@tm.tno.nl)

In previous laboratory studies it was shown that an almost perfect prediction of the annoyance caused by shooting sounds was obtained on the basis of the outdoor A-weighted and C-weighted sound exposure levels (ASEL and CSEL; $L_{\mathrm{AE}}$ and $L_{\mathrm{CE}}$ ). For single events, the rating sound level, $L_{r}$, is given by $L_{r}=L_{\mathrm{AE}}+\beta\left(L_{\mathrm{CE}}-L_{\mathrm{AE}}\right)\left(L_{\mathrm{AE}}-\alpha\right)+12 \mathrm{~dB}$. The second term $\beta\left(L_{\mathrm{CE}}-L_{\mathrm{AE}}\right)\left(L_{\mathrm{AE}}\right.$ $-\alpha$ ) implies (1) that the annoyance is dependent also on the "heaviness" of the sound (characterized by $L_{\mathrm{CE}}-L_{\mathrm{AE}}$ ), and (2) that the additional annoyance $(\beta>0)$ increases with ASEL for $L_{\mathrm{AE}}>\alpha \mathrm{dB}$, and decreases with ASEL for $L_{\mathrm{AE}}<\alpha \mathrm{dB}$. In the present study, the procedure for rating shooting sounds, as described above, was validated with the help of data from a German field survey on the annoyance caused by artillery and road-traffic sounds: $\alpha$ and $\beta$ were determined for which $L_{r}$ for shooting sound would, overall, numerically correspond to the A-weighted day-night level of equally annoying road-traffic sound. For all relevant impulsive sources, the required ASELs and CSELs received in the various residential areas were determined by TNO Institute of Applied Physics. The results showed that a proper rating sound level was obtained with $\alpha=40 \mathrm{~dB}$ and $\beta=0.025$.

2pNSf7. A new test of the validity of a maximum single-event-level model in the prediction of the community response to artillery sounds. Edmund Buchta (Inst. für Lärmschutz, Arnheimerstraße 107, D-40489 Düsseldorf, Germany)

It is common, for many environmental sounds, to predict the community response from acoustic measures (such as $L_{\mathrm{eq}}$ or $L_{\mathrm{dn}}$ ) that take the yearly energy-average sound level into account. According to Rylander and colleagues, however, the annoyance is determined mainly by the level of the noisiest events [e.g., see J. Sound Vib. 127(3), 555-563 (1988); 192(1), 199-206 (1996)]. The number of those events is less important: the annoyance increases with the number of events up to a saturation point (e.g., for large firearms 1000 shots/year with $L_{\mathrm{CE}}>90 \mathrm{~dB}$ ) above which a further increase in number does not affect the annoyance. In the present paper, the usefulness of Rylander's model was tested with the help of data from a German field survey on the subjective effects of artillery sounds [J. Acoust. Soc. Am. (in press)]. The analyses showed that (1) application of a threshold to CSEL results in the loss of important information from residential areas without events above threshold, and (2) saturation, if any, might be observed only from about 70000 shots/year. It is concluded that for several reasons Rylander's model is inferior to models in which total exchangeability of single-event levels and number of events is assumed. 
Panelists: D. Botteldooren, Belgium

E. Buchta, Germany

S. Fidell, USA

T. Gjestland, Norway

T. Kihlman, Sweden

H. M. E. Miedema, The Netherlands

P. D. Schomer, USA

J. Vos, The Netherlands

TUESDAY AFTERNOON, 16 MARCH 1999

ROOM MA043, 2:00 TO 5:20 P.M.

\title{
Session 2pPAa
}

\section{Physical Acoustics: Thermoacoustics II}

\author{
Martin Wetzel, Cochair \\ BMW AG, Hufelandstrasse, 80788 Muenchen, Germany \\ Cila Herman, Cochair \\ Department of Mechanical Engineering, Johns Hopkins University, 3400 North Charles Street, Baltimore, \\ Maryland 21218, USA
}

\section{Invited Papers}

\section{2:00}

2pPAa1. Advances in pulse tube refrigeration. Ray Radebaugh (Physical and Chemical Properties Div., National Inst. of Sci. and Technol., 325 Broadway, Boulder, CO 80303)

The pulse tube refrigerator is now being developed for a wide variety of cryogenic cooling applications, and it is beginning to replace other types of cryocoolers in many of these applications. This widespread interest occurs because there are no moving parts in the cold end. Most of this development in pulse tube refrigerators began in the mid-1980s with the introduction of the orifice to provide the proper phase relationship between the gas velocity and the pressure. The pulse tube refrigerator has now become the most efficient cryocooler for temperatures of 60 to $120 \mathrm{~K}$. The current state-of-the-art in pulse tube refrigerators is discussed in this paper. Temperatures as low as $2.2 \mathrm{~K}$ with two stages and efficiencies as high as $19 \%$ of Camot at $80 \mathrm{~K}$ in one stage have been achieved. The operating fundamentals are explained very well through the use of thermoacoustic modeling techniques. Recent $R \& D$ areas to be discussed are the use of additional orifices, inertance tubes, tapered pulse tubes, dc flow, and thermoacoustic drivers. The fluid dynamics within the pulse tube is still not well understood, and further research in this area is needed to improve performance even further.

2pPAa2. Thermoacoustic instabilities of highly premixed flames in combustors and boilers. Thomas Sattelmayer and Christian Pankiewitz (Technische Univ. Muechen, Lehrstuhl A fuer Thermodynamik, Boltzmannstrasse 15, D-85748 Garching, Germany)

The attempt to reduce the formation of pollutants from combustion processes has led in recent years to the development of novel low-temperature combustion processes. Confined flames of this type exhibit a number of instability mechanisms, which can lead to unacceptable combustion noise or even to severe damage. The instability mechanisms, which appear in cases of technical interest, are reviewed and explained. It is shown that a realistic model for the description of the instability requires a proper representation of the contribution of the flame as the driving element. To date, nonlinear transfer functions have to be deduced from combustion experiments, but attempts to derive transfer functions numerically have already been published. In the past, modeling efforts were strongly focused on longitudinal modes. Although circumferential modes are of technical importance, they have not yet been fully understood. In this case, a complex interaction of the nonlinear flame with the linear acoustic system has to be taken into account and the transition between modes becomes more important. Since a satisfactory description of the interaction is not yet available, active instability control techniques have been proposed. Their limit in cases of technical interest will be discussed. 
2pPAa3. Thermoacoustic research at ETH Zürich in the 1990s. J. Bösel (Linde Kryotechnik AG, Dättlikonerstrasse 5, CH-8422 Pfungen, Switzerland) and Ch. Trepp (Swiss Federal Inst. of Technol. (ETH) Zürich, CH-8092 Zürich, Switzerland) $)^{\text {a) }}$

The linear thermoacoustic theory was developed by N. Rott, formerly Professor at the Institute of Aerodynamics at ETH Zürich, in the late 1960s. Since then a couple of research projects in that field have been carried out under the supervision of Prof. Rott, and more recently at the Institute of Process Engineering. The talk will give a short historical summary of the research activities at ETH Zürich and the latest work undertaken at the Institute of Process Engineering will be presented. This research was aimed at increasing the power density of thermoacoustic engines. Instead of the "stack," usually consisting of "long"” parallel plates, short parallel-plate segments were used, which were randomly oriented to each other. This alternative stack arrangement was expected to benefit from improved heat transfer characteristics and an anisotropic thermal conductivity. The results of a simple numerical model and experiments carried out with a thermoacoustic heat pump confirmed these expectations and will be presented in the talk. ${ }^{\text {a) }}$ Former affiliation.

\section{Contributed Papers}

\section{3:00}

2pPAa4. High-efficiency 2-kW thermoacoustic driver. Robert W. M. Smith, Robert M. Keolian, Steven Garrett (Grad. Prog. in Acoust. and Appl. Res. Lab., Penn State Univ., P.O. Box 30, State College, PA 16804), and John Corey (CFIC, Inc./Resonant Power Group, Troy, NY 12180)

A moving-magnet electrodynamic driver will be described which is intended to maintain an acoustic resonance within a thermoacoustic air conditioner containing an inert gas mixture of helium and argon at 30 bar. It is energized by a linear motor with a $\mathrm{B} l$-product of $41 \mathrm{~N} / \mathrm{A}$ and a coil resistance of $0.24 \Omega$. An auxiliary spring system augments the magnetic stiffness $(154 \mathrm{kN} / \mathrm{m})$ to provide a driver mechanical resonance frequency near $60 \mathrm{~Hz}$. An Inconel bellows forms a flexure seal which provides an effective piston area that maximizes electroacoustic conversion efficiency by making the Joule heating losses in the coil equal to the mechanical dissipation in the magnetic suspension. The driver's electroacoustic conversion efficiency is predicted to be in excess of $83 \%$. [Work supported by the Office of Naval Research.]

\section{3:20}

2pPAa5. Performance of a two-stack annular prime mover. Brent A. Carter and Anthony A. Atchley (Grad. Prog. in Acoust., Penn State Univ., P.O. Box 30, State College, PA 16804, aatchley@psu.edu)

In a previous study, numerical investigations of a two-stack annular thermoacoustic prime mover were made [H. T. Lin et al., J. Acoust. Soc. Am. 102, 3071(A) (1997)]. The conditions for onset were predicted. The subject of this paper is an experimental investigation of a two-stack annular prime mover. The prime mover, modeled using DeltaE and a MATLAB program, is constructed using an annular aluminum resonator, copper fin heat exchangers, and two parallel plate, glass stacks. The relative position of the two stacks is adjustable within the resonator. Fluid-cooled ambient heat exchangers, and cartridge-heater powered hot heat exchangers are used to apply independent temperature differences across the two stacks. The sound field and temperature within the resonator are measured with a series of microphones and thermocouples distributed along the resonator. The performance of the prime mover is investigated as a function of relative stack position, temperature differences applied to the stacks, and heater power levels. [Work supported by the Office of Naval Research and The Pennsylvania State University Applied Research Laboratory.]

\section{3:40-4:00 Break}

\section{4:00}

2pPAa6. On validity of mean-field approximation in thermoacoustics. V. Gusev (Laboratorie de Physique de l'Etat Condensé, UPRESACNRS 6087, Université du Maine, 72085 Le Mans, Cedex 9, France, vitali@laum.univ-lemans.fr), H. Bailliet, P. Lotton, and M. Brauneau (Université du Maine, 72085 Le Mans, Cedex 9, France)

Thermoacoustic heat flux is convective in its physical nature. In the present communication it is demonstrated that the problem of analytical description of thermoacoustic heat flux is identical to the problem of clos- ing moment hierarchy in the theory of fluid turbulence. In the case of harmonic acoustic oscillations, spatially inhomogeneous time-averaged temperature distribution is related to temperature oscillations at fundamental frequency. However, the first (fundamental) harmonic depends on the second harmonic of temperature field, which in turn is related to the third harmonic, and so on. In analytical models of thermoacoustic devices the harmonics of temperature oscillations are usually neglected. This so-called "mean-field" approximation results in the description of thermoacoustic heat transport as a diffusion process (with an effective thermal diffusivity depending on acoustic wave power). In the present communication it is demonstrated that mean-field approximation is not just inappropriate for the description of thermoacoustic heat exchange between gas-separated solid plates, but it is also not valid in all the cases where the characteristic spatial scale of mean temperature variation does not significantly exceed the amplitude of gas displacement in standing acoustic wave (for example, near the terminations of the stacks and heat exchangers). [Work is supported by DGA.]

\section{4:20}

2pPAa7. Second-order thermoacoustics. Roger Waxler (Grad. Prog. in Acoust., Penn State Univ., University Park, PA 16804, waxler@math.psu.edu)

A theoretical study is made of nonlinear effects in thermoacoustic stacks. The equations of fluid dynamics and heat transfer are expanded about a mean state with constant pressure and zero velocity but with a nonzero temperature gradient along the stack. The expansion is carried out to second order in Mach number. Using Rott's approach to control the linear terms, the time-independent parts of the second-order terms are studied. A set of coupled equations for the radiation pressure, velocity streaming, and time-averaged temperature are obtained and solved. The transverse heat flux into the stack is obtained. The solutions depend on the pressure and temperature at the ends of the stack, and thus on the details of the nonlinear effects in the resonator. However, it is found that the effects of the resonator on the velocity streaming and radiation pressure can be reduced to two parameters.

\section{$4: 40$}

2pPAa8. Nonlinear saturation of thermoacoustic instability. Sergey Karpov and Andrea Prosperetti (Dept. of Mech. Eng., Johns Hopkins Univ., Baltimore, MD 21218)

In earlier work [Watanabe et al., J. Acoust. Soc. Am. 102, 3484-3496 (1997)] a quasi-one-dimensional nonlinear model for thermoacoustic devices was developed. The model reduces exactly to the well-known Rott theory upon linearization, but numerical work has shown that it is also able to predict nonlinear features in agreement with experiment. In this work, a weakly nonlinear analysis of the model is carried out for conditions close to the linear stability threshold. The level at which the amplitude of the linearly unstable perturbation is predicted to saturate compares favorably with numerical calculations and experiment. On the basis of the result, the effect of several design variables such as nonuniformity of the resonator cross section, stack plate spacing, and others is explored. [Work supported by the Office of Naval Research.] 
gated through numerical calculations of the coupled acoustic, thermal, and shear fields near a corrugated interface, i.e., a surface with a profile $y$ $=A \cos q x$. The calculations were carried out in the limit where the acoustic wavelength is much larger than the scale of surface roughness $(A, 2 \pi / q)$ or the penetration lengths $\delta_{t}$ and $\delta_{v}$. Boundary layer phenomena cause phase shifts and energy absorption in the reflection of a plane acoustic wave by a smooth surface. These effects are further modified at a corrugated reflecting surface. The calculated reflection coefficients have been applied to the model relating the speed of sound to the frequencies of spherical and cylindrical acoustic cavity resonances, and to the theoretical model of the Greenspan acoustic viscometer. [Research supported in part by the Office of Naval Research.]

\section{Contributed Posters}

These posters will be on display in the Poster Gallery from Monday to Wednesday, 15-17 March. Authors will be at their posters from 10:00 a.m. to 12:00 noon on Wednesday, 17 March.

2pPAa10. How much pressure drop is necessary for heat transfer? I. Rülich and H. Quack (TU Dresden, Institut f. Energiemaschinen und Maschinenlabor, Lehrstuhl f. Kälte- und Kryotechnik, D-01062 Dresden, Germany, ingo@memkul.mw.tu-dresden.de)

In many technical applications, heat has to be transferred from a fluid to a wall. To do this efficiently in a short time and confined volume, the fluid has to be moved by applying a pressure difference. In refrigeration and power processes working with ideal gases, the pressure drop in the heat exchangers plays an important role in the overall efficiency of the cycle. The present work investigated whether the necessary pressure drop to obtain a certain amount of heat transfer can be derived from basic principles at least for Reynolds numbers below 2000. It was found that there exists a lower limit on the order of magnitude of 2 pressure heads per transfer unit. This figure is slightly dependent on the Prandtl number of the fluid. Real heat exchanger geometries have values higher than this minimum value, the magnitude depending on simple shape factors, which were derived from numerical fluid flow calculations and with published experimental data. This work identified how the ideal inner geometry of a regenerator for regenerative power and refrigeration processes should look like. [Work is supported by BMBF Grant No. 13N6619/0.]
2pPAa11. Threshold condition for traveling wave excitation in an annular prime-mover. T. Le Polles, S. Job, H. Bailliet, P. Lotton, M. Bruneau (Laboratoire d'Acoustique, IAM UMR 6613, Université du Maine, 72085 Le Mans, Cedex 9, France), and V. Gusev (Université du Maine, 72085 Le Mans, Cedex 9, France)

The threshold condition for thermoacoustic instability in an annular prime-mover is derived. The region of spatially inhomogeneous temperature distribution (due to the presence of a stack and hot and cold heat exchangers) is assumed to be acoustically thin. The interaction of acoustic waves with the stack is assumed to be quasiadiabatic. Theoretical approach (based on the transformation of differential wave equation into Volterra integral equation, which is solved by iterative procedure) is valid for arbitrary spatial temperature distribution. Nevertheless, it was found that the threshold for the excitation of a traveling acoustic wave does not depend on the details of temperature spatial distribution but just on the ratio of the temperatures of hot and cold heat exchangers. Moreover, the critical temperature ratio does not depend on the thickness of the stack if the acoustic losses inside the heated region are negligible in comparison with acoustic losses in the rest part of the annular system. In this important limiting case critical temperature ratio diminishes when the dimensions of the system are scaled up. [Work supported by DGA.] 


\title{
Session 2pPAb
}

\section{Physical Acoustics: Nonlinear Motion of Bubbles and Drops I}

\author{
R. Glynn Holt, Cochair \\ Department of Aerospace and Mechanical Engineering, Boston University, Boston, \\ Massachusetts 02215, USA \\ Joachim Holzfuss, Cochair \\ Institut für Angewandte Physik, TU Darmstadt, Schlossgartenstrasse 7, D-64289 Darmstadt, Germany \\ Chair's Introduction-4:15 \\ Invited Papers
}

4:20

2pPAb1. Acoustic levitation, a theoretical and experimental round up. E. G. Lierke (Battelle Ingenieurtechnik GmbH, Düsseldorfer Straße, 65760 Eschborn, Germany)

A resumé of terrestrial levitation of solid samples and drops in one-axial acoustic standing wave levitators is presented. It concentrates on a normalized theoretical description of drop displacement and deformation in the full dynamic range of the levitation safety factor, the ratio of axial levitation force and sample weight. The range of the initial sample radius $a_{0}$ follows from the wave number product $10^{-2}<k a_{0}<1.5$. Sample properties density $\rho$ and surface tension $\sigma$ are represented by the Bond number Bo= $=\rho / \sigma$ $\cdot a_{0}^{2} \cdot g_{0}<2$ ( $g_{0}$ is gravity constant). The critical Weber number $\mathrm{We}_{\text {crit }}=10$ (ratio of Bernoulli stress and capillary pressure) defines the upper limit of levitation at acoustic Mach numbers $\mathrm{Ma}_{\text {crit }}=\sqrt{5 / P_{0} \kappa_{0} \cdot \sigma / a_{0}}\left(P_{0}\right.$ is static pressure, $\kappa_{0}$ is heat capacity ratio). Resulting temperature variations and acoustic convections, represented by Nusselt and Sherwood numbers, are briefly discussed. A new family of modular, one-axial, acoustic levitators $(20,60$, and $100 \mathrm{kHz})$ with sensor-controlled, calibrated resonance tuning is introduced. The levitators can easily be changed from a "Bessel mode" tube version, with minimized gas volume, into an "open" version with free radial access to the sample, both optional with superimposed axial gas flow. Some experimental techniques with compatible commercial components, like temperature and humidity sensors, optical accessories (observation and spectroscopy), and microdrop injectors are briefly discussed.

4:40

2pPAb2. Physics of drop formation. Jens Eggers (Univ. of Essen, Dept. of Phys., 45117 Essen, Germany)

A wide variety of technologically relevant processes, like ink-jet printing, spray painting, or internal combustion, depends on a detailed understanding of the underlying mechanism of drop formation. The motion is driven by surface tension, which tries to minimize surface area, thus reducing the minimum radius of a fluid jet to zero in finite time. This singular motion is self-similar as a function of time, and independent of fluid parameters or of initial conditions. The theory is confirmed by experiment without adjustable parameters.

5:00

2pPAb3. Bubble dynamics and nucleation of ice in supercooled water. E. H. Trinh and K. Ohsaka (Jet Propulsion Lab., California Inst. of Technol., Pasadena, CA 91109)

The effects of mechanically induced impulses such as vibration and flow have been used to induce the solid-liquid phase transformation in liquids held below their equilibrium freezing point. Although processes used for grain refining in metallurgy rely on this artificially enhanced nucleation process, there is no clearly identified underlying physical mechanism. Experiments have been conducted with single bubbles immersed in a small volume of supercooled water and trapped in a $22-\mathrm{kHz}$ resonant cell to study the action of both large amplitude radial and shape oscillations. The experimental observations reveal that large-amplitude radial oscillations of micron-size air bubbles induce the nucleation of ice when a minimum supercooling of $5{ }^{\circ} \mathrm{C}$ is reached. Shape oscillations and radially nonresonant bubbles do not induce nucleation of ice within the same "incubation" time. The appearance of the ice crystal at the location of the trapped bubble strongly suggests a cause and effect correlation between the bubble radial oscillations and the freezing onset, and both sonoluminescing and nonluminescing bubbles appear to induce nucleation. This mechanism also has the additional benefit of allowing the direct measurement of free dendritic growth velocity within a supercooled liquid bulk in the absence of any artificially introduced seed. [Work sponsored by NASA.] 


\title{
Contributed Papers
}

2pPAb4. Acoustic diagnosis of singing cavitation vortices. Branko Bajic (Brodarski Inst., Holjevca 20, 10020 Zagreb, Croatia, bajic@t-online.de)

The acoustic field generated by hydrodynamic cavitation is usually random and broadband. There is, however, a case in which it is tonal. Indeed, as reported by Higuchi, Arndt, and Rogers [J. Fluids Eng. (Dec. 1989)], and Maines and Arndt [ASME Symp. Cavitation and Multiphase Flow (1995)], cavitating vortices which shed off from a body immersed in a flow may generate a harmonic acoustic signal even if involving no vibrations of the cavitating body. This paper presents the results of the parametric analysis of sound generated by the singing vortices produced in a test on a finite-span hydrodynamic profile immersed in a uniform water flow. A continuous development of the vortex through filling by air was investigated. The acoustic diagnosis of the process of oscillations of the cavitating vortex generating the singing is presented. The issues relevant to the theoretical modeling of the lock-in mechanism are investigated: necessary conditions for the onset and stability of the process, possible influence of water velocity other than through the cavitation number, influence of vortex energy, etc. The relevant peculiarities of the vortex dynamics are revealed. The question if there is a back-influence of the singing on the basic vortex dynamics is considered.

\section{$5: 40$}

2pPAb5. Radiation pressure of sound on moving vortices. Andrew G. Semenov, Vadim N. Alexeyev, and Samuel A. Rybak (N. N. Andreev Acoust. Inst., Russian Acad. of Sci., 4 Shvernik St., 117036 Moscow, Russia, bvp@acoins.msk.ru)

This report is devoted to the nonlinear acoustic pressure of sound waves propagating in the vicinity of a rather general class of localized flows induced by the motion of spherically symmetric structures of the type of vortices in the liquid, say, ocean environment. As a result, the mean force exerted on a vortex is considered. The conventional general expression for transport cross section derived in a nonlinear theory of interaction of sound with rigid particles is proven to be incorrect for the vortex-sound interaction case. For the specific case of Hill's vortex mean force, it is shown to be absent both in the traveling and standing sound wave fields. However, the force could be observable if the distribution of mean sound energy in the incident sound field in the limit of vortex dimensions could be characterized by nonzero spatial gradient. A generalized expression for mean force exerted on a localized vortex structure is derived.

\section{6:00}

2pPAb6. Bubble size distribution near a pressure antinode. Robert Mettin, Stefan Luther, Claus-Dieter Ohl, and Werner Lauterborn (Drittes Physikal. Inst., Univ. Göttingen, Bürgerstrasse 42-44, 37073 Göttingen, Germany, R.Mettin@physik3.gwdg)

When strong ultrasonic standing wave fields are generated in liquidfilled resonators, cavitation bubbles emerge and build dynamic structures, typically near the pressure antinodes. For technical applications like sonochemical reactions taking place in the resonator, the size distribution of the clustering bubbles is an important parameter. New photographic measurements of bubble sizes are presented, along with numerical size estimates. These are based on Bjerknes force and shape stability calculations for spherical bubbles. [Work partially supported by "Graduiertenkolleg Strömungsinstabilität und Turbulenz.'’]

TUESDAY AFTERNOON, 16 MARCH 1999

ROOM MA041, 2:00 TO 5:20 P.M.

Session 2pPAc

\section{Physical Acoustics: Interaction of Acoustic Fields with Suspended Particles II}

\author{
Ewald Benes, Cochair \\ Institut für Allgemeine Physik, Vienna University of Technology, A-1040 Vienna, Austria \\ Robert E. Apfel, Cochair \\ Faculty of Engineering, Yale University, 9 Hillhouse Avenue, New Haven, Connecticut 06520-8286, USA
}

Contributed Papers

2:00

2pPAc1. The acoustic generation of internal flow and rotation of levitated fluid particles. Eugene H. Trinh and Sang K. Chung (JPL/ Caltech, 4800 Oak Grove Dr., Pasadena, CA 91109)

The detailed motions of levitated fluid particles in response to acoustic fields have become a topic of renewed interest because of the potential application of containerless processing techniques to a number of fundamental studies in heat or mass transfer and crystal growth. In these situations, acoustic fields are generally used to generate internal circulation to boost the transport rates, or to control the rotational motion of a levitated solution droplet. Two instances where such effects have been discovered and are being exploited will be discussed. The first case involves millimeter-size gas or vapor bubbles ultrasonically trapped in a resonant liquid cell and displaying vigorous internal circulation due to the excitation of high-frequency capillary waves at their interface. The second example deals with the drive of rotational motion of levitated droplets in ambient air and the effects of viscosity on this coupling. The results indicate that acoustic streaming flows are responsible for the generation of this controlled torque. [Work sponsored by NASA.] 
2pPAc2. Whole blood clarification in an ultrasonic standing wave. Caroline M. Cousins, Susan E. Higgins, Medha S. Limaye, Jeremy J. Hawkes, W. Terence Coakley (School of Biosciences, Cardiff Univ., Cardiff CF1 3TL, UK), Peter Holownia, and Christopher P. Price (St. Bartholomew's and Royal London School of Medicine and Dentistry, London E1 2AD, UK)

There has been interest for a number of years in the possibility of separating, for transfusion proposes, blood cells from the suspending plasma in ultrasonic standing waves. On a much smaller scale, there has also been interest in separating cells and plasma so that the latter can be analyzed in single or multiple sample systems. Cells in whole blood occupy about $45 \%$ of the suspension volume. It has been shown with a number of different cell types, e.g., yeast and bacteria, that concentrations of this order are not as efficiently harvested by ultrasound as lower concentrations. Removal of cells from $2-5 \mathrm{ml}$ whole blood volumes has been examined here in standing wave fields from plane or tubular transducers. The tubular transducers were driven at frequencies from 0.66 to $3.4 \mathrm{MHz}$. A range of frequencies, sonication pulsing regimes, and power levels have been established that result in cell separation efficiencies in excess of 99.5\% in 5 min under conditions where cavitation has been avoided. The technique is now being applied to the blood of increasing numbers of volunteer donors to establish confidence in the ability to clarify plasma without cell damage. [Work supported by BBSRC and EU.]

\section{2:40}

2pPAc3. Viability of plant cells in ultrasonic fields. Hannes Böhm, Kenneth C. Lowe, L. Gregory Briarty, J. B. Power, Michael R. Davey (Life Sci. Dept., Univ. of Nottingham, University Park, Nottingham NG7 2RD, UK), and Ewald Benes (Vienna Univ. of Technol., A-1040 Vienna, AT Austria)

The influence of ultrasonic waves on suspension plant cell cultures (e.g., petunia and carrot) has been investigated. The dependency of viability, cell density, miototic division, and regeneration, on the controlled acoustic parameters' frequency of sound, energy density, treatment time, and wave type, is presented. Special focus is on the investigation of the influence of propagating plane waves, because these allow a spatially almost invariable acoustic pressure amplitude and, as a consequence, the same acoustic parameter values for all cells in the treated suspension volume. [Work supported in part by the European Commission, Contract No. ERBFMRXCT970156.]

\section{3:00}

2pPAc4. Viability of yeast cells in propagating ultrasonic waves. Stefan Radel, Aiden J. McLoughlin (Dept. of Industrial Microbiology, Univ. College Dublin, Dublin 4, IE Ireland, radel@iap.tuwien.ac.at), and Ewald Benes (Vienna Univ. of Technol., A-1040 Vienna, AT Austria)

In some recent papers the upper limits for the acoustic pressure amplitudes, in standing wave fields, that exert no measurable influence on viability of various biological cells have been reported. However, the pressure amplitude in a standing wave is periodically varying in space in the direction of sound propagation and, because of radiation pressure, the cells are not homogeneously distributed within the sample volume, resulting in effects arising from the dynamic environments created. Thus standing waves are obviously not appropriate to study the direct influence of sound pressure amplitude on viability data. Therefore, both metabolic activity/ viability of yeast cells and their ability to replicate as a function of acoustic frequency and sound pressure amplitude have been investigated in propagating waves. The observed effects are compared to those in crossed beam fields and in standing plane wave fields. The results allow a better forecast and interpretation of viability threshold data relevant in predicting the effects of acoustic parameters in standing wave fields. [Work supported in part by the European Commission, Contract No. ERBFMRXCT970156.]
2pPAc5. Ultrasonic standing waves to enhance optical particle sizing equipment. Ian L. Holwill and Andy T. Martin (DERA Porton Down, Salisbury, Wiltshire SP4 0JQ, UK, iljholwill@clara.net)

Experiments have been performed using dynamic light scattering (DLS) apparatus to investigate the potential of the use of ultrasonic forces for in situ removal of unwanted particles from the measurement volume. Megahertz frequencies were used to move micron-sized particles from the laser beam focus and leave the submicron particles for measurement. Removal of polystyrene latex (PSL) particles down to a diameter of $2 \mu \mathrm{m}$ was demonstrated while leaving particles with a diameter below $100 \mathrm{~nm}$ unaffected to be accurately measured by the DLS technique. Further experiments have been performed on airborne particles in custom-built single-particle light scattering apparatus. The aim of this study was to direct particles toward a laser focus for sizing by forward light scatter measurements. Sub- $100 \mathrm{kHz}$ standing waves were used to direct micronsized particles to a laser beam focus for analysis. Large particles (of the order of $20 \mu \mathrm{m}$ ) were very successfully moved toward the laser focus, and adjustment of the power to the transducers allowed continuous, real-time control of the concentration of particles delivered to the measurement volume. A concentration range of approximately 100:1 was achieved. For smaller particles below $10 \mu \mathrm{m}$, the concentration range was limited to 2:1.

\section{3:40-4:00 Break}

4:00

2pPAc6. Ultrasonically enhanced sedimentation-Results of an empirical scale-up approach. Johannes Spengler and Martin Jekel (Dept. of Water Quality Control, Sekr. KF 4, Tech. Univ. of Berlin, Strasse des 17, Juni 135, D-10623 Berlin, Germany, wrh@itu201.ut.TU-Berlin.de)

Ultrasonic standing waves are suitable for both coagulation of dilute suspensions and thickening of already settled sludge. For each purpose the right choice of process parameters and reactor design is important. The used ultrasonic effects are acoustic radiation and acoustic as well as hydrodynamic particle interaction forces. Acoustic and convective streaming cause shear stress on particle clusters and can therefore disperse aggregates. To achieve good efficiency at high flow rates, these undesirable streaming effects must be controlled, especially for the realization of larger reactors. One suitable measure is to divide the sonicated volume into several smaller parts separated by thin polyethylene films. Basic investigations in various batch and continuous flow lab systems led to an empirically derived scale-up concept. A prototype 3-MHz transducer array consisting of 16 single piezos used for coagulation has been developed and manufactured in cooperation with ELAC Nautik (Kiel). Preliminary results of two new pilot plants (sonicated volume $10 \mathrm{~L}$ for coagulation, respectively, $70 \mathrm{~L}$ for thickening) show good performance of the reactors. Comparison with previous lab scale data prove the applicability of the scale-up method as it will be presented at the meeting.

\section{4:20}

2pPAc7. Ultrasonic splitting of oil-in-water emulsions. Ralf König, Ewald Benes, Martin Gröschl (Vienna Univ. of Technol., Inst. für Allgemeine Physik, Wiedner Hauptstr. 8-10/134, A-1040 Vienna, AT Austria), and Jens Hald (Tech. Univ. of Denmark, DK-2800 Lyngby, DK Denmark)

Standing resonant ultrasonic wave fields can be utilized for liquidliquid separation of the dispersed particles and the fluid caused by the acoustic radiation pressure and the induced particle agglomeration or coagulation/coalescence process. For the splitting of oil-in-water emulsions, the available piezoelectric composite transducer technology was improved and a dedicated resonator with crossed plane wave sonication geometry has been developed. The resonator chamber is entirely made of aluminium or tempax glass and the PZT piezoceramic transducer delivers an acoustic energy flow density of up to $24 \mathrm{~W} / \mathrm{cm}^{2}$ into the sonication volume. The chosen resonance frequency is kept stable by automatic frequency control utilizing the maximum true power criterion. Physically and 
chemically well-defined low and high density pure laboratory and also industrially used cooling-lubricating oil-in-water emulsion samples have been investigated. The quality of the ultrasonic-induced particle separation/coagulation process is characterized by physical-chemical analysis of the separated oil- and water phase and by determining the change of the particle size distribution of the initial emulsion due to the ultrasonic treatment. [Work supported by the European Commission, Contract Nos. ERBFMBICT960916 and ERBFMRXCT970156.]

\section{$4: 40$}

2pPAc8. Acoustic deinking of paper: Pulse effectiveness in particle detachment. Sameer I. Madanshetty and Jogesh B. Chandran (Mech. and Nuclear Eng., 322 Durland Hall, Kansas State Univ., Manhattan, KS 66506-5106)

Deinking remains an important step in environmentally conscious manufacturing of paper. Removing xerographic ink from a print sample using acoustic microcavitation provides an easy method to observe context for studying cavitation-induced erosion at surfaces. Experiments indicate that microcavitation evolves microimplosions which are effective in causing deinking preferentially at ink sites. Acoustic microcavitation is brought about by low megahertz acoustic fields giving rise to micron-size bubbles that live a few microseconds. In exposing a surface to continuous waves for a defined duration, one could obtain cavitation effects in an average, overall sense; the details of nucleation, evolution of inertial events, and the precise interplay of field parameters in effecting cavitation, however, get glossed over. Studying pulsed cavitation using tone bursts at low duty cycles, instead of $\mathrm{CW}$ insonification, reveals interesting details of the initiation and evolution of acoustic microcavitation. Specifically, it is found that short-pulse deinking is more effective than long-pulse deinking.
This fact seems to raise an anomalous question of how the sample knows it is being visited by a short pulse or a long pulse. Recent experiments operationally demonstrate why short-pulse deinking is more effective. [Work supported by NSF.]

\section{5:00}

2pPAc9. Particle filtration by a combination of ultrasound standing waves and laminar flow. Jeremy J. Hawkes, Caroline Cousins, W. Terence Coakley (School of Biosciences, Cardiff Univ., Cardiff CF1 3TL, UK), and Peter J. Keay (Univ. of Luton, Luton, UK)

Radiation forces in ultrasonic standing waves move suspended particles to half-wavelength separations, thus creating concentrated bands of particles separated by cleared medium. Continuous flow filters of one-half wavelength acoustic pathlength $(0.25 \mathrm{~mm}, 3-\mathrm{MHz}$ frequency) are described here in which particles concentrate on the central axis of the flow chamber. This concentration is maintained downstream in a novel laminar flow expansion section where the concentrated particles and clarified suspending phase are removed at separate outlets. The expanded downstream laminar section facilitates the engineering and positioning of efficient removal ports. Filter construction provides observation windows for monitoring of band integrity either parallel or at 90 degrees to the transducer face. The short acoustic pathlength in the present systems allows particle separations at frequencies significantly higher than previously employed in single half-wavelength systems, thus permitting exploitation of higher acoustic pressures without generating order-disrupting cavitation. Results for clarification of polystyrene latex particles, under flow conditions where particles typically spend $<1 \mathrm{~s}$ in the field, are in excess of $98 \%$ and $70 \%$ for 9- and 1- $\mu \mathrm{m}$-diam particles, respectively. [Work supported by BBSRC and EU.]

\title{
Session 2pPAd
}

\section{Physical Acoustics: Cavitation Physics and Sonoluminescence III}

\author{
Werner Lauterborn, Cochair \\ Drittes Physikalisches Institut, Universität Göttingen, Bürgerstrasse 42-44, 37073 Göttingen, Germany \\ Ronald A. Roy, Cochair \\ Department of Aerospace and Mechanical Engineering, Boston University, Boston, \\ Masschusetts 02215, USA
}

\section{Contributed Papers}

\section{2:00}

2pPAd1. Experimental investigation of dynamics of sonoluminescing bubbles. Yuichiro Kato, Shigeo Hayashi (Dept. of Appl. Phys. and Chem., Univ. of Electro-Commun., Chofu, Tokyo 182-8585, Japan), Kenji Tanaka, and Hiroshi Kobayashi (Waseda Univ., Shinjuku, Tokyo 169-8555, Japan)

Light scattering was carried out for a cavitation bubble trapped in a spherical flask. A He-Ne laser of $7 \mathrm{~mW}$ was used as the light source and the scattering angle was $90 \mathrm{deg}$. A function generator was used as the trigger source and a digital phospher oscilloscope for averaging the data. Changing the drive voltage resulted in differences in the signal waveform above and below the sonoluminescence threshold; a sonoluminescing bubble had the maximum radius greater than a dark bubble did, but showed smaller rebounds. This suggests that energy concentration is made possible by tight and symmetric collapse of the bubble. Sonoluminescence signals were also retrieved for a few hours with no laser light, and a discontinous drift was noted in the appearance time on the oscilloscope, suggesting that a bubble may have drifted in space discontinuously. [Work supported by Grant-in-Aid for Scientific Research, Ministry of Education, Science, and Culture, Japan.]

\section{2:20}

2pPAd2. On the light emission from a collapsing laser-induced cavitation bubble. Claus-Dieter Ohl and Werner Lauterborn (Drittes Physikalisches Inst., Univ. Göttingen, Bürgerstrasse 42-44, Göttingen, Germany)

The collapse of laser-induced cavitation bubbles is accompanied by the emission of light. A detailed experimental study of the properties of the luminescence is presented. The dependence of the luminescence duration on the size of the bubble and on its sphericity prior to collapse is investigated. In aqueous sodium solutions line emission is found. The shape of these lines varies with the sphericity of the collapse. Also, differences in the emission spectrum in the case of spherical and aspherical collapse have been found. 


\section{2:40}

2pPAd3. Temperature dependency of luminescence from laser-produced cavitation bubbles. Olgert Lindau and Werner Lauterborn (Drittes Physikalisches Inst., Univ. Göttingen, Bürgerstrasse 42-44, D-37073 Göttingen, Germany)

Single laser-produced bubbles emit short light pulses in the collapse phase. This phenomenon is called single-cavitation bubble luminescence (SCBL) in analogy to single-bubble sonoluminescence (SBSL) where the bubbles levitate in an acoustic field. Laser bubbles are produced using a $Q$-switched Nd:YAG laser. The laser beam is focused with an aberrationminimized lens system into a temperature controlled cuvette filled with bidistilled water. A photomultiplier collects the light emitted in the collapse phase of the bubble. Bubble size is monitored with a hydrophone. As laser-produced bubbles are bigger in size than their acoustically driven counterparts, they emit stronger light pulses. The light energy per pulse in dependence on bubble size is reported. SBSL shows a strong dependency on temperature and a similar behavior is expected for SCBL.

\section{3:00}

2pPAd4. Single-cavitation bubble luminescence after fs-laser-induced breakdown in water. Cordt Schenke, Thomas Kurz, and Werner Lauterborn (Drittes Physikalisches Inst., Univ. Göttingen, Bürgerstrasse 42-44, D-37073 Göttingen, Germany, cordt@physik3.gwdg.de)

The use of ultrashort, intense laser pulses to induce an optical breakdown has both advantages and disadvantages. One of the most important advantages is the possibility to create cavitation bubbles with a much smaller size than by use of nanosecond pulses. By this, the parameter range of single cavitation bubble luminescence (SCBL) studies can be extended. The dynamics and luminescence of the created bubbles were investigated. Due to self-focusing, the initially formed plasma has a cylindrical shape, which leads to elongated cavitation bubbles. The observed dynamics of the bubbles seems to indicate the formation of two jets penetrating the bubble while collapsing. For the first time, luminescence of bubbles created this way is observed. It seems to arise from the collapse of microbubbles which are created by self-focusing in the prefocal region.

\section{$3: 20$}

2pPAd5. Time series analysis of cavitation bubble fields. Stefan Luther, Ulrich Parlitz, and Werner Lauterborn (Drittes Physikalisches Inst., Universität Göttingen, Bürgerstrass e 42-44, D-37073 Göttingen, Germany, luther@physik3.gwdg.de)

Acoustical cavitation bubble fields exhibit spatio-temporal structure formation on multiple scales involved. The temporal scales are the fast, medium, and slow scales given by the period of the driving, the typical relaxation time of the individual bubble motion, and the drift of the filaments, respectively. The spatial scales are the microscopic, mesoscopic, and macroscopic scales given by the bubbles' mean radii, typical bubble distances, and the wavelength of macroscopic disturbances (e.g., sound waves). Prominent examples of the observed structures are, for example, period doubling on a fast temporal scale and a slowly drifting filamentlike bubble distribution on the macroscopic spatial scale. These structures are experimentally investigated using simultaneously measured sequences of images (CCD camera) and acoustic time series (hydrophones). Based on these data the experimentally observed patterns are characterized using image processing techniques (e.g., principal component analysis) and by means of methods of nonlinear time series analysis (e.g., test for stationarity, dimension estimates, surrogate data analysis). The investigations focus on the correlation between spatial and temporal structures on different scales and transitions between them. [Work supported by "Graduiertenkolleg Strömungsinstabilität und Turbulenz.'’]

\section{3:40}

2pPAd6. Acoustic bubble traps. Reinhard Geisler, Thomas Kurz, and Werner Lauterborn (Drittes Physikalisches Institut, Universität Göttingen, Bürgerstr. 42-44, D-37073 Göttingen, Germany)

A small, oscillating bubble in a liquid can be trapped in the antinode of an acoustic standing wave field. Bubble stability is required for the study of single-bubble sonoluminescence (SBSL). The properties of the acoustic resonator are essential for the stable trapping of sonoluminescing bubbles. Its acoustic modes were measured by means of holographic interferometry and hydrophones. Flasks of different geometries (spherical, cylindrical, rectangular, ovoid) were investigated as well as different acoustic modes. Higher harmonic modes were used to trap several single, stable sonoluminescencent bubbles within one acoustic resonator (few bubble sonoluminescence, FBSL). This opens up the possibility to study bubble-bubble interactions (secondary Bjerknes forces).

\section{4:00}

2pPAd7. Vapor cavitation zone formation in a water layer. Isaac Shreiber (Inst. for Industrial Math., Hanachtom 4/2484311 Beer-Sheva, Israel, isaak@indigo.cs.bgu.ac.il)

This paper deals with a model for the formation of a vapor bubble cavitation zone within a water layer due to a rarefied wave propagating in the water layer. The water in the layer is close to the saturation line (subheated). Models are presented for vapor cavitation zone development, pressure field formation, and the phenomenon of coalescence. The specific feature of the cavitation is a reflected rarefied wave; the growing and floating bubbles generate the swelling of the entire bulk of water; the coalescing and floating bubbles can uplift the water layer and splash it. The gas cavitation phenomenon and a de-aeration technology are also discussed and the Epshtein model for cavitation was developed. The Epshtein model assumes that the essence of water cavitation is the solved gas diffusion into the bubble germs providing for their growth. The peculiarity of the problem is the bubbles get into a periodic acoustic wave and they grow under the rectified diffusion process activity. These large bubbles can escape the cavitating layer. 


\title{
Session 2pPAe
}

\section{Physical Acoustics: Surface Roughness}

\author{
Keith Attenborough, Cochair \\ Faculty of Technology, The Open University, Milton Keynes MK7 6AA, UK \\ Ali R. Kolaini, Cochair \\ ERC-OEL, University of California at Santa Barbara, 6740 Cortona Drive, Goleta, California 93117, USA \\ Chair's Introduction-1:55 \\ Invited Papers
}

2:00

2pPAe1. Measurements and models of small-scale surface roughness effects at audio-frequencies in air. Keith Attenborough (Faculty of Technol., The Open Univ., Milton Keynes MK7 6AA, England)

Surface roughness has been found to have an important influence on outdoor ground effect. Indoor and outdoor measurements of roughness effects are reported together with developments of analytical boss theories and numerical predictions using boundary elements. Although the Twersky boss theory including incoherent scatter is found to lead to reasonable agreement with data, it fails to predict measured diffraction grating effects and, overall, the BEM results are in better agreement.

2pPAe2. Experimental and modeling results for near-grazing propagation over rough surfaces. James P. Chambers and James M. Sabatier (Natl. Ctr. for Physical Acoust., Univ. of Mississippi, University, MS 38677)

Recent experimental work on acoustic propagation over rough surfaces has shown intriguing propagation effects ranging from frequency shifts in the ground interference dip to surface wavelike phenomena. Attempts to model the observed data with an effective impedance formulation have had some success but have met with some problems for certain propagation geometries and surface configurations. Experimental data and modeling results will be presented and discussed for a variety of surfaces ranging in surface properties from impermeable to loosely packed soil and ranging in surface roughness scales from low sloped perturbations to steeply sloped wedges.

2:40

2pPAe3. The influence of millimeter-scale seafloor roughness on measured and predicted high-frequency acoustic backscattering strength. Kevin B. Briggs (Seafloor Sci. Branch, Naval Res. Lab., Stennis Space Center, MS 39529), Darrell R. Jackson (Univ. of Washington, Seattle, WA 98105), and Steve Stanic (Naval Res. Lab., Stennis Space Center, MS 39529)

Seafloor microtopography, as measured by underwater stereo photogrammetry, is systematically characterized concurrently with other sediment properties as model parameters to be used for predicting high-frequency acoustic backscattering from the seafloor as part of field experiments. Typically, seafloor roughness is digitized at $2-5 \mathrm{~mm}$ intervals from 35- or 70-mm film transparencies with a stereocomparator capable of 1-mm-scale lateral precision and sub-millimeter-scale vertical accuracy. Relative seafloor height profiles are used to estimate roughness power spectra, which are parameterized as slopes and intercepts of the line fit to the roughness variance regressed on the spatial frequency. According to the composite roughness model developed by APL-UW, the decay of the roughness spectra with increasing spatial frequency (slope) and the spectral strength (intercept) can be used to determine the contribution of the seafloor roughness to the overall backscattering strength. Data from field experiments are presented in order to demonstrate the relationship between seafloor roughness at sub-centimeter scales and high-frequency acoustic scattering. Experimental acoustic data and model predictions are presented from a coarse, shelly sea floor off Jacksonville, Florida, a rippled fine sand in the Quinault Range off Washington, and a very fine sand with subtle seafloor roughness off Tirrenia, Italy. [Work supported by ONR.]

3:00

2pPAe4. Acoustic backscatter from a sandy seafloor with anisotropic roughness. Richard J. Brothers, Gary J. Heald, and Patricia A. G. Thomson (DERA Bincleaves, Weymouth, Dorset, UK)

A high-frequency array was used to gather backscatter data from a sandy seabed with an anisotropic roughness spectrum. The source and receiver were fixed to a pan and tilt head so that the orientation to the seabed could be varied. A significant reduction in the backscatter was observed as the transducer was trained from the across ripple direction to the along ripple direction. The variation in backscatter with respect to the grazing angle is compared with the composite roughness model and modifications have been incorporated to take account of the bedform roughness and local grazing angle. The wideband transducer employed allowed a range of bandwidths (up to $60 \mathrm{kHz}$ ) to be used, including short pulse and chirp waveforms. Variation in the number of elements in the beamformed data showed the variation in backscatter due to change in beamwidth. Supporting measurements of the experiment site were made by SACLANTCEN, including stereo photography and grab samples of the sediment. 
2pPAe5. Acoustic remote sensing of seafloor roughness: Resolving ambiguous estimates of roughness spectrum parameters. Christian P. de Moustier and Daniel D. Sternlicht (Marine Physical Lab., Scripps Inst. of Oceanogr., 9500 Gilman Dr., La Jolla, CA 92093-0205)

To predict or model how sound waves interact with the seafloor some parametrization of the roughness of the sediment-water interface is required. Conversely, bottom backscattered echoes contain information about the roughness of the interface, relative to the acoustic wavelength, often mixed with contributions from inhomogeneities in the sediment volume. However, inversions of seafloor roughness parameters from high-frequency $(10-100 \mathrm{kHz})$ acoustic backscatter measurements often produce ambiguous results with numerous plausible parameter combinations fitting a given acoustic backscatter angular dependence curve or an echo envelope shape. Based on the data and on direct or inferred seafloor roughness measurements reported in the literature, and assuming that the relief spectrum of seafloor topography obeys a power law, it is shown that a level of inversion ambiguity can be lifted by setting the roughness spectral exponent to a value appropriate for the sediment type and by iterating on the spectral strength and the allowable amount of sediment volume backscatter. This is illustrated with examples drawn from comparisons between seafloor echo envelope models and measured acoustic backscatter data yielding interface roughness spectral parameters and rms bottom curvatures consistent with the lithology of the areas investigated. [Work supported by ONR N00014-94-1-0121.]

\section{3:40-4:00 Break}

4:00

2pPAe6. Stochastic models for scattering by seafloor roughness. Darrell R. Jackson (Appl. Phys. Lab., Univ. of Washington, Seattle, WA 98105)

Models for acoustic scattering by seafloor roughness can be divided into numerical models that treat individual realizations of the random seafloor and stochastic models that provide statistical moments representing the scattered field. Such stochastic models require statistical submodels for seafloor roughness and incorporate acoustic submodels that connect statistics of the scattered field with statistics of the random interface. Thus, one must consider the compatibility of the interface and acoustic submodels. Recent work by various investigators has resulted in a number of improvements over classical perturbation and Kirchhoff results. A variety of seafloor types (e.g., fluid, elastic, layered) can now be treated, and improved acoustic approximations permit application over a wider range of roughness parameters. Questions remain, however, as to the accuracy of the newer models. [Work supported by ONR.]

\section{4:20}

2pPAe7. Characterization of small-scale seafloor roughness using close-range digital photogrammetry. Anthony P. Lyons, Warren L. J. Fox, Eric Pouliquen, and Tuncay Akal (SACLANT Undersea Res. Ctr., APO AE 0613-5000, lyons@ saclantc.nato.int)

Surface roughness is a fundamental property affecting a variety of physical phenomena including sediment transport and the interaction of acoustic energy with the seafloor. Characterization of bottom roughness and its dynamics is therefore essential for understanding and quantifying the influence of the sediment microtopography. Extensive field measurements of bottom roughness have been taken recently with an end-to-end digital photogrammetry system providing quantitative, two-dimensional surface roughness measurements on spatial scales of approximately a millimeter to a meter. Results of these measurements have shown that sediment surfaces in shallow water are often anisotropic and/or exhibit non-Gaussian height distributions, both of which have the potential to strongly affect seafloor acoustic scatter. For these kinds of surfaces, simple roughness parameters such as rms height or the slope and offset of a power-law representation of the power spectra will not give a sufficiently complete description. Twodimensional statistical models are needed to capture the anisotropic nature of sediments with oriented features, while for seafloors with peaked forms, it is the phase information in the frequency domain that is required, as this controls the shape characteristics of a surface. Characterization of seafloor roughness based on these ideas will be presented using results from the digital photogrammetry system.

\section{4:40}

2pPAe8. Measurement of the effective surface impedance of rough surfaces in laboratory and in situ. Christian Nocke (Fraunhofer-Inst. Bauphysik Stuttgart (IBP), Stuttgart Nobelstrasse 12, D-70569 Stuttgart, Germany)

The normal specific acoustic impedance fully characterizes the acoustic behavior of a surface. In this contribution, laboratory and in situ results of a novel measurement procedure for this impedance are presented. The acoustic propagation above the surface is most sensitive to the acoustical properties of the surface at small angles of incidence. This effect is utilized to deduce the impedance from measurements of the interference between the direct and reflected sound of a point source above the surface under investigation. For homogeneous and plane surfaces, the absorption coefficient calculated from these impedance values compares well with the results from tube measurements. For rough and/or inhomogeneous surfaces, the measured impedance results must be interpreted as effective values. The reliability of the method has been proved in laboratory measurements for a frequency range between $80 \mathrm{and} 4000 \mathrm{~Hz}$. Furthermore, results of the new method from room acoustical applications are presented.

\section{5:00}

2pPAe9. The influence of small-scale seafloor surface morphology on acoustic backscattering. Eric Pouliquen, Benoit Zerr, and Anthony P. Lyons (NATO SACLANT Undersea Res. Ctr., Viale S. Bartolomeo, 40019138 La Spezia, Italy)

Sediment transport induced by current flows and surface gravity waves often causes the formation of nonisotropic features of the seafloor interface. Depending on environmental conditions (e.g., current, sediment type, etc.), these directional ripples may display non-Gaussian or sharply peaked forms. Contrary to RADAR sea surface scattering, these two aspects of the morphology of the seabed 
interface have not been deeply considered in the study of scattering of acoustic waves from the seabed. Their influence on backscattering is performed using two-dimensional and three-dimensional models including a finite-difference time-domain model (FDTD), a model based on the integral equation method, and a model based on the Kirchhoff approximation. All these models compute the time response as well as an averaged scattering strength from realistic randomly generated surfaces and profiles. The results of the simulations and acoustic data at high frequency show a sensitivity of the backscattered signals and of the backscattering strengths to both the anisotropy and the sharp nature of the seafloor interface edges.

TUESDAY AFTERNOON, 16 MARCH 1999

ROOM H1012, 1:55 TO 6:20 P.M.

\title{
Session 2pPPa
}

\section{Psychological and Physiological Acoustics and Noise: Application of Psychoacoustics in Rating Environmental Noise}

\author{
Hugo Fastl, Cochair \\ Institut of Man-Machine Communication, Technical University of Munich, Arcisstrassse 21, D-80333 Munich, Germany \\ Manfred T. Kalivoda, Cochair \\ Wiener Gasse 146/3, A-2380 Perchtoldsdorf, Austria
}

Sonoko Kuwano, Cochair

Department of Environmental Psychology, Osaka University, 1-2 Yamadaoka, Suita, Osaka, 565-0871 Japan

Chair's Introduction-1:55

Invited Papers

2:00

2pPPa1. A cross-cultural study of the factors of sound quality of environmental noise. Sonoko Kuwano (Dept. of Environ. Psych., Faculty of Human Sci., Osaka Univ., 1-2 Yamadaoka, Suita, Osaka, 565-0871 Japan, kuwano@env.eng.osaka-u.ac.jp), Seiichiro Namba (Takarazuka Univ. of Art and Design, Japan), Mary Florentine (Northeastern Univ., Boston, MA), Zheng Da Rui (Academia Sinica, PROC), Hugo Fastl (Tech. Univ. Munich, Munich, Germany), and August Schick (Oldenburg Univ., Oldenburg, Germany)

The sound quality of various environmental noises was judged using semantic differential in Japan, Germany, the U.S.A., and China. The sounds used were aircraft noise, train noise, road traffic noise, speech, music, and construction noise. As the result of factor analysis, three factors were extracted in Japan and Germany. They were interpreted as "powerful," "'pleasant," and "metallic" factors. In the U.S., and China, "powerful" and "metallic" factors were not differentiated. The impression of "powerful", factors were found to show high correlation with $L_{\text {Aeq }}$ and loudness level based on ISO 532B. There seemed some difference in connotative meanings of the terms "loud," "noisy," and "annoying", among the four countries. There was little difference in the impression of high-level aircraft noise, train noise, and road traffic noise among the four countries. They were perceived as negative impression such as "annoying," "ugly," "unpleasant," etc. The validity of other psychophysical measures was examined in relation to these results.

2:20

2pPPa2. Individual noise sensitivity and performance on psychoacoustic tasks. Wolfgang Ellermeier, Monika Eigenstetter, and Karin Zimmer (Inst. fuer Psychologie, Universität Regensburg, 93040 Regensburg, Germany, wolfgang.ellermeier@psychologie.uni-regensburg.de)

In environmental noise surveys, self-reported noise sensitivity turned out to be a major predictor of noise-annoyance reactions. Its relationship to basic measures of auditory functioning, however, has not been systematically explored yet. Therefore, in the present investigation, a sample of 61 unselected listeners was subjected to a battery of psychoacoustic laboratory procedures ranging from threshold determinations to loudness scaling tasks. No significant differences in absolute thresholds, intensity discrimination, simple auditory reaction time, or power-function exponents for loudness emerged, when the sample was split along the median into two groups of "low" versus "high" noise sensitivity on the basis of scores obtained from a psychometrically evaluated questionnaire [K. Zimmer and W. Ellermeier, Diagnostica 44, 11-20 (1998)]. Small but systematic differences were found in verbal loudness estimates, and in ratings of the unpleasantness of natural sounds, thus suggesting that self-reported noise sensitivity captures evaluative rather than sensory aspects of auditory processing. 
2pPPa3. Loudness and annoyance of steady-state and temporally varying noise. Rhona Hellman (Dept. of Psych., Northeastern Univ., 360 Huntington Ave., Boston, MA 02115, hellman@neu.edu) and Norman Broner (VIPAC Engineers and Scientists Ltd., Melbourne, Australia 3207)

Loudness and annoyance judgments were obtained by magnitude estimation for low-frequency noises. In one experiment, listeners judged the loudness and annoyance of 10-s noise bursts; in a second experiment, loudness and annoyance judgments of a continuous noise were made at regular temporal intervals over an exposure period of $1 \mathrm{~h}$. All listening was through loudspeakers in an ASHRAE test room. For noise stimuli containing dominant low-frequency tones, the annoyance-to-loudness ratios ranged from 2.0 to nearly 8.0 under steady-state conditions. This large ratio variation was found despite nearly equal A-weighted levels. Moreover, consistent with a recent study [Hellman et al., J. Acoust. Soc. Am. 101, 2176-2185 (1997)], loudness decreased with time, more at low than at moderate sensation levels (SL). Perceived annoyance exhibited a similar dependence on SL with time, suggesting that loudness and annoyance are related. However, for noise stimuli with spectral peaks at 31.5 and $25 \mathrm{~Hz}$, annoyance declined by a smaller percentage than loudness. This effect produced an increase in the annoyance-to-loudness ratio over the 1-h listening period. [Work supported in part by ASHRAE.]

3:00

2pPPa4. Response to air-cooling machinery noise: A preliminary study. Nancy Franchek, Patricia Davies (1077 Ray W. Herrick Labs., Purdue Univ., West Lafayette, IN 47907-1077), Richard Wood, and Phil Lavrich (United Technologies Carrier, Syracuse, NY 13221)

People living close to commercial properties are often exposed to air-cooling machinery noise. Of primary concern is the awareness of tones in the sounds and how that awareness contributes to the overall annoyance experienced by people exposed to the noise. For noise control, it is most straightforward to control the strength of tonal contributions from components such as fans and compressors. For the class of machinery considered in this study, fan fundamentals are usually around $60 \mathrm{~Hz}$ and compressor fundamental frequencies are typically between 500 and $700 \mathrm{~Hz}$. A preliminary study was carried out using 27 sounds that simulated a broad range of possible noises made by this type of air-cooling machinery. The sounds were presented to people who were asked to rate them in terms of tonality and annoyance. Tonality and annoyance ratings only increased when sounds contained at least one compressor tone above a certain threshold level. High correlations were found between objective metrics and the average of the subjects' responses. Recommendations for future research include investigations into the individual effects of changing spectral balance, overall loudness, and tonal contributions for these types of sounds, because changing tonal levels alone affects all of these sound characteristics simultaneously.

$3: 20$

2pPPa5. Binaural assessment of loudness of environmental noise. Anna Preis (I. I. S. Univ. of Tokyo, 7-22-1 Roppongi, Minato-ku, Tokyo, 106 Japan)

Loudness of time-varying environmental noises (two types of car noise and aircraft noise) presented under dichotic and diotic stimulation was investigated via headphones. The stimuli were combinations of signals with increasing and decreasing SPL. The objective was to discover if there is a difference between the noise assessment of these signals presented in diotic and dichotic conditions. The results are compared with previous research, where it was found that for combinations of tone/noise and noise/noise, there was no difference between binaural loudness perceived in diotic and dichotic listening conditions. In the case of stimuli that consist of two tones of different frequency, those presented diotically were perceived to be louder than those presented dichotically.

\section{3:40}

2pPPa6. Aspects of global assessment of loudness-equalized interior car sound in stationary driving conditions. Thomas Hempel (Inst. of Commun. Acoust., Ruhr Univ., D-44780 Bochum, Germany)

In listening tests, loudness still turns out to be the most relevant parameter for a subject's assessment regarding interior car sound. In order to investigate further parameters in listening tests, the influence of loudness differences between the sound samples has to be avoided. Minimizing errors, this can be done by equalizing the loudness of presentation of each sample to the median of all samples in the set. It can be found that originally soft cars now yield lower rankings of preference than before. A possible reason might be the loudness of presentation, which is higher than in the real car. A presentation of sound samples, which now are equalized to the loudness originally appearing in those soft cars, could possibly lead to different rankings. Furthermore, global assessments of loudness-equalized interior car sounds were obtained using the method of paired comparisons as well as the method of magnitude estimation.

\section{4:00-4:20 Break}

\section{4:20}

2pPPa7. Psychoacoustic evaluation of noise emissions. Hugo Fastl (Inst. of Man-Machine-Commun., Tech. Univ. München, Arcisstr. 21, D-80333 München, Germany)

While the psychoacoustic evaluation of noise emissions is already on its way from basic research laboratories in universities to application labs in industry, the psychoacoustic evaluation of noise emissions is not yet widely applied [http://www.mmk.e-technik.tumuenchen.de/admin/noise.html]. Therefore, psychoacoustic evaluations of noise emissions from road traffic noise, railway noise, aircraft noise, industrial noise, and leisure noise are discussed. As a rule, the overall loudness of a noise emission is larger than the 
average of the instantaneous loudnesses of the acoustic events constituting the noise emission. With respect to physical measurements, statistical treatment of loudness values measured according to DIN 45631 can predict the subjective evaluations of noise emissions for different types of noises. [Work supported by DFG.]

4:40

2pPPa8. Evaluating sequences of environmental noise using the method of absolute judgment in laboratory and outdoor situations. Some methodological considerations. Juergen Hellbrueck and Alfred Zeitler (Catholic Univ. of Eichstaett, Environ. and Health Psych., Ostenstraße 26, D-85072 Eichstaett, Germany, juergen.hellbrueck@ku-eichstaett.de)

It has been shown that the perceived average loudness of a noise sequence can be predicted from the Leq [S. Namba and $\mathrm{S}$. Kuwano, J. Acoust. Soc. Jpn. (E) 1, 99-106 (1980)] or the percentile loudness $\mathrm{N}_{4}$ [e.g., H. Fastl, Contributions to Psychological Acoustics. Results of the Fifth Oldenburg Symposium on Psychological Acoustics (1991), pp. 205-216]. While most of the experiments have been conducted in laboratory situations, there is lack of evidence that the results can be transferred to noise situations outside of the laboratory. Context factors and psychological frames of reference play an important role, when environmental noises are rated in real noise situations. Category scaling based on the method of absolute judgment is regarded to be a natural scaling procedure which is inherently associated with psychological theories of frames of reference. Current results of empirical studies on the evaluation of longer periods of traffic noise have proved the applicability of category scales. The method has been used in outdoor settings as well as in laboratory situations, and yielded reliable results in both cases. Therefore, it is proposed to use measurements which are familiar to the subjects, such as absolute judgments, thus having direct access to individuals' perceptions.

5:00

2pPPa9. Application of loudness quantities in a field study. Ulrich Widmann (Müller-BBM, Robert-Kochstr. 11, 82152 Planegg, Germany) and Peter Lercher (Inst. für Social Medicine, Innsbruck, Austria)

The evaluation of noise emissions in field studies is mainly based on the concept of energy-equivalent A-weighted sound level. This averaging method has the advantage of being physically well defined and easy to compute. However, several laboratory studies showed that this method is aurally not adequate and the specific acoustic characteristics of traffic sources cannot sufficiently be discriminated. In a pilot study a good correlation was found between annoyance reactions of residents near a highway after noise abatement measures and perceived loudness. Residents' reactions near the highway could be well predicted using a percentile value of $N_{5}$. To predict the reactions for subjects living at larger distances from the source the effect of masking by environmental background noise has to be considered additionally using the psychoacoustic concept of partially masked loudness. The pilot study was restricted to a small number of inhabitants in one village. In a new field study in Austria it was attempted to confirm the preliminary results of the pilot study within a larger population. The ratings of loudness quantities, i.e., different percentile values, and of physical quantities, i.e., energy-equivalent A-weighted level and level percentiles, are investigated.

\section{5:20}

2pPPa10. Psychoacoustics and noise abatement in Austria-Some practical experiences. Manfred T. Kalivoda (Wiener Gasse 146/3, A-2380 Perchtoldsdorf, Austria, psia-consutt@eunet.at)

In Austria there are two main fields of application for psychoacoustic methods. For noise from trade and industry the Gewerbeordnung (trade act) does not use any SPL limits. There is a more stringent regulation that says that an industrial plant has to be licensed only if neither a health risk nor intolerable nuisance or annoyance can be expected. The Umweltverträglichkeits-PrüfungsGesetz (environmental assessment act) from the year 1994 includes industry and all modes of transport uses the same wording as the Gewerbeordnung. Nevertheless, A-weighted equivalent levels are frequently used for noise assessment in these two fields. There are many situations where it makes no big difference if $L_{\mathrm{A}, \mathrm{eq}}$ or psychoacoustics is used. However, there is about $10 \%$ to $20 \%$ of noise problems for which SPL alone is not adequate so wrong conclusions are drawn on the noise impact. psi-A Consult is very experienced in applying psychoacoustics on real noise problems. Some examples, showing the discrepancies between conventional and psychoacoustic noise assessment, are presented here.

5:40

2pPPa11. Factors influencing equal-loudness-level contours. Carsten Reckhardt, Volker Mellert (Physik Akustik Univ. Oldenburg, D-26111, Oldenburg, Germany), and Birger Kollmeier (Univ. Oldenburg, Oldenburg, Germany)

Data on equal-loudness-level contours taken from the literature show considerable differences between the results of different laboratories, even when using the recommended alternative forced-choice (AFC) method. Some of these differences can be attributed to free parameters in the procedures that the experimentator could change. This work investigates the influence of the experimental setup on the resulting curves obtained with an adaptive two-alternative forced-choice procedure for the 30- and 50-phon curves in the range from $200 \mathrm{~Hz}$ to $1 \mathrm{kHz}$. For the adaptive procedure, the amount of change in the resulting equal-loudness-level contours is measured for different free parameters (e.g., initial step size, starting level). It is shown that for a simple adaptive procedure the influence of the starting level is smaller than the range effect when using the method of constant stimuli. The effect of the starting level is reduced when using large initial step size in the adaptive procedure. However, the effect can still amount to $6 \mathrm{~dB}$. This effect diminishes when using an interleaved procedure. The maximum measurement error for the different procedures is estimated. In addition, it is shown that interindividual variance is reduced when the individual threshold is taken into account. 
2pPPa12. Effects of helicopter and aircraft interior noise and vibration on passengers' comfort sensation and subjective well-being. Julia Quehl, August Schick (Dept. of Psych., Inst. for Res. into Man-Environment-Relations, Univ. of Oldenburg, 26111 Oldenburg, Germany, quehl@uni-oldenburg.de), Volker Mellert, Brigitte Schulte-Fortkamp (Univ. of Oldenburg), and Hermann Remmers (Inst. for Tech. and Appl. Phys. (ITAP) GmbH, Oldenburg, Germany)

Apparently passengers' sensation of comfort and subjective well-being are key concepts in the research on user acceptance of helicopter and aircraft systems. The design and development of advanced air transportation technologies require a fundamental, theory-based understanding of these concepts. From the passengers point of view, helicopter and aircraft interior noise and vibration are primary physical factors underlying the perception of comfort and well-being. In various judgmental contexts the influence of exposure to combined interior noise and vibration in helicopters and aircrafts will be analyzed by means of combined psychoacoustical, socioacoustical, and psychological research methods: Field experiments in a flight simulator and during real flight situations as well as laboratory experiments using a sound and vibration reproduction system will be carried out in order to examine the interactive effects of noise and vibration. The aim is to develop a passenger comfort or well-being index for helicopter and aircraft interior environments. The index will be based on the integration of physical predictors for the flight situation and psychological predictors for the passengers' sensation of comfort and well-being.

TUESDAY AFTERNOON, 16 MARCH 1999

ROOM H106, 2:00 TO 6:20 P.M.

\title{
Session 2pPPb
}

\section{Psychological and Physiological Acoustics: Physiological Acoustics}

\author{
Glennis R. Long, Chair \\ Department of Audiology and Speech Sciences, Purdue University, West Lafayette, Indiana 47907, USA
}

\section{Contributed Papers}

2:00

2pPPb1. A new HRTF decomposition and the corresponding algorithm for sound spatialization. Eric Dudouet and Jacques Martin (Ctr. Scientifique et Technique du Batiment, 25 rue J. Fourier, 38400 Saint-Martin-d'Heres, France)

Binaural simulations make extensive use of head-related transfer functions (HRTF), so spatializers have to deal with large amounts of data and long computing times to interpolate the HRTF database over the whole space. A better understanding of human auditory localization could lead to a better simulation of localization cues and allow the database and the computing time to be minimized. Principal component analysis on HRTFs, reported by D. J. Kistler and F. L. Wightman [J. Acoust. Soc. Am. 91, 1637-1647 (1992)], gave only second-order decorrelated base vectors. In order to find simple vectors (bandpass or low-pass), an independent component analysis has been computed for upper-hemisphere preprocessed HRTFs (reported in by M. Emerit et al. [Proc. 15th ICA, Trondheim, Norway, (1995) pp. 437-440]). New analyses have been done on a new HRTF data set, on different representations (time, magnitude alone and with phase). These analyses give a small number of base vectors, which can be used to reconstruct any HRTF, with a correlation coefficient near 1 , and allow a new spatialization method using 3 IIR filters, derived from independent impulse responses, to be conceived. Source signals are filtered by each of these filters, and linear combination of the results gives the spatialized signal.

\section{2:20}

2pPPb2. Vibration characteristics of bone-conducted sound. Stefan Stenfelt, Bo Hảkansson (Dept. of Signals and Systems, Chalmers Univ. of Technol., Göteborg, Sweden, stenfelt@s2.chalmers.se), and Anders Tjellström (Sahlgrenska Univ. Hospital, Göteborg, Sweden)

A dry skull, prepared with a layer of damping material in the brain capsule, was used to investigate the vibratory pattern of bone-conducted sound. Three orthogonal vibration responses of the cochleas were measured, by means of accelerometers, in the frequency range 0.1 to $10 \mathrm{kHz}$. The exciter was attached at different points in the temporal, parietal, and frontal bones. The results revealed a profound low-frequency antireso- nance (attenuation) in the ipsilateral transmission path, which was psychoacoustically shown to yield a distinct lateralization effect. It was further shown that the direction of excitation coincides with that of maximum response at the ipsilateral cochlea. No dominating response direction was found for frequencies above the first skull resonance at the contralateral cochlea. An overall higher response level was achieved at the ipsilateral cochlea when the transducer was attached close to the posterior semicircular canal compared with the other excitation points of the skull, for the energy transmission in general and specifically for the direction of excitation. Transcranial attenuation between the cochleas was found to be highly frequency specific, with a maximum of $40 \mathrm{~dB}$ in a single direction. Transcranial energy attenuation was less than $10 \mathrm{~dB}$ for the investigated frequency range.

\section{2:40}

2pPPb3. Estimation of the sound pressure at the eardrum. Herbert Hudde (Inst. of Commun. Acoust., Ruhr Univ., Bochum, Germany) and Andreas Engel (St. Elisabeth Hospital, Bochum, Germany)

In hearing experiments or in audiological investigations, the results usually have to be referred to an appropriate input signal. Typically, the input voltage of a headphone or the sound pressure generated in a free sound field or under a different reference condition are used. In many cases, however, it would be preferable to know the sound pressure at the eardrum, as it is more closely related to the auditory sensation. If headphones are used, the reference to the pressure at drum can be approximately achieved by means of an ear simulator, such as the well-known ear simulator, according to DIN IEC 711. But the ear simulator can only reproduce the acoustical properties of an average ear. The resulting errors depend on various parameters, including those of the headphone used. A considerable portion of the error is caused by the individual ear canals. These errors alone can exceed $10 \mathrm{~dB}$ in the audiologically used frequency range up to $8 \mathrm{kHz}$. Some methods of estimating the pressure at the drum are investigated. Optimum results are obtained if both the sound pressure and the volume velocity at the ear canal entrance are measured. But fairly good results can also be achieved without measuring the volume velocity. 
ture, and human subjects who have fine structure. Similarities and differences between the species are used to evaluate this model of cochlear fine

2pPPb4. Three-dimensional measurements of a physical full-size model of the human middle ear. Henning Taschke (Inst. of Commun. Acoust., Ruhr Univ., Bochum, Germany) and Christian Weistenhofer (Ruhr Univ., Bochum, Germany)

Complete systematical investigations of the vibrational properties of the human middle ear are impossible to carry out by means of temporal bone measurements of a single specimen because of post-mortem changes (time variance), damages to the object to be measured, and the irreversibility of manipulations. Measurements of physical models offer the facility for examining the properties of the human middle ear regardless of the above problems. The quality of the scientific findings obtained from the physical models depends on how good the properties of the real middle ear are reproduced by the elements, forms and materials chosen. A physical full-size model of the human middle ear was developed that allows the use of measurement instrumentation which is applied for real ear measurements. One- and three-dimensional measurements of the physical full-size model will be presented. The quality of the model will be judged by comparisons with real ear measurements and model predictions. The fields of application of the model resulting from this will be discussed.

\section{3:20}

2pPPb5. A general model of inner ear pressure responses including ultra-low frequencies. Ernst-J. Haberland (Dept. of ENT and HNS, Univ. of Halle-Wittenberg, Magdeburger Str. 12, D-06097 Halle, Germany) and Hans J. Neumann (City Hospital Martha-Maria, D-06120 Halle, Germany)

Various volume displacement processes act on the inner ear fluids, e.g., via the stapes, via the capillary bed, via the cochlear aqueduct or in the case of ultra-slow processes via pressure homeostasis from tissue. Frequencies below the audible range make a pressure difference in the perilymphatic scales but without any audible traveling wave dispersion. In experiments in guinea pigs, interference patterns were found between audible and low-frequency nonaudible perilymphatic flows in CM recordings. The function of perilymph can be subdivided into the dispersional part and the conventional part. Franke and Dancer [Arch. Otorhinolaryngol. 234, 213-218 (1982)] described a model of the guinea pig's cochlea in accordance with pressure measurements in the two perilymphatic scales up to low frequencies at the lower boundary of the audible range. In our own experiments, it was measured pressure responses to slow and ultraslow changes of perilymphatic infusions and aspirations and superimposed incremental volume pulses into the perilymphatic system [Neumann and Haberland, Biophysikalische Untersuchungen (Tectum Verlag, Marburg, 1998)]. From these experimental findings was developed an extended model of the passive pressure characteristics of the inner ear system that takes into account nonlinearities and valve characteristics in the convection-relevant slow and ultra-slow movements of perilymph.

\section{3:40-4:00 Break}

\section{4:00}

2pPPb6. Cochlear fine structure in chinchillas. Glenis R. Long, Lauren Shaffer (Dept. of Audiol. and Speech Sci., Purdue Univ., West Lafayette, IN 47907), William J. Murphy (Natl. Inst. for Occupational Safety and Health, Cincinnati, OH 45226), and Carrick L. Talmadge (Univ. of Mississippi, University, MS 38677)

Spontaneous otoacoustic emissions (SOAEs) and distortion product otoacoustic emissions (DPOAEs) were evaluated in both ears of nine apparently normal-hearing chinchillas selected because preliminary screening indicated that they had SOAEs in at least one ear. All chinchillas show DPOAE fine structure in both ears (even in ears without detectable SOAEs). The spacing of the SOAEs and the characteristics of DPOAE fine structure are compared with OAE measures (obtained using the same procedures) from two species of kangaroo rat (Dipodomys merriami and Dipodomys spectabilis) that have no SOAEs and no DPOAE fine struc- structures further [Talmadge et al., J. Acoust. Soc. Am. 98, 1517-1543 (1998)].

\section{$4: 20$}

2pPPb7. Modulation of distortion product otoacoustic emissions (DPOAE) by low-frequency masker tones in human ears. Torsten Marquardt (Inst. of Sound and Vib. Res., Univ. of Southampton, Southhampton, England), Johannes Hensel, Günther Scholz, and Dieter Mrowinski (Humboldt Univ., Berlin, Germany)

Biasing of the basilar membrane displacement by a loud lowfrequency tone causes changes of the DPOAEs depending on the phase of the masker. A corresponding model helps to understand the nonlinear behavior of the cochlea and to improve the diagnosis of inner ear diseases. Up to now, short time Fourier transform has been used to measure the DPOAE suppression pattern. This method has inherent time and frequency resolution limits. Therefore a new approach is presented, where the primaries and all frequencies generated by the process are harmonics of the masker. A rectangular time window with length according to the masker period is used. The part of the spectrum representing the DPOAE signal is regarded as modulated tone, consisting of a DPOAE frequency $(2 f 1-f 2)$ and spectral side frequencies. The time course of the DPOAE suppression pattern is computed by superposition of these complex components. The results are compared with a simulation of the distortions of the outer hair cells' mechano-electrical transfer function. In addition, a cochlear impedance model is verified. Variation of masker frequency results in the expected phase shift of the DPOAE modulation pattern and allows the assessment of the lower cutoff frequency of the human cochlear impedance.

\section{$4: 40$}

2pPPb8. Characteristics of amplitude modulation following responses in man. Günter Mauer and Wolfgang H. Doering (Univ. of Technol., RWTH Aachen, ENT-Dept., Audiol., Pauwelsstr. 30, D-52057 Aachen, Germany, mauer@alpha.imib.rwth-aachen.de)

Amplitude modulation following responses (AMFRs) are a subset of envelope following responses (EFRs) and can be recorded from the human scalp. In AMFRs continuous amplitude-modulated (AM) stimuli with carrier frequency $f_{c}$ and modulation frequency $f_{m}$ are used. Responses arise at frequency $f_{m}$ and are largest for modulation frequencies in the range below $100 \mathrm{~Hz}$ depending on the subject's age and state of arousal. Multichannel recordings and brain electrical source analysis were carried out to get information about localization of AMFR generators and dependency on modulation frequency of the stimulus. Recording of AMFR seems to yield important information about frequency-specific hearing loss even in the low-frequency range. Responses are assumed to be evoked by neural excitation originating mainly from a basilar membrane region corresponding to the carrier frequency $f_{c}$. However, in masking experiments, it could be shown that also regions of the basilar membrane related to frequencies up to several octaves above the carrier frequency contribute to the responses although stimulus bandwidth is very narrow. Models of cochlear mechanics were used to elucidate stimulus-response characteristics and effective stimulation bandwidth. Thresholds of AMFR in normal-hearing and hearing-impaired subjects are in close relation to psychoacoustically determined thresholds for the same stimuli.

\section{5:00}

2pPPb9. Excitation and inhibition in the dorsal nucleus of the lateral lemniscus influence binaural response properties of neurons in the rat's inferior colliculus. Jack B. Kelly (Lab. of Sensory Neurosci., 329 Life Sci. Bldg., Carleton Univ., Ottawa K1S 5B6, Canada, jkelly@ccs.carleton.ca)

In vitro brain slice studies have shown that neurons in the rat's dorsal nucleus of the lateral lemniscus (DNLL) are affected by both NMDA and non-NMDA receptor-mediated excitation and glycineric inhibition evoked by electrical stimulation of ascending afferent fibers in the lateral lemnis- 
cus and GABAergic inhibition evoked by stimulation of the commissure of Probst [Wu and Kelly, 1996]. The effects of excitatory and inhibitory events in the DNLL on binaural responses in the rat's inferior colliculus are reported here for in vivo experiments. Receptor specific antagonists were injected locally into the DNLL before and after recording extracellular single-neuron responses from the contralateral inferior colliculus. Binaural responses were evoked by paired clicks delivered to the two ears. Both NMDA and non-NMDA excitatory amino acid antatonists (NBQX and $\mathrm{CPP}$ ) produced a release from binaural inhibition and glycinergic and GABAergic antagonists (strychnine and bicuculline) resulted in enhanced binaural inhibition. The results support the idea that the DNLL plays a role in binaural processing through an inhibitory (GABAergic) influence on the contralateral central nucleus of the inferior colliculus. [Research supported by NSERC of Canada.]
2pPPb10. Sensitivity of auditory units in the frog's midbrain to amplitude decrements. Nikolay G. Bibikov (N. N. Andreyev Acoust. Inst., Shvernik st. 4, Moscow 117036, Russia, BVP@ASU.ACOINS.MSK.SU)

Responses to single and repetitive amplitude decrements in an otherwise steady best-frequency sinusoid were recorded in midbrain auditory units of the curarized grass frog. The modulators were either 1 or 12 full cycles of cosine function (frequencies: $10-100 \mathrm{~Hz}$, maximal modulation depths: $6 \%-80 \%$ ). All units were also classified according to their responses to pure tone and AM tone bursts. In some tonic units, which demonstrated enhancement of synchronization in the course of AM tone bursts, enhancement of the rate response to successive amplitude decrements was routinely observed. The response to the first decrement in succession could be omitted, but the probability of highly synchronized firing increased gradually to the end of the series of decrements. The effect could be explained by adaptation of the unit to a new average intensity level. Some onset units, which did not respond to any modulation periods of $100 \%$ AM tone bursts, demonstrated a prominent response to a single $10 \%-20 \%$ decrement in a pure tone. The dependence of the response upon duration of decrement (period of a cosine function) usually showed a prominent maximum in the range of $20-50 \mathrm{~ms}$. The response latency increased with the decrement duration, showing that the firing was evoked by an amplitude recovery.
2pPPb11. Incremental responses in the auditory cortex and the auditory periphery. Robert L. Smith, Dyanne Baptiste (ISR and Dept. of Bioeng. and Neurosci., Syracuse Univ., Syracuse, NY 13244-5290, Bob_Smith@isr.syr.edu), and Bernd Lutkenhoner (Univ. of Munster, Munster, Germany)

When probe tones are added to background sounds, the perception or detectability of the probe is influenced by the time delay from the onset of the background to the onset of the probe. In general, the shorter the time delay, the harder it is to detect or perceive the probe as a separate signal. Possible physiological counterparts of these phenomena were investigated by adding 40 -ms-long probe tones to $200-\mathrm{ms}-1$ ong background tones. The corresponding increment in response was measured in the auditory nerve of anesthetized gerbils, using single-unit and modulation following responses, and in the auditory cortex of awake humans, using a 37-channel Magnetoencephalogram (MEG) and a single equivalent current-dipole model. The auditory nerve responses appear to obey superposition such that the increment in response is independent of the time delay. In contrast, the increment in the MEG response was significantly smaller for small delays than for long delays. The results suggest that the cortical responses behave differently than the peripheral responses, and more closely resemble those obtained psychophysically, although differences in experimental paradigms may also affect comparisons. Hence the difference between peripheral and central effects may reflect a functionally significant change in temporal processing occurring along the auditory pathway.

\section{6:00}

2pPPb12. Analyses of continuous brain waves in relation to subjective preference of the sound field. Chiung Yao Chen (Dept. of Architecture, Chaoyang Univ. of Technol., 168 Gifeng E. Rd., Wufeng Taichung, Taiwan, ROC)

Two factors comprising a standard of time are prevailing for designing a music hall: (1) initial time delay gap between the direct sound and the first reflection $\left(\Delta t_{1}\right) ;(2)$ subsequent reverberation time $\left(T_{\text {sub }}\right)$. In brain researches of sound cognition, auditory-evoked potential (AEP) was frequently applied. However, it is undetectable in studying the variation of $T_{\text {sub }}$ and $\Delta t_{1}$ by a longer stimulus. In the present study, a method for measuring a human's responses on continuous brain waves (CBW) was arranged with respect to the subjective preference of a music sound field. First, the "effective duration" $\left(\tau_{e}\right)$ was defined by the effective gap of the initial deduction (0.1 envelope) of the autocorrelation function of CBW. Results show the values of $\tau_{e}$ efficiently prolong $\left(p_{i}=0.05\right)$ on the left hemisphere when the scale values (SVs) of preference increases by changing $\Delta t_{1}$. Second, this relationship was extended to examine changing $T_{\text {sub }}$. The longer $\left(p_{i}=0.01\right) \tau_{e}$ were found as the SVs increased in the range of $T_{\text {sub }}=0.2$ to $1.2 \mathrm{~s}$. Finally, as the auditory tempo was varied, the differences of values of $\tau_{e}$ show a closer correlation $\left(r=0.80, p_{i}\right.$ $=0.01$ ) to the differences of SVs on the left hemisphere. 


\title{
Session 2pSAa
}

\section{Structural Acoustics and Vibration: Structural Vibration, Radiation and Scattering I}

\author{
Mauro Pierucci, Cochair \\ Department of Aerospace and Engineering Mechanics, San Diego State University, San Diego, California 92182-1308, USA \\ Christiaan Kauffmann, Cochair \\ Faculty of Technology, Mathematics, and Informatics, Delft University of Technology, P.O. Box 5031, 2600 GA Delft, \\ The Netherlands
}

Contributed Papers

2:00

2pSAa1. On the acoustic Zeeman effect. Christiaan Kauffmann (Faculty of Tech. Math. and Informatics, Delft Univ. of Technol., P.O. Box 5031, 2600 GA Delft, The Netherlands, kauffman@dv.twi.tudelft.nl)

Thin, square plates subject to uniform boundary conditions vibrating in flexural motion have an enumerable set of resonances, some of them having multiple resonant modes. This holds for plates vibrating in vacuo. If the plate motion is subject to fluid loading, the resonant properties modify. Fluid loading induces all resonances to shift downwards (added mass effect), while acoustic radiation contributes to the damping of the modes. These features are well known and understood, although the actual calculation requires considerable computational effort. A perturbation method is presented for dealing with the effect of light fluid loading on the degenerate resonances. Removal of degeneracy is found for repeated modes having different coefficients for added mass and radiation damping. Symmetry is preserved for degenerate resonances that are modified by fluid loading in an identical way. Numerical results are presented that show how resonances split for the modes $u_{m n} \pm u_{n m}$ of simply supported plates, where $m$ and $n$ have the same parity, i.e., $m+n$ is even. Simple physical arguments, like equivalent multipole sources of the mode shapes and their spatial modulation, are used to interpret the results. Mode shapes with the highest degree of symmetry show the largest shift in frequency.

\section{2:20}

2pSAa2. HYPERION-A computational tool for the investigation of the sound transmission loss of periodically structured plates. Waldemar Maysenhoelder, Bozica Horvatic, and Klaus Nasshan (Fraunhofer-Inst. f. Bauphysik, Nobelstr. 12, D-70569 Stuttgart, Germany, maysenhoelder@ibp.fhg.de)

HYPERION offers a generalization of Cremer's famous treatment [Akust. Z. 7, 81-104 (1942)] of the sound transmission of thin homogeneous plates in several respects: (i) periodically inhomogeneous plates, (ii) anisotropic bending stiffness, and (iii) different fluids on both sides of the plate. [A detailed account of the theory including some examples has been given in Acustica/acta acustica 84, 668-680 (1998).] The spatial periodicity generates propagating and nonpropagating diffracted waves, which may lead to several conspicuous features of the sound transmission loss curve. The most surprising phenomena are total reflection and total transmission below the critical frequency. These and other effects pertaining to refraction, near fields, and diffraction will be illustrated by an 8-min video film. Besides, it is worth mentioning that the computation of the transmission loss for diffuse-field excitation is rather time consuming, since a two-dimensional integration has to be performed over the two angles of incidence. The complexity of the integrand will be demonstrated graphically. [Financial support from the Deutsche Forschungsgemeinschaft is gratefully acknowledged.]
2:40

2pSAa3. A modal synthesis approach to investigate the influence of viscoelastic boundary conditions on the sound radiated from a plate backed cavity. Olivier Chiello, Franck C. Sgard (LASH, DGCB URA CNRS 1652, ENTPE-Rue Maurice Audin, 69518 Vaulx-en-velin Cedex, France, olivier.chiello@entpe.fr), and Noureddine Atalla (Univ. de Sherbrooke, QC J1K 2R1, Canada)

In this paper an efficient formulation is proposed to calculate the sound radiation from a plate with edges viscoelastically restrained against translation and rotation and coupled to a cavity. The viscoelastic constants can be varied arbitrarily along the plate boundary and reproduce simply supported, clamped, free or guided edges as limiting cases. The formulation classically couples a modal expansion for the structure in vacuo and the cavity, using a coordinate transformation to calculate the fluid-structure coupled modes. The modal basis associated with the structure in vacuo is determined using a free component mode synthesis technique considering the viscoelastic parameters as components with a residual flexibility only. This approach is appropriate to compute the coupled modes efficiently and to perform a parameter study. Numerical examples are presented in the case of a plate excited either mechanically or acoustically. The aim of the study is to determine which boundary parameters have an influence on the acoustic behavior of plates and to develop tools for the pratical design of plate mounting conditions.

3:00

2pSAa4. The structural acoustic optimization of an unstiffened cylinder finite-element model. Sergio De Rosa, Francesco Franco, Mauro Fontana, Romualdo Paino (Dept. of Aeronautical Eng., Univ. of Naples "Federico II," Via Claudio 21, 80125 Naples, Italy, derosa@unina.it), and Antonio Sollo (ALENIA Aerosp.-Aeronautic Div. Viale dell'Aeronautica, Pomigliano D'Arco (NA), Naples, Italy)

The presentation will show some results of an ongoing research, aimed to the tuning of a structural-acoustic optimization procedure. The target is the definition of a design tool in which it will be possible to include, at the same time, the weight and noise limits, together with the stress constraints, for getting reliable aircraft fuselage models. All the new capabilities included in the actual version of the MSC/NASTRAN have been tested. They allow analyzing the structural dynamics, the coupled acoustic response, to evaluate the structural-acoustic sensitivity and the global optimization process. Several similar attempts have been reported in the specific literature but, typically, different solvers have been used for each part of the problem (the structural degrees of freedom, the acoustic ones, and the optimization algorithm). Here, all results concern the application of the same finite-element model for a simplified (unstiffened) cylinder. The thicknesses of the plate elements have been grouped longitudinally and circumferentially, so that it has been possible to evaluate the predictive trends for fixed excitation frequency. Several optimization strategies have also been compared and discussed. They have also verified the optimized structural-acoustic models in output by evaluating the structural-acoustic geometric and dynamic coupling coefficients. 
2pSAa5. Finite element and boundary elements for underground input to SEA calculations. Philippe Jean (Centre Scientifque et Technique du Bâtiment, 24 rue Joseph Fourier, 38400 St. Martin d'Hères, France)

In order to study underground structures with a SEA model, special care must be given to ground loading which affects the behavior of the walls. The use of more precise techniques such as finite elements and boundary element techniques enables a description of complex phenomena and the computation of input to the SEA model. Therefore, several aspects have been analyzed: effect of type and position of source, type of ground, size and shape of the foundation, influence of the superstructure (size and type). Applications to the case of railway type situations are given. The source can be either on the surface or in the ground resulting in the generation of different types of waves.

3:40

2pSAa6. Radiation of sound from a monopole source near a freeflooded cylindrical shell. K. Steven Kim (Signatures Directorate, Carderock Div., Naval Surface Warfare Ctr., 9500 MacArthur Blvd., West Bethesda, MD 20817-5700)

Radiation of sound from an acoustic source changes if there is a structure nearby. Radiated and scattered acoustic pressure fields are obtained when a free-flooded cylindrical shell of finite length is subjected to acoustic waves from a monopole source. By solving integral equations formulated for an elastic thin shell with simply supported boundary conditions at both ends, far-field acoustic pressures are calculated. Cases of various source locations inside and outside a shell are discussed.

\section{4:00-4:20 Break}

\section{$4: 20$}

2pSAa7. Modeling structure-borne sound transmission via pipe systems to building structures. G. Susanne van der Jagt and Heiko J. Martin (Acoust. Lab., B-FAGO, Eindhoven Univ. of Technol., P.O. Box 513, 5600 MB Eindhoven, The Netherlands, g.s.v.d.jagt@bwk.tue.nl)

In residential buildings many people are annoyed by noise caused by their neighbors, including structure-borne sound caused by drinking water, sanitary, and other installations. In different building regulations, limits are set to the maximum allowable sound level caused by installations in a room. However, predictions of the sound level in the design stage are not possible. In this research, a framework for structure-borne sound transmission models for pipe systems, including source characterization, has been developed. Further, the application possibilities of existing calculation methods in the quantification of the sound transmission have been investigated. For example, the application possibilities of the finite-element method (FEM) and statistical energy analysis (SEA) depend on the element type, including dimensions and material (pipe, mounting, or building structure), the wave type, and the frequency area. By combining both methods, calculations for the whole audible frequency area seem possible. Some models are presented, together with calculated values of characteristic parameters. Measurements can be used to complete and validate the models, for example by using a measurement setup based on the plate method. The interaction between calculations and measurements will form the basis for future research. The future research setup is also presented.

\section{4:40}

2pSAa8. Estimating radiation modes for broadband measurement. Scott D. Sommerfeldt and Dong Lin (Dept. of Phys., Brigham Young Univ., Provo, UT 84602-4673, s_sommerfeldt@byu.edu)

Acoustic radiation modes have been investigated as a means of estimating far-field radiated acoustic power, based on structural vibration measurements. One of the difficulties associated with radiation modes is the fact that the mode shapes are frequency dependent. Much of the research in the past associated with radiation modes has focused on the low-frequency range, where the radiation modes are nearly frequency independent. The work reported here is aimed at developing a measurement approach based on radiation modes, which is applicable in the low- and mid-frequency range, where the frequency dependence of the radiation modes cannot be ignored. In general, if a limited number of point vibration sensors are to be used to estimate the radiated acoustic power, the appropriate locations on the structure for those sensors will change for each frequency. However, it will be shown that if one uses distributed sensors, it is possible to use a single sensor configuration to estimate the acoustic radiation over a broad range of frequencies. Numerical results will be shown that indicate the measurement accuracy that can be expected over a range of frequencies up to a normalized frequency of $k L=15$.

\section{5:00}

2pSAa9. Integral formulations for the vibroacoustic characterization of a cello. C. Langrenne and A. Garcia (Laboratoire d'Acoust. du CNAM, 5 rue du Vertbois, 75003 Paris, France)

An isoparametric element formulation is used for implementing the Helmholtz integral formulation associated with arbitrary shaped threedimensional bodies [M. Bonnet, Eyrolles editions, 1997]. The inverse problem is solved, i.e., the normal velocity distribution is determined on the surface of the instrument. In this way, the direct problem can be solved: the acoustic field can be calculated anywhere (near field, far field). Because of difficulties in solving the inverse problem (ill-posed problem, i.e., of which the solution is very sensitive to the errors of measurements), a regularization method is used to find the nearest solution of the real one [C. Langrenne and A. Garcia, Inter-Noise 97 Proc., Hungary, August 25-27 (1997)]. Experimental vibroacoustic characterization is done from holography measurements, without any contact with the stucture of the instrument, with the help of a robot describing a cylindrical surface.

\section{$5: 20$}

2pSAa10. Effect of internal inhomogeneities on acoustic scattering from finite cylindrical shell bounded by hemispherical endcaps. Nicolas Touraine, Dominique Décultot, Gérard Maze (Lab. Acoust. Ultrasonore et Electron. (LAUE), UPRESA CNRS 6068, Université du Havre, Pl. Robert Schuman, 76610 Le Havre, France), Aleksander Klauson, and Jaan Metsaveer (Tallinn Tech. Univ., EE 0026 Tallinn, Estonia)

The acoustic scattering from an air-filled finite cylindrical shell with hemispherical endcaps is considered. In axial incidence, experimental studies have shown formation of compressional wave $S 0$ resonances. These resonances are predicted and identified by applying the phasematching condition over the meridian circumference. However, frequency irregularities observed experimentally on spectra have suggested that the assemblage quality during the manufacturing of the object may be not negligible. Indeed, internal nonuniformities of the shell at the junctions of cylindrical and hemispherical shells are present and modify certain wave propagation phenomena. The aim of this study is to discover these experimental conditions of the studied object through a numerical model with internal annular inhomogeneities localized at the junctions of the substructures and for two different $b / a$ ratios (internal to external radius ratio) equal to 0.97 and 0.99 . In the studied frequency range $(50-300 \mathrm{kHz})$, in addition to the $S 0$ wave, another peripheral wave $A$ can be generated for the ratio $b / a=0.97$. The numerical approach is based on the discretization of the shell and determination of its eigenmodes (FEM). Acoustical pressure field is found by the boundary integral equation procedure (BEM). The comparison of numerical and experimental results is presented in frequency and time domains. 
2pSAa11. Designing structures for noise and vibration control with statistical energy analysis. Liangyu Huang and P. K. Raju (Dept. of Mech. Eng., Auburn Univ., Auburn, AL 36849)

Statistical energy analysis (SEA) was initially developed for predicting noise and vibration levels in interior spaces and structures at audio frequencies. The methodology developed in this paper extends traditional statistical energy analysis beyond the prediction of mean-rms vibroacoustic response. An SEA response sensitivity algorithm was formulated to relate variability of design parameters to SEA response variation. The method can be used to sort the design parameters which have the greatest effect on the sound-pressure levels in acoustic spaces. The SEA response variance analysis relates response variance directly to variances in design parameters. This methodology is important for acoustic design of aircraft, ship, and automotive vehicles because it enables the user to qualitatively and quantitatively determine the rank and variations of vehicle interior sound-pressure level with respect to changes in design parameters. When fully developed, this methodology can be a powerful tool for identifying the design changes that would be most effective for controlling noise and vibration from structures.
2pSAa12. The acoustical power radiated by a planar annular plate for the form of vibrations $(\mathbf{0 , n})$. W. P. Rdzanek (Inst. of Phys., Pedagogical Univ. of Rzeszów, Rejtana 16a, 35-311 Rzeszów, Poland) and Z. Engel (Mining-Metallurgical Acad., Al. Mickiewicza 30, 30-059 Kraków, Poland)

The acoustic power radiated by a thin annular plate fixed rigidly, with both internal/external edges, into a planar rigid infinite baffle is analyzed. These processes are considered sinusoidal in time. It is assumed that the annular plate vibrates axially symmetric. The radiated acoustic power integral formula is transformed with the introduction of the contour integral at the plane of complex variable. Then the Cauchy theorem about residua is used. An elementary, useful for calculations in case of high frequencies, form of the formula of the radiated acoustic wave is obtained. This formula represents the acoustic power with the assumption that the annular plate vibrates with the $n$th axially symmetric mode.

\title{
Session 2pSAb
}

\section{International Workshop on Active Noise and Vibration Control Structural Acoustics and Vibration: Active Noise Control in Ducts}

\author{
Scott D. Sommerfeldt, Cochair \\ Department of Physics and Astronomy, Brigham Young University, Provo, Utah 84602-4673, USA \\ Gérard Mangiante, Cochair \\ Laboratoire de Mechanique et d'Acoustique, 31 Chemin Joseph-Aiguier, 13402 Marseille, Cedex 20, France
}

Invited Papers

2:00

2pSAb1. Development of an active exhaust silencer for combustion engines. Rene Boonen and Paul Sas (KU Leuven, Dept. of Mech. Eng., PMA, Celestijnenlaan 300B, B-3001 Heverlee, Belgium, rene.boonen@mech.kuleuven.ac.be)

A silencer is developed to attenuate engine exhaust noise using active control. The device consists of an electrically driven valve, combined with a buffer volume. Using the mean flow through the valve and the pressure fluctuations in the volume, the valve regulates the flow such that only the mean flow passes the tail pipe outlet. The flow fluctuations are temporally buffered in the volume. A cold engine simulator is developed to experiment with exhaust systems. This device generates realistic exhaust noise and the matching gas flow using compressed air. It allows quick and reliable acoustic and fluid dynamic experiments on exhaust prototypes. The valve is controlled using a nonadaptive feed-forward algorithm. The active silencer reduces the exhaust noise $13 \mathrm{dBA}$. However, the nonlinear relation between valve stroke and pressure drop limits the performance of the controller. Currently, a feedback controller is being implemented. It improves the performance, but is now limited by the time delay between control valve and the error pressure sensor. Future developments are now focused to compensate the time delay, using a Smith compensator, or repetitive control. Adaptive feed-forward is in consideration also, but this probably will not be ready when the workshop is held. [Work supported by BOSAL International.]

2pSAb2. Effects of geometric discontinuities and filter lengths on active control in ducts. Denis Duhamel (ENPC-CERAM, 6 et 8 Ave. Blaise Pascal, Cité Descartes, Champs-sur-Marne, 77455 Marne-la-Vallée Cedex 2, France) and Philippe Sergent (STCPVMN, 60321 Compiègne Cedex, France)

The active control is often used to reduce noise propagation in ducts, usually in straight sections. However, industrial ducts often contain discontinuities where the secondary source of an active system can or must be placed. The possibility of active control in such situations is studied and compared to the case of a straight duct. It is shown that a correct position for the secondary source allows one to take advantage of both passive and active attenuations, and can improve the global system. For a right angle or a resonator, simple analytical formulas can be obtained for the active attenuation, and it can be proved that the power output of the secondary source is 
null. The influence of other important parameters such as the number of coefficients of the FIR filter in a filtered-X LMS algorithm, and the sampling frequency used for the control or the loss factor in the duct, are also estimated by analytical formulas. This gives, for instance, explanations for the frequency bands over which the control efficiency is low. In each case, comparisons with experimental data are provided and they support the theoretical conclusions quite well.

\section{Contributed Papers}

\section{2:40}

2pSAb3. An active silencer for harsh environmental conditions. Jan Krüger (Fraunhofer-Inst. Bauphysik Stuttgart (IBP), Stuttgart Nobelstrasse 12, D-70569 Stuttgart, Germany)

Active noise control has proven effective in the reduction of lowfrequency noise in ducts, e.g., in air conditioning systems. However, in many applications the capability of proper operation of an active silencer is severely limited by the harsh environment. In fact, higher temperatures, static and dynamic pressure loads, extreme sound power levels, and abrasive flow often prohibit the use of active silencers at all. Appropriate design details for an actively absorbing silencer are presented, leading to a new construction with high performance and durability. Using standard components (microphone, loudspeaker), robust protection measures are developed which can be adapted to particular applications. A computational model derived from the theory of active silencers allows the variation of design parameters and the prediction of the insertion loss. To verify the numerical results, experiments under several acoustical and thermal conditions were conducted.

\section{3:00}

2pSAb4. Active noise control of higher-order modes in a rectangular duct using stabilized fast transversal filters. Holger Opfer and Dieter Guicking (Drittes Physikalisches Institut, Universitaet Goettingen, Buergerstr. 42-44, 37073 Goettingen, Germany, holger@physik3.gwdg.de)

For active noise control of sound propagating down a duct in the fundamental mode commercial systems are available. Cancellation of sound also propagating in higher-order modes adds the requirement of higher computational power for the signal processing unit, and stability problems due to source coupling effects. The widely used LMS algorithms need little computational effort, are very stable, but also have a slow tracking behavior to changes in the input signal. Depending on the eigenvalue spread of the input signal's autocorrelation matrix they lead to slow convergence. One method to overcome this problem is to use stabilized fast RLS algorithms which are independent of the statistics of the input signal. A multi-channel stabilized fast transversal filter algorithm has been investigated experimentally in a rectangular duct with respect to its stability, convergence, and performance.

\section{3:20}

2pSAb5. A hybrid active-passive system for controlling locomotive exhaust noise. Paul J. Remington (BBN Technol., 70 Fawcett St., Cambridge, MA 02138), Scott Knight, and Douglas Hanna (BBN Technologies, New London, CT 06320-6147)

Diesel electric locomotives represent the primary source of motive power in the U.S. railroad industry, and are a significant source of environmental noise. There is a variety of noise sources on a locomotive; however, two sources will have to be controlled before significant reductions in locomotive noise can be achieved: diesel engine exhaust noise and cooling fan noise. Here a system is described that uses both active and passive components to control broadband exhaust noise below $4 \mathrm{kHz}$. The active system utilizes eight actuators and eight residual microphones in an adaptive feed-forward configuration to control tonal noise below $200 \mathrm{~Hz}$. The passive system is a compact exhaust silencer designed to control exhaust noise above that frequency while still fitting within the limited space available beneath the locomotive hood. Both active and passive systems will be described in detail, and practical problems encountered in the design of the combined systems will be described. Performance estimates for both the active and passive system will be presented along with the available measurements. [This work was supported under contract to the U.S. Federal Railroad Administration.]

\section{3:40-4:00 Break}

\section{4:00}

2pSAb6. Active control of pulsating flows in transitory conditions. J. Tartarin and F. Carimantran (Lab. d'Etudes Aerodynamiques, Univ. de Poitiers, bat. H, 40 avenue du Recteur Pineau, 86022 Poitiers, France, Francois.Carrimantran@lea.univ-poitiers.fr)

The active control in a duct in the presence of hot and/or corrosive gasses (e.g., exhaust system) or even heavy fluids (oil, fuel, water, ...) needs an actuator that is particularly resistant. That's why the LEA designed an original auxiliary source [Hardouin et al., Acta Acustica 1, 189-198 (1993)]: an oscillating flap which acts in a flow as an aeroacoustic source. Its high efficiency in very low frequencies associated with its little bulk is also another major interest. For active control, because it is a source connected in series with the primary source, an online identification is preferable for better adaptive character. Therefore specific algorithms were developed in frequency domain for command strategies of feedback synchronous type. Other time-frequency domain algorithms were implemented. In view of industrial applications, these different algorithms were experimentally compared, particularly in transitory conditions. Jointly, simulations were led to allow the development and the optimization of exhaust mufflers including the flap and passive devices.

\section{4:20}

2pSAb7. Experimental investigations on active suppression of sound propagation in a fluid-filled pipe. Roland Lippold and Joachim Scheuren (Müller-BBM GmbH, Robert-Koch-Straße 11, D-82152 Planegg, Germany, li@mbbm.de)

The control of sound propagating along fluid-filled pipes is complicated because of the strong coupling between the structural and the fluid transmission path. If the propagation along the structural path is supressed (e.g., by passive compensation in the case presented), it is possible to reduce the propagating sound by active means only acting on the fluid. This statement was proved by several experiments. The experimental setup consisted of one pipe made of aluminum (length $4 \mathrm{~m}$, wall thickness $5 \mathrm{~mm}$, inner diameter $90 \mathrm{~mm})$ and two pipes made of steel $(3 \mathrm{~m} / 1.5 \mathrm{~m}$, $2.5 \mathrm{~mm}, 70 \mathrm{~mm}$ ) which were connected via 90-deg bends and filled with water. For excitation and compensation of sound in the fluid, shaker driven pistons acting perpendicular to the pipe axes were used. The frequency of the harmonic excitation in the frequency range from 600 up to $800 \mathrm{~Hz}$ was known to the compensation algorithm. If the passive compensation of the structural path was sufficient, the reductions of sound pressure in the fluid achieved at and behind the error sensor were between 10 and $20 \mathrm{~dB}$. Otherwise, reductions of the same order were locally restricted to the position of the error sensor.

\section{4:40}

2pSAb8. Automobile gas-exchange systems low-frequency noise reduction using active noise control units. Andrei V. Vassiliev (Dept. of Labour and Environ. Protection, Togliatti Polytechnic Inst., Belorusskaya str. 14, Togliatti, 445667 Russia)

The main automobile aerodynamic noise sources radiating into the environment are internal combustion engine gas-exchange systems (intake and exhaust). Practical possibilities of active noise control unit applica- 
tions for automobile gas-exchange system low-frequency noise reduction are discussed. Existing technical constructions of active noise control units (including those patented by author) for automobile intake and exhaust noise reduction are concerned. New construction of a unit is proposed. Its peculiarity is joint intake and exhaust noise reduction possibility. The signal of engine rotation frequency pick-up is input into the control system through the low-pass filter and transducer and then transmitted by the loudspeakers through the special waveguides into the zones of compensation near the intake and exhaust pipe open ends. Throttle opening angle is accounted for. Calculative results show the efficiency of such a construction operation.

TUESDAY AFTERNOON, 16 MARCH 1999

ROOM H3010, 2:00 TO 6:20 P.M.

\title{
Session 2pSCa
}

\section{Speech Communication: Data Based Speech Synthesis and Analysis III}

\author{
Juergen Schroeter, Chair \\ AT\&T Laboratories-Research, 180 Park Avenue, Building 103, Florham Park, New Jersey 07932-0971, USA
}

\section{Contributed Papers}

\section{2:00}

2pSCa1. VCV synthesis from muscle commands. Perrier Pascal (ICP-INPG, 46 Av. Felix Viallet, 38031 Grenoble Cedex, France), Yohan Payan (TIMC, IMAG, 38706 La Tronche, France), Joseph Perkell, Majid Zandipour, and Melanie Matthies (MIT, Cambridge, MA 02139)

The biomechanical model of the tongue developed by Payan and Perrier [Speech Commun. 22, 185-205 (1997)] is used to generate tongue movements in VCV sequences, where $\mathrm{V}$ is $[\mathrm{i}]$, [a], or $[\mathrm{u}]$ and $\mathrm{C}$ is the stop consonant $[\mathrm{k}]$. Tissues' elastic properties are accounted for in finiteelement modeling, and the mechanisms underlying the production of muscle forces are modeled according to Feldman's Equilibrium Point Hypothesis. Each elementary sound is associated with a target that is described as a static equilibrium position of the tongue, which is likely to vary with the phonemic context. In vowel production, the target is actually supposed to be reached, while the production of stop consonants consists of movements toward virtual targets located beyond the palate, which therefore cannot be reached. The collision of the tongue against the palate is modeled according to a penalty method, based on a nonlinear relationship between contact force and position/velocity of points located on the tongue surface [Marhefka and Orin, IEEE Conf. Robotics and Automation, 1662-1668 (1996)]. The acoustic signal is generated with a onedimensional low-frequency approximation based on the Kelly-Lochbaum model. Synthetic kinematic and acoustic signals are compared with human speakers' data. [Work supported by CNRS, NSF, and NIH.]

\section{2:20}

2pSCa2. Speech synthesis of VCV sequence using a physiological articulatory model. Jianwu Dang (ATR Human Information Res. Labs., 2-2 Hikaridai, Seikacho Soraku-gun, Kyoto, 619-0288 Japan and Univ. of Waterloo, Waterloo, ON N2L 3G1, Canada, jwdang@crg3.uwaterloo.ca) and Kiyoshi Honda (ATR Human Information Res. Labs., 2-2 Hikaridai, Seikacho Soraku-gun, Kyoto, 619-0288 Japan)

A 3-D articulatory model has been constructed based on volumetric MR images for a Japanese male speaker, which is driven by a targetdependent control strategy to produce dynamic articulation for vowel sequences [Dang and Honda, ICSLP98]. This work describes a multi-point control strategy that is developed for generating vowel-consonant-vowel (VCV) sequence. Three control points are located on the mandible, tongue tip, and tongue dorsum, and they are operated in each muscle workspace. Contributions of the tongue and jaw muscles to each control point are evaluated by model simulation, and described as a weighted coefficient. Muscle activation signals are determined according to the articulatory vector from the current position to the target. The total activation pattern of the muscles is the sum of the activation signals calculated for the control points. Dynamic vocal tract shape is obtained by using the activation pattern to excite the model, and compared with MRI movies of VCV sequence for the same speaker [Masaki and Honda, J. Acoust. Soc. Am. 102, 3166(A) (1997)]. The VCV sequence is synthesized from a set of vocal tact area functions of the model, based on vocal tract widths in the midsagittal and parasagittal planes, and compared with real speech.

\section{2:40}

2pSCa3. The FUL speech recognition system. Aditi Lahiri and Henning Reetz (Dept. of Linguist., Univ. of Konstanz, D186, 78462 Konstanz, Germany, aditi.lahiri@uni-konstanz.de)

The featurally underspecified lexicon (FUL) speech recognition system is based on a lexicon where words are represented by radically underspecified phonological features. Features are extracted from the speech stream and are mapped with a ternary logic (match, no-mismatch, mismatch) to the entries in the 100000 -word lexicon without building a segmental representation. The mapping process operates from left to right and allows for certain insertions and deletions of segments of the words in the lexicon. Mismatching features are deactivated from the search. Word candidates remaining after this selection undergo the parallel active prosodic and syntactic parsing of phrase hypothesis. The system is speaker independent and not sensitive to microphone or line conditions and does not need a high signal-to-noise ratio.

\section{3:00}

2pSCa4. Parameter estimation from speech signals for tube models. Karl Schnell and Arild Lacroix (Institut für Angewandte Physik, Johann Wolfgang Goethe Univ., D-60054 Frankfurt am Main, Germany)

Discrete time tube models implemented by lattice structures can be used to simulate the propagation of sound waves in the vocal tract. To cover a wide variety of speech sounds, it is necessary to extend the standard all-pole tube model by coupling the nasal tract, and, furthermore, enable the excitation of the tube system at different places in between. The resulting filter structure $H(z)$ now shows poles as well as zeros in the transfer function. The impedance of the lip opening is realized using a model from Laine [U. K. Laine, "Modeling of lip radiation impedance in the $z$-domain," Proc. ICASSP-82, 1992-1995 (1982)], which includes one pole and one zero in the $z$-plane. The parameters of the tube model will then be estimated from speech by minimization of an error, which describes the spectral distance between $H(z)$ and the prefiltered speech signal. The prefilter is used for the separation of signal components due to excitation and radiation. A gradient-based optimization is implemented to find the minimum. Following this procedure, the frequency response is able to approximate the spectral envelope of the speech signal. The resulting areas correspond to those obtained by $\mathrm{x}$-ray or NMR investigations. 
directly. This seems to be less prone to produce "musical noise." The dominant common MF (mostly corresponding to $F_{0}$ ) is enhanced by an

2pSCa5. A preliminary study of speech transformation using empirically defined articulatory modes. Brad Story and Ingo Titze (WJ Gould Voice Res. Ctr., Denver Ctr. for the Performing Arts, 1245 Champa St., Denver, CO 80204)

In previous work it has been shown that a speaker-specific set of vocal tract shapes (acquired using MRI) corresponding to vowels can be decomposed into orthogonal components or "modes," and effectively parameterized by the modal coefficients. Furthermore, a nearly one-to-one mapping was found to exist between the modal coefficients of the two most significant modes and the $F 1-F 2$ formant space. Thus an inverse mapping of time-varying formants extracted from recorded speech back to modal coefficients (and consequently to vocal tract area functions) was made possible; the derived sequence of area functions can be used in a simulation of the original speech utterance. In this study, it is first shown that similar orthogonal modes exist for three additional speakers. It will then be demonstrated that the time-varying modal coefficients determined for a recorded sentence via the inverse mapping of one speaker can also be used to create a sequence of area functions based on one of the other speaker's vocal tracts. A subsequent simulation produces the original sentence but with the vocal tract characteristics of the new speaker. The results also suggest that the time-varying modal coefficients may define common gestures across speakers even though their acoustic characteristics can be different.

\section{3:40}

2pSCa6. A neural model for auditory scene analysis. Dekun Yang, Georg F. Meyer, and William A. Ainsworth (Dept. of Commun. and Neurosci., Keele Univ., Keele, Staffs ST5 5BG, UK, dekun@cs.keele.ac.uk)

The fundamental process of auditory scene analysis is the organization of elementary acoustic features in a complex auditory scene into grouped meaningful auditory streams. There are two important issues which need to be addressed for modeling auditory scene analysis. The first issue is concerned with the representation of elementary acoustic features, whilst the second issue is related to the binding mechanism. This paper presents a neural model for auditory scene analysis in which a two-dimensional amplitude modulation (AM) map is used to represent elementary acoustic features and the synchronization of neural oscillators is adopted as the binding mechanism. The AM map captures the modulation frequencies of sound signals filtered by an auditory filterbank. Since the modulation frequencies are the $F 0$-related features for voiced speech signals, $F 0$-based segregation can be utilized to group the auditory streams. The grouping of $F 0$-related features is attained as the formation of the synchronization of nonlinear neural oscillators. Each oscillator is associated with a certain modulation frequency. A set of oscillators are synchronized only when their associated frequencies are harmonically related. The proposed model is tested on synthetic double-vowel identification and the results are in accordance with psychophysical data.

\section{4:00-4:20 Break}

\section{$4: 20$}

2pSCa7. Noise reduction for speech signals by operations on the modulation frequency spectrum. Hans Werner Strube and Hans Wilmers (Drittes Physikalisches Inst., Univ. Göttingen, D-37073 Göttingen, Germany)

A new method is presented for enhancing the contrast between speech and background noise, which might be useful as a preprocessor for speech recognizers or even for improving the audible speech signal. As in the brain a representation of the acoustic modulation spectrum exists, it is suggested to use similar methods for signal separation. Previous models have shown the possibility of selecting frequency channels based on modulation frequency (MF) analysis, if the signals to be separated have sufficiently different modulations. Instead of selecting or weighting frequency channels, in the present approach the MF spectrum is processed adaptive bandpass filter in each frequency channel, and the low-MF components are artificially reconstructed before transforming back. No explicit $F_{0}$ measurement is required. Nonstationary sounds (detected by the width of the low-MF part) are specially treated, for instance, by channeldependent mixing with the original signal. The method is tested with a HMM recognizer (at Univ. Oldenburg) and compared to classic methods such as spectral subtraction. Intelligibility measurements are planned. Examples of processed speech will be presented.

\section{$4: 40$}

2pSCa8. Resolution capability of reassigned Fourier spectrum. Dekun Yang, William A. Ainsworth, and Georg F. Meyer (Dept. of Commun. and Neurosci., Keele Univ., Keele, Staffs ST5 5BG, UK, dekun@cs.keele.ac.uk)

High-resolution spectral estimation is important for speech analysis. The conventional Fourier transform technique cannot offer sufficient resolution for speech signals of short durations. The reassignment method has been recently developed to improve the resolution of the Fourier spectrum. The basic idea of the reassignment method is to assign the value of the Fourier spectrum to the gravity center of the region rather than the geometric center of the region. However, the characteristics of the reassigned Fourier spectrum have not been fully understood. This paper is concerned with a detailed investigation of the resolution capability of the reassigned Fourier spectrum. The factors affecting resolution, such as frequency separation and relative amplitude, are studied. The minimal frequency separation which can be resolved in the reassigned Fourier spectrum is determined. As a result, it shows that the reassigned Fourier spectrum has better resolution capability in comparison to the Fourier spectrum. Furthermore, the study shows that the frequency estimator of closely spaced sinusoids has a bias. The bias is due to the interaction of the two sinusoids under the assignment operation. The expression of the bias is derived and shows that the bias can be ignored for most speech signals.

\section{5:00}

2pSCa9. Improved spectral resolution via the reassigned Fourier transform. Charles R. Day, William A. Ainsworth, and Georg F. Meyer (Dept. of Commun. \& Neurosci., Keele Univ., Keele, Staffordshire ST5 5BG, UK, charles@cs.keele.ac.uk)

A modified form of the Fourier transform, the reassigned Fourier transform (RAFT), uses phase as well as magnitude information. Its properties are shown to be superior for both pure-tone and speech signal analysis. The RAFT technique has the potential to deliver higher resolution spectrograms than the fast Fourier transform (FFT) for a given signal analysis window. The reassignment of energy with respect to both time and frequency, such that the reassignments model the time-frequency fluctuations of the sampled signal, allows the RAFT to deliver higher resolution than the FFT (with the FFT, spectral/temporal analyses place all of the energy at points lying in the center of either a time/frequency sample-window, respectively). In particular, the improved spectral resolution of the RAFT for a given signal analysis window size is compared with that of the FFT for a fundamental speech processing application: pitch tracking. The results indicate that use of the RAFT instead of the FFT allows shorter signal analysis windows to be used. The pitch tracking performance of the RAFT with short analysis windows (e.g., 25 and 12 $\mathrm{ms}$ ) is better than the FFT. [Work supported by the UK's EPSRC.]

\section{$5: 20$}

2pSCa10. Intelligibility enhancement of synthetic speech heard via telephone in a noisy environment. Stefanie Köster and Joachim Mersdorf (Inst. fuer Kommunikationsakustik, Ruhr-Univ. Bochum, Universitätsstr. 150, D-44780 Bochum, Germany)

The intelligibility of synthetic speech, like the intelligibility of natural speech, decreases if it is transmitted via telephone and/or heard in a noisy environment. A natural speaker adapts his speaking style to these hard 
speaking conditions in order to restore intelligibility. With new applications for speech synthesis, e.g., navigation systems for cars, the need for adapting synthetic speech to hard speaking conditions arises. Here, the results of intelligibility tests for German databased synthetic speech in comparison to natural speech are presented, and first methods of intelligibility enhancement for synthetic speech are given. It is shown that under the conditions mentioned above unadapted synthetic speech has a lower segmental intelligibility for all phonemes or phoneme clusters than natural speech. For that reason the methods applied affected all speech sounds equally. They are based on prevoius studies of natural speech produced under stress conditions like a noise afflicted environment [Summers et al., J. Acoust. Soc. Am. 84, 917-928 (1988)]. It is shown that the intelligibility of synthetic speech is improved significantly by these enhancement methods. The results give evidence to an individual adaptation for phonemes or phoneme clusters in future.

\section{$5: 40$}

2pSCa11. Query language for access to speech corpora. Andreas Mengel and Ulrich Heid (Inst. of Natural Lang. Processing, Stuttgart Univ., Azenbergstrasse 12, 70174 Stuttgart, Germany, mengel@ims.uni-stuttgart.de)

Typically, speech corpora are designed for the study of speech, its patterns, and their influences. Corpora for synthesis support the extraction and reuse of units in concatenative speech synthesis systems. Recent approaches consider speech segments of variable length, to enhance naturalness. The development of spoken language dialog systems (SLDSs) and similar applications requires dialog corpora with multiple information, such as prosodic labeling, grammatical annotations, dialog acts, etc. For constructing such multilevel corpora and, particularly, for retrieving useful information from there, three aspects are crucial: A theory for the description of the corpora, an effective markup and methods for specifying, and efficiently retrieving relevant portions of the data. MATE (EU Telematics Project LE4-8370) proposes standards for an integrated and consistent multilevel annotation based on the existing TEI standard (Text Encoding Initiative). For the access to massively marked-up speech data, a query language and a query engine have been developed. This allows the retrieval of any combination of data, with linguistic annotations from different levels. The presentation will describe and demonstrate the query language.

\section{6:00}

2pSCa12. Prosody templates in word-level synthesis. Frode Holm and Kazue Hata (Speech Technol. Lab., 3888 State St., Santa Barbara, CA 93105)

This paper describes an approach to word-level prosody with the goal of achieving natural-sounding human intonation. This study comprised about 3000 words in sentence initial position, uttered by a female native speaker of American English. From these recordings general $F 0$ and duration templates were extracted, initially based on stress-pattern alone. Results with regards to $F 0$ templates [F. Holm and K. Hata, ICSLP (1998)] had previously been reported, which showed great promise for a practical synthesis implementation. In this paper duration templates will be focused on. Obtaining temporal prosody patterns is a much harder problem than it is for $F 0$ contours. This is largely due to the fact that one cannot separate a high-level prosodic intent from purely articulatory constraints merely by examining individual segmental data. This methodology relies on a concept of stretchability, which gives a clear measure of how much a given cluster of segments can change its duration in natural speech. The higher this measure is, the more of the speaker's intentional temporal adjustment is expected to be found in that cluster. Using this concept, a template-generating procedure has been formulated that achieves a very high prediction rate of a speaker's duration patterns.

\title{
Session 2pSCb
}

\section{Speech Communication: Cross Language and L2 Processing II (Poster Session)}

\author{
Winifred Strange, Chair \\ City University of New York, 33 West 42 Street, New York, New York 10036, USA
}

\section{Contributed Papers}

\begin{abstract}
All posters will be on display in the Poster Gallery from Monday to Wednesday, 15-17 March. Authors will be present at their posters on Tuesday, 16 March. To allow contributors an opportunity to see other posters, contributors of odd-numbered papers will be at their posters from 2:00 p.m. to 4:00 p.m. and contributors of even-numbered papers will be at their posters from 4:00 p.m. to 6:00 p.m.
\end{abstract}

\begin{abstract}
2pSCb1. The influence of vowel context on the Japanese listener's identification of English voiceless fricatives. William L. Martens and Stephen Lambacher (Univ. of Aizu, Tsuruga, Ikki-machi, Aizu-Wakamatsu City, Fukushima, 965-8580 Japan)
\end{abstract}

In order to examine the influence of vowel context on the ability of native Japanese speakers to distinguish between English voiceless fricatives, recognition rates from a five-alternative, forced-choice (5AFC) test were analyzed in terms of receiver operating characteristics. This signaldetection-theoretic analysis showed contrasting effects of vowel context on sensitivity versus response bias. Subjects heard each of 75 nonsense syllables spoken by three native speakers of English, and were asked to report whether the syllable they heard contained $/ \mathrm{f} /, / \mathrm{s} /, / \mathrm{f} /, / \theta /$, or $/ \mathrm{h} /$. Fricative identifiability (measured by the index of sensitivity, $d^{\prime}$ ) appeared to be modulated primarily by phonemic and phonetic factors. In contrast, the likelihood of giving one fricative response over another without regard for which fricative is presented (measured by the index of bias, $\beta$ ) appeared to be modulated by more subjective factors such as familiarity with common loan words (words of foreign origin). A cross-linguistic examination of phonemic and phonetic factors revealed possible reasons why the Japanese listener's identification of English fricatives is considerably poorer in particular vowel contexts, and how differences in fricative production can make it more difficult to make certain phonetic distinctions. 
2pSCb2. Can English listeners learning Japanese as a second language employ a mora-based segmentation procedure? Takashi Otake (Dept. of English, Dokkyo Univ., 1-1 Gakuen-cho, Soka, Saitama, 340 Japan, otake@dokkyo.ac.jp) and Kiyoko Yoneyama (Ohio State Univ., Columbus, OH 43210)

Previous research has shown that monolingual listeners exploit language-specific segmentation routines, based on linguistic rhythm, not only with native but also with foreign input. Thus monolingual Japanese listeners used a mora-based segmentation procedure with both Japanese and English, while monolingual English listeners, whose linguistic rhythm is different from Japanese, did not use this procedure [Cutler and Otake, JML 33, 824-844 (1994)]. Bilinguals, however, can inhibit an inappropriate rhythm-based segmentation procedure [Cutler et al., Cogn. Psychol. 24, 381-410 (1992)]. The present study explored whether English listeners learning Japanese as a second language could employ a mora-based segmentation procedure in Japanese. Three groups of American subjects, whose Japanese proficiency differed, participated in the experiment, using the phoneme monitoring materials of Cutler and Otake (1994). The prediction based on the findings above was that none of these subjects would be sensitive to morae in listening to Japanese. The results, however, showed that all the subject groups were consistently sensitive to morae, which suggests that English listeners can employ a non-native segmentation procedure in a foreign input as long as they have at least begun to acquire the foreign phonology. [Work supported by Fulbright senior research grant and the Telecommunications Advancement Foundation.]

2pSCb3. Intonational foreign accent in the speech of American speakers of German. Matthias Jilka (Inst. of Natural Lang. Processing, Univ. of Stuttgart, Azenbergstr. 12, 70174 Stuttgart, Germany)

The $F 0$ patterns typical of German and American English are analyzed within the framework of a category-based description of intonation events as established by the ToBI prosodic transcription system. This description is supplemented by intonation rules for the categories' phonetic implementation. The rules are also the basis for the process of $F 0$ generation and resynthesis, which facilitates determining which aspects in non-native speech are responsible for the perception of intonational foreign accent. An analysis of the productions of native speakers of American English who are fluent in German shows a distinct influence of L1 prosodic characteristics on L2. It consists of a transfer of native prosodic settings to L2. Several types of intonational foreign accent are identified. Tonal categories, such as pitch accents or boundary tones, are affected in two ways: either an incorrect category is selected or it is assigned to an inappropriate part of the intonation phrase. Transfer effects are particularly distinct in the American speakers' phonetic realization of corresponding tonal categories. The Americans also maintain other prosodic language-specific features like pitch range. Overall, prosodic behavior in L2 acquisition seems quite similar to segmental behavior and in accordance with current models of segmental L2 acquisition.

2pSCb4. A study of the language dominance effects on bilinguals' voice quality. Marielle Bruyninckx and Bernard Harmegnies (Phonet. Lab Pl. du Parc, 18 B-7000 Mons, France,

Marielle.Bruyninckx@umh.ac.be )

This paper examines whether language dominance exerts influences on speaker's voice quality. Ten French-Polish bilingual subjects, split into a French-dominant group and a Polish-dominant group, were selected. They were asked to record five utterances of two balanced texts (one for each language). One FFT-based LTAS was computed for each utterance. The so-obtained 100 spectra were compared by means of a pattern recognition procedure: the SDDD dissimilarity index. Two types of within-speaker comparisons were performed for each subject: within-language comparisons and between-language comparisons. The results show that the average SDDD index values derived from the between-language comparisons score higher than those drawn from within-language matchings. This confirms our earlier results based on different types of bilinguals. The withinlanguage FR/FR SDDD index values tend to score higher in the Frenchdominant group than the POL/POL ones; the opposite relationship can be observed in Polish-dominant subjects. These results empower one to generalize the idea that language dominance seems to be a significant variable in the characterization of the phonetic behavior of speakers using more than one language.

2pSCb5. Effects of $\mathrm{L1}$ phonotactic constraints on L2 speech perception and production. Satoshi Imaizumi, Yuji Tamekawa (Dept. of Speech and Cognit. Sci., Grad. School of Medicine, Univ. of Tokyo, Bunkyo, Tokyo, 113-0033 Japan), Hidemi Itoh (Tohoku Univ., Sendai, 980-8575 Japan), Toshisada Deguchi (Tokyo Gakugei Univ., Tokyo, 184-8501 Japan), and Koichi Mori (Res. Inst. Natl. Rehabilitation Ctr. for the Disabled, Saitama, 359-8555 Japan)

Effects of L1 phonotactic constraints on L2 speech perception and production were analyzed during the course of audio-visual perceptual training for Japanese adult learners of English by observing articulation of words containing $/ 1 /, / r /$, and $/ \mathrm{w} /$. The speech identification score drastically improved during the training. The improvement in non-native wordinitial rl distinction was clearly associated with the changes in the perceptual, articulatory, and neuronal spaces, which represent dissimilarities between the non-native and native phonemes in each domain assessed through perceptual, palatographic, and neuromagnetic measurements. Significant difficulty in identification of consonant-rl clusters, however, remained for some trainees even after one year of training. Analyses of palato-lingual contact patterns during word articulation suggested that more articulatory errors tend to occur in consonant-rl clusters than in other phonotactic contexts in such a way that both $/ 1 /$ and $/ \mathrm{r} /$ are substituted with Japanese /r/ sometimes associated with a vowel inserted. Results suggested that the L2 phonological system can be acquired through a multimodal training, although it is strongly interfered with by L1 phonotactic constraints.

2pSCb6. Patterns of vowel-to-vowel coarticulation in Greek and English. Areti Okalidou (Dept. of Logotherapy, Tech. Educational Inst. of Patras, 26334 Koukouli, Patra, Greece) and Laura L. Koenig (Haskins Labs., New Haven, CT 06511)

Many previous studies have indicated that coarticulatory behavior differs systematically across languages. One specific hypothesis, made by Manuel and Krakow (1984), is that languages with larger vowel inventories show reduced vowel-to-vowel coarticulation because their crowded vowel spaces impose more stringent requirements for maintaining phonological distinctiveness. The present study is designed (a) to test this prediction in Greek versus English, which have small and large vowel inventories, respectively; and (b) to investigate which parameters of the vowel system are most responsible for shaping language-specific coarticulatory constraints. Adult monolingual speakers of each language were recorded, producing VCV utterances embedded in phonologically similar carrier phrases. Vowels included all five vowels of Greek, /i,e,a,o,u/, and their closest counterparts in American English, and the medial consonant varied between labial and alveolar places of articulation. To provide an independent measure of production variability for each vowel, speakers also produced the vowels in real words of the form $/ \mathrm{pVt} /$ or $/ \mathrm{bVt} /$, embedded in the same carrier phrases. Preliminary data are presented for two speakers of each language, and discussed in terms of vowel type, distribution within the vowel space, and the size of vowel areas within $F 1-F 2$ space. 
2pSCb7. Production of Japanese durational contrasts by native and nonnative speakers. Yukari Hirata (Univ. of Chicago, Dept. of Linguist., 1010 E. 59th St., Classics 304, Chicago, IL 60637, yh16@midway.uchicago.edu)

Non-native speakers' production of Japanese durational contrasts, and the development of their abilities, are examined in relation to native speakers' production. Two native speakers and two beginning learners produced 2-4 mora words contrasting in either consonant or vowel length. The learners also produced the same set of words after 4 weeks of perceptual training [Y. Hirata, CLS34 Phonetics Panel (forthcoming)]. Acoustic analyses of the native speaker productions indicate durational systematicity not only in the consonant pairs, as in M. Han [Phonetica 49, 102-127 (1992)], but also in the vowel pairs: the average ratio of short to long vowels was 1:1.8-2.0 regardless of context and speaker. Furthermore, a linear relationship was found between the number of morae and word duration, extending Port et al. [J. Acoust. Soc. Am. 81, 1574-1585 (1987)]. The learners' results before training indicate an inability to produce the durational contrasts, e.g., subject A's average durational ratio for the vowel pairs was 1:1.2. After training, the ratio became closer to that of the native speakers, viz., 1:1.6-1.7, while subject B's did not show a linguistically significant change. Subject A's results contrast even with those of Han's advanced subjects, and illuminate the upper limit for L2 learners with training.

2pSCb8. Acoustic-phonetic analysis of the acquisition of American English tense-lax contrasts by native speakers of Chinese. Catherine L. Rogers (Dept. of Speech and Hearing Sci., Ohio State Univ., 110 Pressey Hall, 1070 Carmack Rd., Columbus, OH 43210, rogers.140@osu.edu)

In a study of the perception of Chinese-accented English by native American English-speaking listeners [C. Rogers and J. Dalby, J. Acoust. Soc. Am. 100, 2725(A) (1996)], in which performance on seven segmental error category variables was correlated with intelligibility at the sentence level, vowel tenseness was found to be most strongly correlated with connected-speech intelligibility $(r=0.76, p<0.001)$. Analyses of these results across the eight Chinese speakers showed that the average performance of the four most intelligible speakers (measured in terms of sentence percent-correct performance) on words targeting tense-lax contrasts surpassed that of the four least intelligible speakers by more than $30 \%$. Analyses of both durational and spectral properties of the target lax phonemes /I, $\varepsilon$, and U/ produced by the Chinese speakers will (1) explore the acoustic properties responsible for the difference in performance on the target lax phonemes across the two groups of speakers, (2) compare results across the three target phonemes, and (3) investigate the hypothesis that acquisition of the tense-lax contrasts is associated with more nativelike implementation of phenomena realized only at the connected-speech level (e.g., phrase-final lengthening). [Work supported by NIDCD.]

2pSCb9. Perceived vowel quantity in Swedish: Native and British listeners. Dawn M. Behne (Norwegian Univ. of Sci. and Technol., N-7043 Trondheim, Norway), Kirk P. H. Sullivan, and Peter E. Czigler (Ume Univ., SE-901 87 Ume, Sweden)

In many languages, vowels are characterized by their use of contrastive phonological vowel quantity and vowel quality. In Swedish, vowels have traditionally been described as being distinct in quality as well as having a phonological distinction between short and long vowel quantities. In English, however, phonological distinctions among vowels are described as primarily qualitative. This investigation examines the perceptual use of vowel duration and the first two vowel formant frequencies in distinguishing Swedish vowel pairs by three groups of listeners: native Swedish listeners (SS), British English listeners who do not know Swedish (EE), and British listeners who know Swedish well (ES). For each of three pairs of Swedish vowels (IPAKiel[ $[=$ DC]IPAKiel-[I], IPAKiel[ $[0=D C]-$ IPAKiel[O], IPAKiel[A=DC]IPAKiel-[a]), /kVt/ words were resynthesized having ten degrees of vowel duration and ten degrees of $F 1$ and $F 2$ adjustment. Listeners' responses and reaction times in a rhyming task show that unlike native listeners, L2 (i.e., ES) listeners distinguish Swedish vowel quantity using duration almost exclusively, which is consistent with what they have been taught, whereas TimesEE listeners respond as expected for a comparable English vowel continuum, using both vowel duration and spectra. These results are discussed in terms of the different stategies used by the different listeners.

2pSCb10. Dynamic information in French vowel identification by native and non-native listeners. Terry $L$. Gottfried and Jonathan S. Neville (Dept. of Psych., Lawrence Univ., Appleton, WI 54912)

French /t/-vowel and /t/-vowel-/t/ syllables were spoken by a native speaker in a constant sentence frame. Target syllables were edited so that native French and non-native listeners heard intact syllables, only the initial and final portion of syllables (silent-center), only initial (initial-only), or only center portions (center-only). Research in American English [e.g., W. Strange, J. Acoust. Soc. Am. 85, 2135-2153 (1989)] has demonstrated that listeners accurately identify vowels in silent-center consonant-vowelconsonant syllables; identification of initial- or final-only portions is very poor. However, French syllables are more commonly consonant-vowel in structure; for this reason, native listeners might identify initial-only portions nearly as accurately as silent-centers. By contrast, Americans whose second language is French might perform more poorly on silent-center French syllables than on intact syllables, and most poorly on initial-only. Findings suggest that indeed native French listeners identify initial-only syllables as accurately as silent-center and intact syllables, except for confusions of oral-nasal contrasts (e.g., /a/ vs $/ \varepsilon /$ ). Native listeners perform significantly better on TV syllables than TVT syllables, especially in silent-center and initial-only conditions. The pattern of errors for nonnative listeners is different, particularly with respect to less familiar vowel contrasts (e.g., /u/ vs /y/). [Work supported by Lawrence University Faculty Grant.]

2pSCb11. Training American listeners to perceive Mandarin tones: Transfer to production. Yue Wang, Michelle M. Spence, Allard Jongman, and Joan A. Sereno (Cornell Phonet. Lab., Cornell Univ., Ithaca, NY 14853, yw36@cornell.edu)

Training American listeners to perceive the four Mandarin lexical tones has been shown to be effective [Y. Wang, A. Jongman, and J. A. Sereno, J. Acoust. Soc. Am. 103, 3090(A) (1998)]. Eight trainees participated in a two-week training program, after which their tone identification accuracy showed an overall $21 \%$ increase from the pretest to the posttest, and the improvement was generalized to new stimuli and new talkers. Further studies have now been carried out to investigate whether the tone contrasts gained perceptually can be transferred to the production domain without additional training. Before their perception pretest and after their posttest, the same trainees were recorded, producing a list of 80 Mandarin words bearing the four tones. Their productions were judged by 40 native Mandarin listeners in an identification task. Trainees' posttest tone productions were on average $16 \%$ more accurately identified than their pretest productions, indicating significant tone production improvement after perceptual training. Acoustic analyses of the pre- and posttraining productions further reveal the nature of the improvement. Results are discussed in terms of the relationship between non-native tone perception and production as well as learning at the suprasegmental level.

2pSCb12. Point vowels in Japanese mothers' speech to infants and adults. Jean E. Andruski (Audiol. and Speech-Lang. Pathol., Wayne State Univ., 581 Manoogian Hall, 906 W. Warren Ave., Detroit, MI 48202), Patricia K. Kuhl (Univ. of Washington, Seattle, WA 98195), and Akiko Hayashi (Tokyo Gakugei Univ., Koganei, Tokyo 184, Japan)

American, Russian, and Swedish mothers produce acoustically more extreme point vowels $(/ \mathrm{i} /, / \mathrm{u} /$, and $/ \mathrm{a} /)$ when speaking to their infants than when speaking to another adult [Kuhl et al., Science 277, 684-686]. This study examines the three point vowels in Japanese mothers' speech, and 
compares the acoustic structure of infant-directed (ID) and adult-directed (AD) tokens. Three target words containing /i/, /u/, and /a/ (bi:zu, batto, bu:tsu = beads, bat, boots) were recorded while mothers conversed with another native-speaking adult, and with their infants, aged either $5 \frac{1}{2}$ or $8 \frac{1}{2}$ months. $F 1, F 2$, and $F 0$ were measured at vowel onset, center, and offset. Acoustic, results will be compared for AD and ID speech, and expansion of the vowel space in Japanese mothers' speech will be examined. [Work supported by NIH HD35465-01S1.]

2pSCb13. Detection of foreign accent by $L 2$ English listeners. Kimiko Tsukada (SHLRC, School of ELM, Macquarie Univ., NSW 2109, Australia)

This study investigates the question of whether non-native listeners are capable of differentiating native from non-native English talkers when presented with a specific foreign accent. Seven Japanese listeners (six female, one male), five of whom have lived in English-speaking countries for a minimum of nine years, were asked to identify the native talker in a forced-choice paired comparison task. The stimuli used were monosyllabic English words (in $/ \mathrm{CVt} /$ and $/ \mathrm{CVd} /$ ) produced by Australian and Japanese talkers in which vowels were matched on their $F 1, F 2$ at the temporal midpoint and duration. Non-native listeners performed the task more poorly than the native listeners $(60 \%$ vs $79 \%)$. It was observed that some adult non-native listeners have difficulty in recognizing even their own L2 speech production [H. Goto, Neuropsychologia 9, 317-323 (1971)]. Perception errors were spread quite evenly across 11 vowel types. However, vowels such as $/ \varepsilon, / æ /, / \Lambda /$, and $/ /$ induced more errors. This may be related to the acoustic vowel space of their $L 1$ in which, unlike English, the only low vowel /a/ is not cramped by the neighboring vowels. The results revealed large interlistener variability which suggests a complex nature of $L 2$ perception.

2pSCb14. Neurophysiologic correlates of cross-language perception of phonetic categories. Anu Sharma and Michael Dorman (Dept. of Speech and Hearing Sci., Arizona State Univ., Tempe, AZ 85257-0102, anu.sharma@asu.edu)

This study examined neurophysiologic correlates of categorical perception of native and non-native phonetic categories. Behavioral and electrophysiologic responses were obtained in Hindi and English listeners, in response to a stimulus continuum of naturally produced, bilabial $\mathrm{CV}$ stimuli that differed in VOT from -90 to $0 \mathrm{~ms}$. These speech sounds constitute phonenically relevant categories in Hindi but not in English. As expected, the native Hindi listeners identified the stimuli as belonging to two distinct phonetic categories (/ba/ and $/ \mathrm{pa} /$ ) and their discrimination of a stimulus pair across categories was more accurate than their discrimination of a stimulus pair (of equal VOT change) within the /pa/ category. English listeners discrimination accuracy of within- and across-category pairs was at chance. N100 and MMN cortical-evoked potentials which are considered neural indices of preattentive sensory encoding and discrimination were measured. A double-peaked N100 was elicited in English and Hindi listeners. The first peak was elicited by syllable onset, while the latency of the second peak reflected the VOT lead time. Hindi listeners exhibited robust MMNs for the within- and across-category pairs, while English speakers did not, suggesting that the central sensory representation of certain phonetic categories is at least partially altered by linguistic experience.
2pSCb15. Perceptual training with native Japanese speakers on various L2 sounds. Jesica C. Pruitt (Speech and Hearing Sci., Univ. of Washington, 1417 N. E. 42nd St., Seattle, WA 98105, jcpruitt@u.washington.edu), Reiko Akahane-Yamada (ATR-HIP Res. Labs, Soraku-gun, Kyoto, 619-0288 Japan), Winifred Strange (CUNY Grad. School, New York, NY 10036), Rieko Kubo, and Tomoko Takada (ATR-HIP Res. Labs, Soraku-gun, Kyoto, 619-0288 Japan)

It has been found that adults can more robustly acquire segments in a second language (L2) with identification training which uses highly varied training stimuli, i.e., natural tokens from multiple talkers in multiple phonetic contexts. This approach has been applied systematically in crosslanguage training studies in which Japanese speakers were trained to acquire AE /r/ and /l/ [Lively et al., J. Acoust. Soc. Am. 96, 2076-2087 (1994)] as well as AE/s, æ, $\Lambda$ [ [Strange and Akahane-Yamada, J. Acoust. Soc. Am. 102, 3137(A) (1997)]. The current set of experiments expanded this line of research by testing whether the same procedure would be effective in training other consonants $(/ \mathrm{b} /-/ \mathrm{v} /, / \mathrm{s} /-/ \theta /$ and $/ \mathrm{z} /-/ \mathrm{\delta} /)$ and vowels (11 AE vowels). With both consonants and vowels, the previous results were replicated; subjects showed significant improvement from pretest to posttest and showed generalization to an untrained talker. In the vowel training study, transfer of training across speaking style was further examined. Subjects were trained to distinguish vowels in CVC syllables in a carrier sentence context, and were then tested in both sentence and citation contexts. Interestingly, the improvements were significant for sentence contexts but not for citation. Taken together, these studies further our understanding of the complexities of L2 learning and training.

2pSCb16. Recognition of Portuguese vowels by Galician-speaking and Spanish-speaking listeners. Sergio Feijóo, Santiago Fernández, and Ramón Balsa (Departamento de Física Aplicada, Universidad de Santiago de Compostela, 15706 Santiago, Spain, fasergio@usc.es)

Galician, Portuguese, and Spanish are three romanic languages spoken in the Iberian peninsula. Galician and Portuguese are historically related and keep the same vocalic system of common latin: a, $\varepsilon$, e, i, o, o, u. In Spanish the number of vowels was reduced to five: a, e, i, o, u. Nevertheless, Spanish has exerted considerable influence on Galician, contributing to weaken the vocalic contrasts $\varepsilon-\mathrm{e}$ and $\mathrm{o}-\mathrm{o}$. The purpose of these experiments was to assess how Galician- and Spanish-speaking listeners perceived the vocalic system of Portuguese. Stimuli were $\mathrm{pV}$ syllables pronounced in a carrier phrase, where $\mathrm{V}$ was one of the seven vowels. Results show that Galician-speaking listeners have some difficulty in perceiving the contrasts $\varepsilon$-e and $0-0$, probably due to the historical influence of Spanish, and despite the fact that $F 1$ and $F 2$ of the Portuguese vowels were similar to those reported for Galician vowels. Spanish-speaking listeners identified $\varepsilon-\mathrm{e}$ as a single vowel e, and $\mathrm{o}-\mathrm{o}$ as a single vowel o, associating the $\mathrm{L} 2$ vocalic inventory to their own vocalic system. Discussion is based on the acoustic characteristics of vowels in those languages.

2pSCb17. Aerodynamic constraints on sound change: The case of syllabic sibilants. Alan C. L. Yu (1203 Dwinelle Hall, Dept. of Linguist., Univ. of California, Berkeley, CA 94720-2650, aclyu@socrates.berkeley.edu)

High vowels are known to assimilate in place of articulation and frication to a preceding sibilant. Such an assimilation process is found in an historical sound change from Middle Chinese to Modern Mandarin (e.g., */si/ became [sz] "poetry"). However, such assibilation is systematically absent when the vowel is followed by a nasal consonant. This paper investigates the co-occurrence restriction between nasalization and frication, demonstrating that pharyngeal pressure is so significantly vented during the opening of the velic valve that the necessary pressure buildup behind the constriction of a fricative is consequently severely diminished, resulting in no audible turbulence. It reports the aerodynamic effects of nasalization on vowels, as spoken by a native speaker of American English (presumed to parallel the phonetic conditions present in Middle Chinese). The results reveal that in comparison to oral vowels the pharyngeal pres- 
sure, volume velocity, and particle velocity decrease dramatically when high vowels are nasalized. Based on this study, a physical motivation for the phonological patterns with respect to the interaction between syllabic sibilants and nasal is advanced. [This work is supported by the President's Undergraduate Fellowship 1997-1998.]

2pSCb18. Does interspeaker variation in habitual speaking rate predict the size of the acoustic vowel space? Ying-Chiao Tsao (Dept. of Special Ed./Communicative Disord., Univ. of Nebraska, Kearney, NE 68849, tsaoy@platte.unk.edu) and Gary Weismer (Univ. of Wisconsin, Madison, WI 53706)

In a previous study [Tsao and Weismer, J. Speech Lang. Hear. Res. (1997)], it was demonstrated that interspeaker variation in habitual speaking rate is predictive of interspeaker variation in maximal speaking rate. Thus speakers who are habitually slow speakers do not seem to be able to produce as fast a maximum rate as speakers who are habitually fast. This finding was interpreted to mean that some component of interspeaker variation in habitual speaking rate reflects neurological predispositions, rather than (for example) voluntary "choices" of different rates. The present study explores the hypothesis that habitually slow and fast speakers may have differently sized acoustic vowel spaces. The hypothesis is derived from previous observations that within speakers, the size of the acoustic vowel space fluctuates with speaking rate. Results suggest that there is substantial overlap between the vowel spaces of habitually slow and fast speakers, but that the slow speakers do tend to have larger vowel spaces. [Work supported by NIH.]

2pSCb19. Russian vowels in mothers' speech to infants and adults. Jean E. Andruski (Audiol. \& Speech-Lang. Pathol., Wayne State Univ., 581 Manoogian Hall, 906 W. Warren Ave., Detroit, MI 48202), Patricia K. Kuhl (Univ. of Washington, Seattle, WA 98195), L. A. Chistovich, E. V. Kozhevnikova, V. L. Ryskina, and E. I. Stolyarova (Early Intervention Inst., St. Petersburg, Russia)

Cross-linguistically, when mothers address their infants they produce acoustically more extreme point vowels $(/ \mathrm{i} /, / \mathrm{u} /$, and /a/) than they produce when speaking to another adult [Kuhl et al., Science 277, 684-686 (1997)]. This study examines three nonpoint vowels in Russian (/e/, /o/, /'i/) and compares their acoustics in infant-directed (ID) and adult-directed (AD) speech with the acoustics of Russian point vowels in AD and ID speech. Six target words containing the nonpoint vowels in stressed syllables were recorded while ten mothers conversed with their infant and another adult. $F 1, F 2$, and $F 0$ were measured at vowel onset, center, and offset. As with the point vowels, the acoustic structure of all three vowels differs significantly in ID and AD speech. F2 tends to move to an acoustically more extreme position in each case. In addition, nonpoint ID vowels show more vowel-inherent formant movement across the course of the vowel. Formant movement may provide an important cue to vowel identity, and its exaggeration in ID speech may increase the likelihood that infants incorporate formant movement into their mental representation of a vowel. Significant differences in formant structure were found for vowels in one-, two-, and three-syllable words, including vowels with short mean durations.

2pSCb20. Identifying novel samples of spoken Korean. Z. S. Bond and Verna Stockmal (Dept. of Linguist., Ohio Univ., Athens OH 45701, zbond1@ohiou.edu)

Listeners can identify familiar foreign languages without understanding them on the basis of their sound or acoustic signature. Listeners need only brief exposure to a novel language to learn to identify it from its sound pattern [Bond et al., Lang. Sci. 20, 353-367 (1998)]. The current experiments replicated the previous study using a different target language. In the first experiment, listeners distinguished Korean from languages which employ syllable rhythm. In the second experiment, listeners separated Korean from other East Asian languages. In both experiments, listeners made judgments either without exposure (control) or after exposure to $10 \mathrm{~min}$ of spoken Korean. Listener responses were converted to $A$, a nonparametric measure of discriminability. In experiment 1 , listeners in the control condition responded at chance, mean $A=0.47$. After exposure, listeners identified Korean above chance levels, mean $A=0.71$. Listeners tended to confuse Korean with Asian languages rather than with African or European languages, suggesting that voice and speech style influenced their judgments. In experiment 2, the control group had extreme difficulty identifying Korean, mean $A=0.28$. Brief exposure enabled listeners to develop a representation of the sound of Korean in that they produced more hits and fewer misses to the target (mean $A=0.71$ ).

2pSCb21. Acoustic studies comparing Danish vowels, British English vowels, and Danish-accented British English vowels. Anja Steinlen and Ocke-Schwen Bohn (English Dept., Aarhus Univ., DK-8000 Aarhus C, Denmark, engas@hum.aau.dk)

Few studies have examined the production of English vowels by native speakers of languages with vowel inventories as large as, or larger than, English. Danish is such a language, whose vowels are unevenly distributed in the vowel space, with a densely populated upper portion and a sparsely populated lower portion of the vowel space. This paper reports on acoustic comparisons of British English vowels as produced by ten native speakers of British English and of Danish, and of Danish vowels as produced by ten native speakers of Danish. Danish and English vowels were produced in CVC syllables in a variety of consonantal contexts. Acoustic analyses revealed temporal, spectral, and dynamic differences between the vowels produced by the speaker groups. The results of this study suggest that comparisons of vowels across languages, as well as analyses of the productions of non-native speakers, are incomplete and may even be misleading unless the effects of consonantal context are taken into account. The results of this study also provide an interesting test case for Flege's speech learning model, which makes predictions concerning the learnability of foreign language vowels based on their degree of difference from native language categories. 


\title{
Session 2pSPa
}

\section{Signal Processing in Acoustics and Engineering Acoustics: Smart Microphones and Audio for Teleconferencing}

\author{
Gary W. Elko, Cochair \\ Acoustics and Speech Research, Bell Laboratories, Lucent Technologies, 500 Mountain Avenue, Murray Hill, \\ New Jersey 07974, USA \\ Jens Blauert, Cochair \\ Communication Acoustics, Ruhr Universität Bochum, D-44780 Bochum, Germany
}

Chair's Introduction-1:55

Invited Papers

2:00

2pSPa1. Steerable adaptive first- and second-order differential microphones. Gary W. Elko (Acoust. and Speech Res., Bell Labs, Lucent Technologies, 500 Mountain Ave., Murray Hill, NJ 07974, gwe@ research.bell-labs.com)

Directional microphone arrays are spatial filters that can be designed to improve the sound quality of speech in audio telecommunication by removing acoustic signals arriving from undesired directions. Recently, there has been a large interest in adaptive microphone systems (also sometimes referred to as "smart microphones"), since these systems automatically self-adjust their spatial response to minimize acoustic interference and thereby increase the desired signal-to-noise ratio. The talk will present adaptive firstand second-order differential microphone arrays that have fixed look directions dependent on the array geometry. A generalized version of the adaptive first-order system will then be presented where the look direction can be arbitrarily oriented (independent of the array spatial orientation). The commensurate problem of acoustic echo cancellation with adaptive microphones will also be discussed and some potential solutions given.

2pSPa2. Integrating acoustic echo cancellation with adaptive beamforming microphone arrays. Walter Kellermann (Lehrstuhl f. Nachrichtentechnik I, Univ. at Erlangen, Nurnberg, Germany, walter.kellermann@e-technik.fh-regensburg.de)

In hands-free and untethered man-machine speed communication, acoustic echo control (AEC) is widely acknowledged as a critical issue determining, e.g., the quality of a full-duplex dialogue, or the speech recognition performance of voice control applications. In comparison to single microphones, microphone arrays (MAs) allow for an improved acoustic front-end by increased spatial selectivity and the flexibility to track moving sources. It is argued that in most cases, however, this will not suffice to eliminate the need for AEC. The most effective solution to AEC is provided by echo cancellation systems, which model the acoustic echo path from the source to the receiver by an adaptive digital filter. While the echo path for a time-invariant MA can be modeled by a single adaptive filter, adaptive beamforming MAs require a time-variant filter capable of tracking the change of the beamforming characteristic instantaneously or, alternatively, one echo canceller for each sensor of the array. Given the considerable computational load for acoustic echo cancelers, a concept is proposed here that limits the beamforming to several fixed beams and foresees one echo canceler for each beam. In addition to computational efficiency, this system provides synergies enhancing the control of the acoustic echo canceler as well as source finding for beamforming.

\section{2:40}

2pSPa3. Self-calibrating microphone array. Jens Meyer (Dept. of Electroacoustics, Darmstadt Univ. of Technol., Merckstr. 25, 64283 Darmstadt, Germany, J.Meyer@uet.tu-darmstadt.de)

A popular application for microphone arrays is speech pick up in situations where a close spacing between talker and microphone is not suitable. A beamformer is used to achieve a directivity pattern that maximizes the ratio between the desired signal and noise sources. Here a fixed beamformer is evaluated. A fixed beamformer means that the coefficients are set at one time and then remain constant. Many design methods are known for fixed beamformers. Most of these methods assume ideal microphone array characteristics like precise sensor positions, perfect phase and gain of the sensors, and free-field conditions of the acoustical field. In many practical applications these conditions are not fulfilled. The disregard of the free-field condition in some speech pick-up applications (e.g., array on the top of a monitor, array as a hearing aid) especially can cause severe distortions of the desired beam pattern. This presentation suggests an approach that includes the nonideal effects in the design process of the beamformer. The directivity pattern is therefore measured at some discrete angles. The measured data are then compared with a predetermined directivity pattern and the beamformer coefficients are optimized such that this pattern is approximated as closely as possible. 

andrew.rimell@bt.com)

Developments in communications technology, such as audio-visual conferencing and telepresence, have created a new set of demands for audio delivery systems. In the past, a telephone handset, with its limited transducers, was adequate for simple spoken communication; however, future multiuser communication systems and immersive machine interfaces will be greatly enhanced by using high-quality three-dimensional audio. Such three-dimensional sound systems can position individual talkers' voices in space around the user, creating a greater feeling of immersion and improving intelligibility. This presentation discusses the design and implementation of a number of spatial audio system implementations. The applications range from a SmartSpace chair (a single-user environment using a transaural system) through to a VisionDome (a large multiuser environment using a combination of ambisonics and nonlinear panning). Theoretical design and practical implementation issues are discussed, including problems encountered and how they were overcome.

2pSPa5. Audio teleconferencing systems with sound localization effects. Shigeaki Aoki and Manabu Okamoto (Nippon Telegraph and Telephone Corp., Human Interface Labs. 1-1, Hikarinooka Yokosuka, Kanagawa 239-0847, Japan, aoki@nttspch.hil.ntt.co.jp)

In teleconferencing, conversation should flow naturally between separate sites. The acoustic design should, of course, be able to at least convey the correct volume. However, sound localization is also very important for realizing the kind of virtual reality world that is teleconferencing. Coordinating the visual image (a speaker's face) and the sound image (his or her voice) creates a kind of virtual environment in which the participants at each site feel as if they are having a conversation in the same room. Two prototype teleconferencing systems with sound localization effects have been developed. The more primitive of the two is a small teleconferencing system that can connect two sites with stereo reproduction in a way that utilizes psychological acoustic phenomena. The other is a high-presence system that aims at achieving a high level of ambience among three separate sites. Its features and key technologies are natural images on a large super-high-resolution display, good sound localization from remote locations through the use of a multichannel sound image localization system, and high-definition images as a result of HDTV coding and transmission. The proposed multichannel sound system comprises four subsystems, one each for sound input, reproduction, acoustic feedback control, and transmission.

2pSPa6. Robust and adaptive spatialized audio for desktop conferencing. Darren B. Ward (School of Elec. Eng., Univ. College, UNSW, Canberra ACT 2600, Australia, d-ward@ee.adfa.oz.au) and Gary W. Elko (Bell Labs, Lucent Technologies, Murray Hill, NJ 07974)

For multi-talker teleconferencing, providing multichannel audio to the listener has been advocated as an alternative to a conventional single audio channel. The use of multichannel audio provides a means of supplying the listener with spatialized audio cues. Specifically, it permits binaural signals to be delivered to the listener, which has distinct advantages for multi-talker teleconferencing. First it allows remote talkers to be virtually placed around the listener, providing the listener with the ability to easily discriminate between talkers based on their virtual location. Second, if a binaural microphone is used to acquire the talker's speech signal, the listener can receive this binaural signal directly. Binaural signals can be delivered to a listener through headphones or using a loudspeaker array (which is more preferable for video conferencing). However, the use of loudspeaker arrays requires the listener's head to be accurately positioned or else the spatialized audio effect will be lost. This paper contains discussion on the means of delivering binaural signals using loudspeaker arrays that allow the listener to move her or his head without losing the spatialized audio effect.

\section{4:00-4:20 Break}

4:20

2pSPa7. Chat space models. Michael Cohen (Spatial Media Group, Univ. of Aizu 965-8580, Japan, mcohen@u-aizu.ac.jp)

Shared virtual environments, especially those supporting spatial sound, require generalized control of user-dependent media streams. Nonimmersive perspectives in virtual environments enable fluid paradigms of perception, especially in the context of frames-of-reference for conferencing and musical audition. Traditional mixing idioms for enabling and disabling various sources employ mute and solo functions, which, along with cue, selectively disable or focus on respective channels. Exocentric interfaces which explicitly model not only sources, but also location, orientation, directivity, and multiplicity of sinks, motivate the generalization of mute/solo \& cue to exclude and include, manifested for sinks as deafen/confide \& harken, a narrowing of stimuli by explicitly blocking out and/or concentrating on selected entities. Such functions can be applied not only to other users' sinks for privacy, but also to one's own sinks for selective presence. Figurative representations of these functions are introduced, virtual hands to be clapped over avatars' ears and mouths. An audibility protocol is described, comprising revoke, renounce, grant, and claim methods, invocable by these narrowcasting commands to control superposition of soundscapes, and a taxonomy of modal narrowcasting functions is proposed. Applications include groupware for collaboration and teaching, teleconferencing and chat spaces, and authoring and manipulation of distributed virtual environments. 
2pSPa8. Spatial voice activity detection using a microphone array noise canceller. Michael W. Hoffman (Univ. of Nebraska, 209N WSEC, Lincoln, NE 68588-0511) and Zhao Li (Motorola, Inc., Austin, TX 78758)

An array of microphones is used for both noise suppression and spatial voice activity detection (SVAD). A generalized sidelobe canceller (GSC) is employed for adaptive interference rejection and the signals in the noise canceller are also exploited to provide desired signal activity detection. The SVAD shows promising results for environments with moderate reverberation and single or multiple interfering sources. For direct-to-reverberant energy ratios of 4 to $11 \mathrm{~dB}$ and signal-to-interference power ratios of -10 to 10 $\mathrm{dB}$, speech detection miss rates are generally below 5\%, while false alarm rates range from $10 \%$ to $40 \%$. Most scenarios generally resulted in the false alarm rates between $10 \%$ and $20 \%$. A key advantage of the GSC-based SVAD is its computational simplicity. In addition, the proposed technique is quite suitable for multiple interfering sources as well as nonstationary interference such as competing speakers. The system has been tested with a standard CELP speech coder.

5:00

2pSPa9. Speech reconstruction from a noisy reception signal. Michiko Kazama (Acoust. Consultant, Tokyo, Japan), Mikio Tohyama (Kogakuin Univ., Tokyo, 192-0015 Japan), and Akira Morita (NHK Sci. and Tech. Res. Labs., Tokyo, 157 Japan)

Noise reduction is a fundamental issue of smart microphone systems or a hearing aid. Noise reduction by spectral subtraction has been investigated for speech signals. However, identifying whether frame is a speech or a silence portion is difficult under nonstationary noisy conditions when using this method. Extracting the desired speech based on the sinusoidal wave model [T. Quatieri and R. Mcaulay, IEEE ASSP 34, 1449-1464 (1986)] was investigated. It was confirmed that intelligible speech sound could be synthesized using only five dominant sinusoidal waves [M. Kazama et al., 5th ICSV 2079-2086 (1997)]. In this article, a new noise reduction method by extracting the dominant sinusoidal waves in each frame $(32 \mathrm{~ms})$ according to the energy ratio of the signal to noise was proposed. The signal-to-noise ratio was improved by $10 \mathrm{~dB}(\mathrm{~S} / \mathrm{N}$ ratio) when the original $\mathrm{S} / \mathrm{N}$ ratio was $0 \mathrm{~dB}$. Speech quality could also be improved by reconstructing the higher harmonics from the noisy vowels using the frame-dependent comb filters. This reconstructed speech sound will be demonstrated at the presentation. This method might implement a fundamental technology of the smart microphone systems, including hearing aids. [Work supported by Housou Bunka Fund.]

\section{Contributed Papers}

\section{$5: 20$}

2pSPa10. Improvements in teleconferencing sound quality and gain before feedback through the use of DML technology. Peter Mapp (Peter Mapp Assoc., Colchester CO3 4JZ, UK) and Henry Azima (New Transducers, Stonehill, Huntingdon, UK)

One of the primary limitations of teleconferencing systems is the premature onset of acoustic feedback. The current trend toward the use of desktop personal computers as the teleconferencing platform, rather than dedicated and acoustically treated video conferencing rooms, further exacerbates the problem. Local, sound reflecting surfaces including the desktop, conference table, or computer screen, etc., can cause undesirable specular reflections from the loudspeakers to occur. These are then picked up by the system microphone(s), causing the formation of strong comb filtering and resultant sound coloration, reduced speech clarity, and reduced gain before feedback. Historically, a number of approaches have been adopted to help overcome such effects, including echo cancelling, phase shifting and "smart" microphone techniques. The paper illustrates the effects of such reflections and shows that distributed mode loudspeakers can produce less boundary interactions and comb-filtering effects. Experimental data are presented to show that DML technology can passively offer a 4-7-dB improved gain before feedback advantage over conventional cone devices in typical teleconferencing applications. The fact that significant gains in feedback margin and reductions in potential coloration are achieved without the need for additional DSP or active techniques implies a radically different and advantageous acoustical radiation mechanism.

\section{5:40}

2pSPa11. A method for echo cancellation in audio signals using the genetic algorithm. Slawomir K. Zielinski (Tech. Univ. of Gdansk, Sound Eng. Dept., ul. Narutowicza 11/12, 80-952 Gdansk, Poland, slawek@ sound.eti.pg.gda.pl)

A new method for echo cancellation is proposed. This method is based on inverse models of systems causing the echo in transmission channels. Parameters of these models are optimized using the genetic algorithm. In general, the inverse models that are used for echo cancellation can be divided into several categories according to their complexity. In the simplest case, the models consist of digital delay lines, scalers, and adders. The more complex models use also a nonlinear filtration, which in the general case may be a nonstationary one. The experiments and informal subjective listening tests showed that the proposed method allows for the perceptually efficient suppression of echo which was caused by multiple reflections providing delayed and scaled copies of the original signal. An additional preprocessing employing a calculation of the correlation function of the input signal can diminish the computational complexity of the proposed algorithm. Some examples of echo cancellation results obtained with the use of the proposed method are presented and illustrated with sound examples.

\section{6:00}

2pSPa12. A third octave real time VXI analyzer. Alberto Martin, Manuel Recuero, Juan Manuel Lopez, and Mariano Ruiz (Instituto Universitario de Investigacion del Automovil, Universidad Politecnica de Madrid, Crta. de Valencia Km.7, 28031 Madrid, Spain, amartin@insia.upm.es)

A digital signal processing system with VXI interface has been developed in a C-size board conforming to VXI 1.4 specification, and is made up of two blocks. The first one implements a message VXI interface and the second the digital processing itself, based in a 49-MHz TMS320C31. The system includes a complete software to customize for a specific application. The VXI interface has been gifted with a real time operating system and a software parser for building a SCPI (standard command for programmable instruments) translator. The software developed for the digital signal processing system has been built in several modules written in $\mathrm{C}$ language and $\mathrm{C} 30$ assembler in order to improve the velocity. The utility of this system is to realize customizable and specific digital signal processing without the necessity of acquiring complex and expensive VXI boards that do not allow modification of the commands, the specific algorithm, or the firmware. In order to show the versatility of the system, a one-third-octave real time analyzer has been developed in agreement with ANSI S1.11. 


\title{
Session 2pSPb
}

\author{
Signal Processing in Acoustics: Signal Processing (Poster Session) \\ John Impagliazzo, Chair \\ Naval Undersea Warfare Center, Code 8212, Building 679, Newport, Rhode Island 02841, USA
}

\section{Contributed Papers}

\begin{abstract}
All posters will be on display in the Poster Gallery from Monday to Wednesday, 15-17 March. Contributors will be at their posters from 2:30 p.m. to 4:30 p.m. on Tuesday, 16 March.
\end{abstract}

2pSPb1. Improving the performance of ultrasonic range meters. Monica Alonso-Sanudo, Cesar Gracia (ETSI Telecomunicacion, 28040 Madrid, Spain), and Pedro Olmos (CIEMAT, Avda Complutense 22, 28040 Madrid, Spain)

Range meters based on the time of flight of ultrasonic pulses are instruments widely used in a large number of daily operations. However, their performance may be seriously degraded when the environment has plenty of spurious obstacles masking the true target, a situation not rarely encountered in practice. Here some methods to reduce the influence of such echoes on monofrequency, wide-aperture range meters are analyzed. The discussion is focused on the use of that information contained in the pulse that allows one to find most of the target features. Relevant parameters to play with are the ratio between transmitted and received pulse lengths and energies, as well as the time evolution of the echo pattern. These techniques, suited for low-cost and small-size equipment, have been simulated with the help of a simple geometric ray model, showing the significant improvement achieved in the rejection of those spurious echoes that would lead to erroneous range determination if only time counting were implemented. In addition, experimental results obtained with a uPbased prototype employing $40-\mathrm{kHz}$ ultrasound pulses and apertures of 30 deg are presented and discussed.

2pSPb2. Hands-free teleconferencing unit using auxiliary loudspeaker and duo-filter echo canceller DSP. Suehiro Shimauchi, Yoichi Haneda, and Yutaka Kaneda (NTT Human Interface Labs., 3-9-11, Midori-cho, Musashino-shi, Tokyo, 180-8585 Japan, sima@splab.hil.ntt.co.jp)

In many telecommunication systems, hands-free operation is becoming popular. For this to be comfortable and easy, high echo cancellation performance and a simple setup are required. This paper presents a new hands-free teleconferencing unit that consists of a main loudspeaker, an auxiliary loudspeaker, a microphone, and an echo canceller DSP board. The auxiliary loudspeaker is used to reduce the acoustic coupling between the main loudspeaker and the microphone by reproducing the anti-phase sound of the main loudspeaker. Since the auxiliary loudspeaker is set very close to the microphone, its sound level is low enough for users not to hear. Experimental results showed a reduction in acoustic coupling of about $6 \mathrm{~dB}$ from $200 \mathrm{~Hz}$ to $1 \mathrm{kHz}$. This unit also incorporates a newly developed acoustic echo canceller (AEC) DSP. The AEC algorithm, which is based on a duo-filter control system [Y. Haneda, S. Makino, J. Kojima, and S. Shimauchi, "Implementation and Evaluation of an Acoustic Echo Canceller Using Duo-Filter Control System," in Proc. EUSIPCO96 (1996), Vol. 2, pp. 1115-1118], helps to produce good and stable performance even during double-talk.
2pSPb3. Approximation of dummy-head recording technique by a multimicrophone arrangement. Nataliya Tohtuyeva and Volker Mellert (Dept. of Phys. and Acoust., Univ. of Oldenburg, D-26111 Oldenburg, Germany)

Head-related recording and reproduction systems rely on the two-ear microphones of a dummy head. However, the dummy head is sometimes difficult to use, e.g., due to size and weight. Additionally, its geometry, which gives the important diffraction pattern information for sound localization, is fixed. A microphone array, which approximates the head-related transfer functions, has fewer restrictions. Theoretical evaluation of a simple microphone array, which takes psychoacoustic-determined frequency weights for phase and magnitude into account, is presented. Results of practical sound localization test with this array are reported for sound sources in the horizontal plane. [Work supported by the German Science Foundation (DFG).]

2pSPb4. On the subspace methods for direction-of-arrival estimation in underwater acoustics. Miloud Frikel and Victor Barroso (IST/ISR, Av. Rovisco Pais 1, Torre Norte, Piso 7, 1096 Lisbon, Portugal)

The problem of bearing estimation appears in a vast number of applications, e.g., radar, sonar, communication systems, ... . In this study, an investigation of the passive angular location of sources in underwater acoustics using an array of sensors is presented. First, eigenstructure/ noneigendecomposition methods for direction-of-arrival (DOA) estimation of narrow-band/wide-band signals' wave fronts are studied. The noneigenanalysis-based techniques are emphasized because of their weak computational load. The studied methods are of high resolution such as (music, minimum-norm, their propagator, ...). The eigenstructure based techniques on the exploitation of two principal properties: first, the data matrix has $(N-P)$ multiplicity of its smallest eigenvalues, with $N$ and $P$ as the number of sensors and sources, respectively, and the eigenvectors corresponding to the minimal eigenvalues; these methods have better performance than the classical methods. A comparative study of these types of subspace methods is presented. Finally, a robust method to improve the accuracy of the estimation of the direction-of-arrival of the sources is developed. The results of simulations are provided to illustrate the theoretical predictions. [This work was partially funded by FEDER and PRAXIS XXI, under contract PRAXIS/3/3.1/TPR/23/94.]

2pSPb5. Blind source separation. Miloud Frikel, Victor Barroso, and Joao Xavier (IST/ISR, Av. Rovisco Pais 1, Torre Norte, Piso 7, P-1096 Lisboa Codex, Portugal, frikel@isr.ist.utl.pt)

In signal processing the received data can be modeled as a superposition of a finite number of elementary source signals and an additive noise. Generally, in a multi-sensor environment application, such as underwater acoustics, the principal objective is estimating the number and directions 
of radiating sources. In the last decade, eigenstructure-based methods yielding resolution have been proposed. However, these methods need the model of sources. In many cases, this model is not available, indeed, there is the appearance of the blind methods. Blind technique refers to lack of knowledge about the mixing system. It assumes very weak assumptions either for signals or mixtures. Blind source separation consists in identifying the sensors/sources transfer matrix and retrieving the source signals without resorting a priori information about the above matrix. It exploits only the information carried by the received signals themselves. The potential benefit of such a blind approach is that source separation is essentially unaffected by errors in the propagation model or in array calibration whereas the classical array processing methods assume that the array geometry is known and the sources are in the far field of the array. This contribution deals with blind source separation using second-order statistics. [This work was partially funded by FEDER and PRAXIS XXI, under Contract No. PRAXIS/3/3.1/TPR/23/94.]

2pSPb6. Envelope filtering of signals in the modulation frequency domain and evaluation of fluctuation strength. Cord Walter and Reinhard Weber (Akustik, Physik, Carl-von-Ossietzky Univ. Oldenburg, 26111 Oldenburg, Germany)

Fluctuation strength assessments with predefined envelope variations are commonly performed with synthesized modulated sounds. The intent of the present study is to change gradually the variability of recorded signals' envelopes for subsequent fluctuation strength evaluations. Manipulation of a signal's envelope was achieved by implementing a filter in the modulation frequency domain. Making use of an algorithm developed by R. Koch [B. Kollmeier and R. Koch, J. Acoust. Soc. Am. 95, 15931602 (1994)], time signals are transformed into (waterfall-like) bandpasstime signals (bandwidth $86 \mathrm{~Hz}$ ) by calculating the spectra of successive frames. From the time-dependent envelopes of the bandpasses, modulation spectra are computed and filtered. Time signals with changed envelopes are resynthesized from the filtered modulation spectra by reversing the former processing stages. The fluctuation strength of the original and modified time signals was computed and compared with the subjective assessments. The results of this comparison are used to validate an algorithm for fluctuation strength calculations of recorded signals.

2pSPb7. Computer-aided systems for prolonged recording and analysis of human bowel sounds. Charalambos A. Dimoulas, George V. Papanikolaou (Dept. of Elec. and Computer Eng., Aristotle Univ. of Thessaloniki, Univ. Campus 540 06, Thessaloniki, Greece), George M. Kalliris, and Costas Pastiadis (Aristotle Univ. of Thessaloniki, Thessaloniki, Greece)

This paper presents the design of noninvasive systems for the investigation of the small bowel motility patterns. The basic idea was to implement dedicated devices for the recording and analysis of human bowel sounds. Properly modified transducers were used for the caption of gastrointestinal sound signals. A data acquisition ambulatory system, consisting of a preamplifier and a recording unit, was initially designed capable for maximum continuous recording of $6 \mathrm{~h}$. After the first experimental results with this system, the necessity for longer recording duration was obvious. For this reason the installation of a computer-based stationary system in a special reformed examination room was the next development step. Triggering of the input data and rejection of the regions with absence of gastrointestinal activity allowed much more recording duration. A computerized signal processing system, which involves equalizing procedures, noise cancellation techniques, and neural network algorithms, was used for the off-line analysis of the recorded data. Novel measures and 2-D and 3-D representation techniques were employed for the exposition of results. The new approach gave flexibility to the system because longer recording times offer more information to the doctor so that the statistical analysis is much more effective and reliable.

TUESDAY AFTERNOON, 16 MARCH 1999

ROOM H104, 2:00 TO 5:40 P.M.

\title{
Session 2pUWa
}

\section{Underwater Acoustics, Acoustical Oceanography, and Physical Acoustics: Acoustics and the Comprehensive Test Ban Treaty II}

\author{
Arthur B. Baggeroer, Cochair \\ Department of Ocean Engineering, Massachusetts Institute of Technology, Cambridge, Massachusetts 02139, USA \\ Martin Lawrence, Cochair \\ Provisional Technical Secretariat, Comprehensive Nuclear-Test-Ban Treaty Organization, Vienna International Centre, \\ A-1400 Vienna, Austria
}

Invited Papers

2:00

2pUWa1. Overview of the infrasound monitoring system for the Comprehensive Nuclear-Test-Ban Treaty. Douglas R. Christie (Provisional Tech. Secretariat, Comprehensive Nuclear-Test-Ban Treaty Organization, Vienna Intl. Ctr., A-1400 Vienna, Austria, dchristie@ctbto.org)

A global network of 60 infrasonic array stations is being established as part of a global monitoring system for verification of the Comprehensive Nuclear-Test-Ban Treaty. These ultrasensitive stations, which are distributed as uniformly as possible over the surface of the globe, will be capable of detecting and locating any nuclear explosion in the atmosphere with yields of $1 \mathrm{Kt}$ or more. In addition to nuclear explosions, this network will detect infrasound from a wide range of man-made and naturally occurring sources, including large chemical explosions, meteors, volcanic explosions, aerodynamic infrasound associated with orographic features, and auroralgenerated infrasound. The propagation of infrasound in the atmosphere is not, as yet, fully understood. The analysis of data from this network should provide considerable insight into the detailed long-range propagation properties of low-frequency sound in a temporally and spatially inhomogeneous medium. 
2pUWa2. Autonomous hydroacoustic stations in support of CTBT-monitoring technology and event screening. Peter C. Wille (FWG, Klausdorfer Weg 2-24, 24148 Kiel, Germany)

No more than six hydroacoustic receiver stations will be monitoring the three world oceans in the framework of the CTBT. Together with five T-wave stations they will synergetically cooperate with the seismic network which alone can neither provide the required accuracy of localization on the southern hemisphere nor the capability of reliable event discrimination of the oceans. To maintain the function of the sparse hydroacoustic network in case of an irretrievable station breakdown, an autonomous auxiliary unit for temporary replacement of fixed cable connected stations has been developed by FWG. The battery-supplied autonmous station, like the permanent stations, suspends the hydrophone at the depth of the SOFAR channel axis. The lightweight construction permits quick deployment and retrieval. The received acoustic signals are processed for prescreening at the station by algorithms with adjustable criteria to discriminate large explosions from natural events of similar energy turnover such as sea quakes and volcanic eruptions. The characteristics of the signals are transmitted by a surface buoy radio link via low orbit satellites to the data center, together with unprocessed, highly suspicious signals for expert analysis.

2:40

2pUWa3. Adaptation of underwater acoustic propagation models to infrasonic atmospheric propagation. Guy V. Norton, Wayne A. Kinney (Acoust. Simulation, Measurements and Tactics Branch, Code 7180, Naval Res. Lab., Stennis Space Center, MS 39529-5004), Dan J. Ramsdale (Neptune Sciences, Inc., Carriere, MS 39426), and Rodney W. Whitaker (Los Alamos Natl. Lab., Los Alamos, NM 87545)

For decades, the U.S. Navy has been engaged in the development of underwater acoustic propagation models to aid in the design and effective use of sonar systems. Much of the expertise created in this development is applicable (directly or by modification) to challenges associated with the use of infrasonic arrays to monitor CTBT compliance. The results of the modification and adaptation of some existing underwater acoustic propagation models to predict and analyze acoustic-gravity wave signals in both a rangeindependent and a range-dependent atmosphere will be reported. Examples are given that include the effects of range-dependent winds on received signal amplitude and arrival direction for the case of an airborne explosive source and a ground-based infrasonic receiver. Frequencies of interest are in the band from 0.02 to $10 \mathrm{~Hz}$, and ranges of interest are $1000 \mathrm{~km}$ or less. [Work sponsored by the U.S. Department of Energy and the Los Alamos National Laboratory.]

3:00

2pUWa4. Naturally occurring sources of infrasound. Alfred J. Bedard, Jr. (Natl. Oceanic and Atmospheric Administration, Environ. Technol. Lab., Mail Code R/E/ET4, 325 Broadway, Boulder, CO 80303)

There are growing numbers of potential applications for infrasonic observing systems. The evolving International Monitoring System for the nuclear test ban treaty (including 60 infrasonic stations) offers exceptional opportunities to make infrasonic data sets widely available and to explore their uses in geophysical studies. This is especially true because of the ability to perform synergistic studies with seismic and hydroacoustic systems in a processing environment where diverse data sets can be readily melded. Natural infrasound sources such as avalanches, earthquakes, geomagnetic activity, meteors, ocean waves, severe weather, turbulence, and volcanoes offer great resources for international cooperative research programs. Sources of low-frequency sound from civilization processes offer a set of parallel opportunities. This paper will review the broad range of investigations possible using both individual and multiple infrasonic observing systems, as well as infrasonic systems in combination with other remote sensing systems.

\section{Contributed Papers}

3:20

2pUWa5. Interpretation of the different infrasounds detected in France during one year in a four-components prototype CTBT station. Elisabeth Blanc, Alexis Le Pichon, and Yves Cansi (CEA-DASE, BP 12, 91680 Bruyeres-Le-Chatel, France, blanc@ldg.bruyeres.cea.fr)

A four-components prototype CTBT station has been installed in France by the Department of Analysis and Monitoring of the Environment of the Atomic Energy Commission, for the development of instrument and methods for infrasound monitoring. The purpose of this paper is to present the characteristics of the main infrasound signals detected during a year. Infrasound from the Concorde supersonic airplane presents different phases corresponding to the different acoustic paths in the different layers of the atmosphere. The number of phases, their apparent velocity, and the signal shape are strongly dependent on the ray trajectory and then on the wind and temperature profiles. Characteristic seasonal variations have been determined. Microbarom signals have been used to follow the evolution of the ocean swell during periods of several days. Severe weather infrasound has been observed, related to lightning activity and convective motions of the atmosphere. Chemical industrial explosions provide interesting well-known sources used for testing the methods and for the development of propagation models. All these events are control events for the permanent evaluation of the detection and location capability of the station. A synthesis of one year's observations is given.
3:40

2pUWa6. Infrasound source location at the prototype international data center. David J. Brown, Charles N. Katz, Ronan Le Bras, Jin Wang, and Anna Gault (SAIC/CMR, Ste. 1450, 1300 N. 17th St., Arlington, VA 22209)

The Prototype International Data Center (PIDC) is developing an operational system to provide temporal and spatial source location information for highly impulsive sources with an equivalent nuclear yield of at least $1 \mathrm{kT}$ in any terrestrial environment. This source information is deduced by a combination of automatic and interactive processing. Detecting the infrasound released by large-scale atmospheric explosions is the most suitable method for the immediate location and characterization of atmospheric sources. The PIDC treats the source location problem uniquely in that a given source can be located using any combination of infrasonic, seismic, or hydroacoustic signals when these signals exist. The highly dynamic aspect of the atmosphere, however, creates problems not traditionally encountered in the seismic or hydroacoustic source location problem. The dynamic nature of these effects will limit the ability to apply detailed propagation models in an automatic operational system, such as is being developed at the PIDC. A seasonal atmospheric model is being used in conjunction with near real-time meteorological data to provide the am- 
bient atmospheric conditions required by the physical propagation model. Results obtained from a ground-truth data set will be presented that demonstrate the operation of the PIDC source location procedure.

\section{4:00-4:20 Break}

\section{4:20}

2pUWa7. Temporal variability in the propagation of infrasonic waves over Hawaii and Alaska. Milton Garces, Kent Lindquist, Roger Hansen (Univ. of Alaska, Fairbanks, AK 99775-7320, milton@hialoha.net), Douglas Drob, and Michael Picone (Naval Res. Lab., Washington, DC 20375)

Temporally varying atmospheric temperature and wind velocity data from ground level up to heights of $250 \mathrm{~km}$ are used to compute travel time curves and azimuth deviations for acoustic waves propagating over the CTBT infrasonic array sites of Hawaii and Alaska. An enhanced formulation of the tau- $p$ method [Garces et al., Geophys. J. Int. (1998)] permits theoretical estimates of travel times and azimuth deviations for infrasonic waves traveling through a stratified, windy atmosphere. Atmospheric data for the season of Winter is obtained from the Naval Research Laboratory MSISE-90 and HWM-93 models, which include tidal and geomagnetic effects. These data will be supplemented with ground-based and satellitebased atmospheric data. An ensemble of circumstances, encompassing infrasonic wave propagation through anomalous environmental conditions as well as studies on site-specific effects, will be presented. Based on the predicted phases, effective detection and source inversion algorithms will be developed at each array.

\section{4:40}

2pUWa8. An optical fiber infrasound sensor. Mark Zumberge and Jonathan Berger (IGPP, Scripps Inst. of Oceanogr., 9500 Gilman Dr., La Jolla, CA 92093-0225, jberger@ucsd.edu)

As part of the International Monitoring System of the CTBT, infrasound signals in the band 0.02 to $4 \mathrm{~Hz}$ must be detected in the presence of ambient noise generated chiefly by wind. The effectiveness of acoustic filters employed in standard infrasound sensors is limited by pressure propagation and attenuation characteristics within the filter. To improve the signal-to-noise ratio, an optical fiber instrument for sensing the integrated pressure variations along a line has been designed. The sensor design consists of optical fibers inside a long, fluid-filled tube, deployed along the ground surface. Integrated pressure variations along the fiber's length are sensed by interferometrically monitoring changes in the optical path length of the fiber. The optical fiber sensor can average over kilometer-scale lengths of arbitrary geometry with an averaging bandwidth governed by the speed of light and thus should offer significant practical advantages in reducing the effect of wind noise, increasing the signal-tonoise ratio over a wide bandwidth.
5:00

2pUWa9. One-year analysis of systematic detection and location of infrasounds in a protoype CTBT station. Alexis Le Pichon and Yves Cansi (CEA-DASE, BP 12, 91680, Bruyères-Le-Châtel, France, lepichon@ldg.bruyeres.cea.fr)

Within the framework of the Comprehensive Test Ban Treaty, the Department of Analysis and Monitoring of the Environment (DASE) of the French Atomic Commission has developed an automatic processing software providing detection of coherent infrasound signals. The infrasound's parameters are calculated by using the progressive multichannel correlation method as a real-time detector. Each week, an automatic bulletin summarizes all detections by giving the wave parameters (velocity and azimuth), the amplitude, the frequency range, and the correlation coefficient. The one-year bulletins enable the identification of most of the infrasounds (sonic boom of supersonic aircraft, mining explosions of known quarries, storms, ocean swell). Most of the man-made infrasounds are produced by quarry blasts and the Concorde. Other infrasounds are produced by severe weather (storms, hail). Excluding false detections, an average number of 40 detections is obtained each day. For some infrasounds, statistics show several phases related to the different reflections in the stratosphere and thermosphere with seasonal characteristics. In order to detect coherent signals with poor signal-to-noise ratio (detection in windy period, fine detection of phases with low amplitudes), the thresholds of detections are set at a low level. In the future, this study will help to determine operational thresholds.

\section{$5: 20$}

2pUWa10. High-resolution measurements of the angle-of-arrival of infrasonic signals in the range 0.5-5 Hz. Ludwik Liszka (Swedish Inst. of Space Phys., Umea Div. Sorfors 634, S-905 88 Umea, Sweden, ludwik@irf.se)

The method used in the present study is a further development of the threshold filtering technique. Signals are transformed using the Morlet wavelet and a sliding window in the domain of the wavelet coefficient magnitude (spectral density). The procedure results in an ampligram, a graph demonstrating the amplitude and phase of components of the signal corresponding to different spectral densities. The ampligram may be further used to determine the number of independent components of the signal using the principal component analysis. The method offers a possibility to detect the statistical properties of signal components: stochastic and deterministic components may be easily distinguished. The most important applications for infrasound measurements are nonlinear filtering of the signal, extraction of weak deterministic components outside the working range of microphone, extraction of different propagation modes, and high-resolution measurements of the angle-of-arrival. The application described here facilitates measurements of the angle-of-arrival for each propagation mode separately.

\section{Contributed Posters}

These posters will be on display from Monday to Wednesday, 15-17 March. Authors will be at their posters from 10:00 a.m. to 12:00 noon on Wednesday, 17 March.

2pUWa11. Surveying infrasonic noise on oceanic islands. Michael A. H. Hedlin, Frank L. VernonIII, Jon Berger, and John A. Orcutt (IGPP, Univ. of California, San Diego, 9500 Gilman Dr., La Jolla, CA 92093-0225, hedlin@ucsd.edu)

An essential step in the establishment of an international monitoring system (IMS) infrasound station is the site survey. The Provisional Technical Secretariat recommends infrasonic noise be recorded with meteorological data at four separate, promising, locations in the vicinity of the nominal station location to identify a secure site which would give the good signal-to-noise while offering easy access to power and telecommunications. A good infrasound station should be sheltered from the prevail- ing winds by gentle topography, trees, and ground cover and located as far as possible from other natural and cultural noise sources. As the IMS network has been designed to give uniform global coverage, some of the stations will be located on oceanic islands. Noise surveys are being conducted at three locations in the Atlantic: Sao Miguel, Azores; Santiago, Cape Verde; and Ascension Island. These island locations are very important as they provide coverage in a region of great monitoring interest. However, they also offer a range of meteorological, topographical, and habitation conditions which result in few suitable locations to deploy an infrasound array. This paper describes preliminary efforts to characterize infrasonic noise levels on these islands and find locations with the necessary practical requirements. 
2pUWa12. On temporal and spatial variations of infrasonic signals at long distances from surface explosions. Sergey N. Kulichkov (Inst. of Atmospheric Phys., Russian Acad. of Sci., Pyzevsky 3, Moscow 109017, Russia)

Temporal and spatial variations of infrasonic signals at the long distances from surface explosions are discussed. The experiments were performed in different seasons during 1989-1991. Surface explosions with yields of 20-70 tons were realized one to two times in a month in runs of four to ten explosions with 10-20-min intervals between them. The receivers were placed both in the zone of acoustic shadow and in the zone of acoustic audibility at the distances about $300 \mathrm{~km}$ from the explosions. Signals from more than 60 explosions were recorded. It was observed that the characteristics of infrasonic signals from similar explosions can vary sufficiently both during one hour and during one day. The variations of those during different seasons were observed too.

TUESDAY AFTERNOON, 16 MARCH 1999

ROOM H2053, 2:00 TO 5:00 P.M.

\title{
Session 2pUWb
}

\section{Underwater Acoustics and Signal Processing in Acoustics: Localization, Estimation and Detection in Underwater Acoustics}

\author{
D. Eric Smith, Chair \\ Applied Research Laboratories, University of Texas at Austin, Austin, Texas 78713-8029, USA
}

Contributed Papers

2:00

2pUWb1. Cell noise at the output of a MFP beamformer in shallow water. Newell O. Booth, Phil W. Schey, and Ahmad T. Abawi (Space and Naval Warfare Systems Ctr., Code D881, San Diego, CA 92152-5000)

Measurements of ambient noise in search cells at the output of linear and adaptive beamformers are presented for data from three arrays deployed simultaneously in the SWellEX-96 experiment (200-m water depth off San Diego, CA). Previous papers by the authors have demonstrated the application of matched-field processing (MFP) to the detection and localization of submerged acoustic sources using the SWellEX-96 arrays. In this paper, the arrays (horizontal, vertical, and tilted-vertical lines) were processed to obtain cell noise spectra in noisy and quiet regions of the range, depth, and azimuth search space. Array noise gain measurements quantitatively compare the noise rejection capability of the arrays and processing methods. Frequency, integration time, array orientation, number of elements, and aperture are important parameters. Results are presented for linear and adaptive beamforming methods using plane-wave, focused plane-wave, and MFP steering vectors. [Work sponsored by the Office of Naval Research.]

\section{$2: 20$}

2pUWb2. Tracking results from the three-dimensional full field processing (FFP) array and the Santa Barbara Channel experiment. Herbert Freese, Yung Lee, Robert Greene, and Peter Mikhalevsky (SAIC, 1710 Goodridge Dr., McLean, VA)

In March of 1998, SAIC under DARPA sponsorship deployed five 170-m, 30-element, vertical line arrays (VLAs) in $200 \mathrm{~m}$ of water in the Santa Barbara Channel. The VLAs were arranged in a pentagon with a 200-m horizontal aperture. Each hydrophone package contained tilt sensors for array localization as well as a thermistor for water temperature measurement. Each VLA also contained four compasses that were used in conjunction with the tilt sensors for passive localization of the array elements. In this presentation the acoustic experiments will be described and a number of results including a comparison of acoustic and nonacoustic localization methods, an environmental characterization, and the results of acoustic measurements using a towed source and surface ships of opportunity will be presented. Specific acoustic results to be presented will include measurements of array signal and noise gain, and the results of tracking the towed source and surface ships using adaptive matched field methods.
2:40

2pUWb3. Comparison of mode-shapes-only source depth estimation with conventional matched field source localization and the impact of array tilt on these processes. Paul Hursky (Lockheed Martin, P.O. Box 180858, Coronado, CA 92178-0858), William S. Hodgkiss, and William A. Kuperman (Scripps Inst. of Oceanogr., San Diego, CA 92093-0701)

Matched field processing (MFP) typically exploits acoustic mode structure using both mode shapes and wave numbers to estimate source location. It is also possible to use mode shapes alone to estimate source depth [E. C. Shang, J. Acoust. Soc. Am. 77, 1413-1418 (1985); T. C. Yang, ibid. 82, 1736-1745 (1987)]. Measurements of multiple frequency towed source transmissions using a vertical line array spanning the lower duct of a summer sound-speed profile (SwellEx 96) were used to compare conventional MFP processing with several variants of mode-shapes-only depth estimation. Sensitivity of these processes to reduced aperture, array tilt, and signal-to-noise ratio was studied in simulated and measured data. Array tilt mismatch was successfully corrected to improve source localization results significantly.

\section{3:00}

2pUWb4. Highly resolved detection in a waveguide with the D.O.R.T. method. Claire Prada, Nicolas Mordant, and Mathias Fink (Lab. Ondes et Acoust., Univ. Paris 7, CNRS, ESPCI, 10 rue Vauquelin, 75005 Paris, France)

The D.O.R.T. method (French acronym for decomposition of the timereversal operator) is a scattering analysis technique that uses an array of transducers in pulse-echo mode. The method was applied to detection and selective focusing on pointlike scatterers through inhomogeneous media [J. Acoust. Soc. Am. 99, 2067-2076 (1996)]. Here, laboratory measurements in a water waveguide are presented. Taking advantage of the multiple reflections at the interfaces of the guide, high resolution is achieved with the D.O.R.T. method without using any a priori knowledge of the guide. The separation of two scatterers and the selective focusing are obtained with a transverse resolution at least nine times better than the predicted free space limit. The detection of a scatterer placed at less than half a wavelength from the water/air interface is also achieved with good sensitivity. Finally, the impulse response functions of each scatterer to the array is computed as a combination of the eigenvectors of the timereversal operator obtained at each frequency. Using these impulse Green functions, selective focusing with high temporal and spatial compression is performed. 
2pUWb5. A high-resolution algorithm for wave number inversion using a holographic technique. Philippe Roux, Megan McArthur, Paul Hursky, and William A. Kuperman (Marine Physical Lab., Scripps Inst. of Oceanogr., UCSD, 9500 Gilman Dr., La Jolla, CA 92093-0238, philippe@mpl.ucsd.edu)

The aim of this work is to perform a high-resolution wave number inversion in a noisy shallow water waveguide. A single-frequency source is towed at a constant speed relative to a vertical array. No a priori knowledge of the waveguide characteristics is required. The inversion algorithm starts from the holographic representation of the field received from the source on the array. Then, using the recorded field along the source track, a newly modified eigen-matrix pencil was performed to extract the wave numbers. The accuracy of the method is compared to the Cramer-Rao lower bounds. The dependence of the results on the length of the source track, the number of receivers in the array, the signal-to-noise ratio, and the sound velocity profile in the waveguide are discussed. Both experimental and simulated data are reported.

\section{3:40}

2pUWb6. Beamforming performance of a $\mathrm{V}$-shaped array of sea-bed geophone sensors. Richard J. Brind and Nick J. Goddard (DERA Winfrith, Winfrith Technol. Ctr., Dorchester, Dorset DT2 8XJ, UK, rjbrind@dera.gov.uk)

Geophone sensors on the sea-bed offer a mechanism for detecting and localizing acoustic sources in the ocean. Recent work is described in which a V-shaped array of sensor units was deployed on the sea-bed in a shallow water location. Each unit consisted of a three-component set of geophones and a hydrophone. Data were obtained on the response of the sensors to noise from ships in the vicinity, and to impacts on the sea-bed remote from the array. The positions of the units were determined by examining the phase of broadband energy at each pair of elements, and applying a least squares fit. The geophone data were then beamformed. Conventional and adaptive beamforming techniques have been applied, and the sensitivity of each to errors in the knowledge of the element positions assessed. The response of the array to noise from nearby ships has been compared to predictions. The beam pattern was found to be superior to that of an equivalent array of acoustic sensors. The ability of the V-shaped configuration in the experiment to resolve the wave number of the incident field has been investigated. The response of the array to energy arriving in the dispersive interface wave propagation path has been examined.

4:00-4:20 Break
2pUWb7. Transient, tonal, and background noise filtering with wavelet and cosine transforms. Eric Delory and John R. Potter (Acoust. Res. Lab., Tropical Marine Sci. Inst. and Elec. Eng. Dept., Natl. Univ. of Singapore, 27 Engineering Dr., 117596 Singapore, eric@arl.nus.edus.g)

In underwater acoustic signal processing, a major concern has been to extract information from a noisy time series, without over-constraining the estimation. Often, very little is known about the signal or noise and any strict assumption increases the risk of failure in real applications. Here, a new denoising method is presented, tested on three types of real underwater noise sources $\left(n_{1}(t), n_{2}(t), n_{3}(t)\right)$, each added to an arbitrary signal $s(t)$. Noise class indices respectively refer to snapping shrimp noise, shipping noise, and underwater background noise, which are technically considered as transient, tonal, and broadband pink noise. $s(t)$ is defined as any signal that does not belong to these three classes, thus imposing no strict constraint. The extraction process is performed in three steps, each performing a noise-specific orthogonal block transform. First, a one-level Haar wavelet-packet transform is applied to detect transient noise components, using an additive information cost function as a hypothesis test and a simple dot-product to identify and extract them. Second, the denoised signal is reconstructed and decomposed by a one-level cosine-packet transform to identify and extract tonal components in a same manner. After reconstruction, pink noise is removed by thresholding a near-optimal Daubechies wavelet-packet basis.

\section{$4: 40$}

2pUWb8. Objective function particularity for shallow water waveguide parameter estimation procedure. Alexey A. Pudovkin (N. N. Andreev Acoust. Institut, North Brunch, Pochtovay St., 3, Severomorsk, Murmansk reg., 184600, Russia, vfo@nordacin.murmansk.su)

The acoustic field observable parameter values and its acoustic field model predictions were compared, when one desired to estimate model parameters. The measurement was completed when its error was known. It is a preferred tool for analysis to describe statistically disalignments of the model predictions and experimental observations; then one tries to avoid it by creating a sophisticated physic model. Stochastic description acceptability is checked by stochastic hypotheses test (SHT). One tests the hypothesis which was used for objective function (OF) conformation, for instance, by maximum likelihood (ML) tenet. The more disalignment, the bigger OF value for acceptable OF. The model parameters are estimated by OF minimization. The ML OF modification is proposed by penalty function (PF) method. The PF takes into account hypothesis test results, which implicate an acceptable physical-stochastic model of the waveguide acoustic field. If SHT does not give cause to reject the acoustic field formation model on acceptable significance level, then it is possible to calculate the estimation error with the use of ML OF and PF. The measurement implementation main difficulty provided for the model finding. This model ought to be rather simple to calculate the error, but at the same time rather complete to describe disalignments correctly.

\section{Contributed Poster}

This poster will be on display in the Poster Gallery from Monday to Wednesday, 15-17 March. Author will be at the poster from 10:00 a.m. to 12:00 noon on Wednesday, 17 March.

2pUWb9. Matched-field depth discrimination with active sonar. Granger W. Hickman and Jeffrey L. Krolik (Dept. of Elec. and Computer Eng., Box 90291, Duke Univ., Durham, NC 27708)

This paper concerns the problem of estimating target depth in a shallow ocean using multiple pings from a low-frequency active sonar. Although much work on passive range-depth source localization using fullwave propagation models has been published, little attention has been given to the active sonar problem because of the difficulty in modeling target reflection characteristics. In this paper, active MFP is achieved with- out knowledge of the target's backscatter coefficients. By assuming the target's aspect and depth are relatively constant during its track, a MFP range-depth estimate is obtained by modeling the changes in the unresolved coherent multipath returns from ping to ping. The MAP rangedepth estimate involves a dynamic programming procedure which uses a Markov model of changes in the target backscatter coefficients from ping to ping. Preliminary simulation results suggest that the target depth can be resolved (after approximately ten pings) to within $10 \mathrm{~m}$ in a water column $215 \mathrm{~m}$ deep. [Work supported by ONR.] 